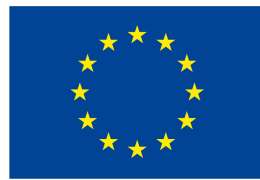

EUROPEAN UNION

European Structural and Investment Funds

Operational Programme Research,

Development and Education

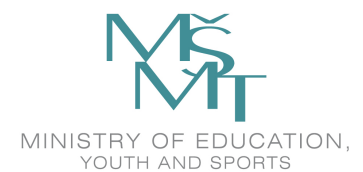

\title{
Workbook for Mathematics II
}

\author{
Jana Volná, Petr Volný
}

\author{
VSB TECHNICAL \\ UNIVERSITY \\ OF OSTRAVA
}




\section{Thanks}

Technology for the Future 2.0, CZ.02.2.69/0.0/0.0/18_058/0010212

ISBN 978-80-248-4510-4

DOI $10.31490 / 9788024845104$ 


\section{Contents}

1 Integral calculus of functions of one real variable $\quad 5$

1.1 Indefinite integrals, antiderivatives . . . . . . . . . . . 5

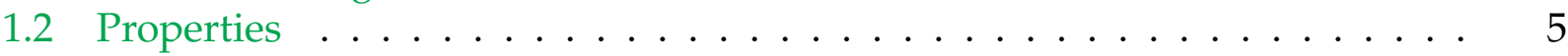

1.3 Elementary integrals . . . . . . . . . . . . . . . . . . . 6

1.4 Direct method of integration . . . . . . . . . . . . . . . . 7

1.5 Integration by parts . . . . . . . . . . . . . . . . 9

1.6 Integration by substitution . . . . . . . . . . . . . . . . . . 11

1.7 Rational functions . . . . . . . . . . . . . . . . . . . 15

1.8 Trigonometric functions . . . . . . . . . . . . . . . . . . 19

1.9 Irrational functions . . . . . . . . . . . . . . . . 22

1.10 Definite integrals, geometric interpretation . . . . . . . . . . . . 23

1.11 Definite integrals, definition . . . . . . . . . . . . . . . . . 24

1.12 Definite integrals, properties . . . . . . . . . . . . . . . . . 25

1.13 Integration by parts and substitution . . . . . . . . . . . . 28

1.14 Definite integrals, rational functions . . . . . . . . . . . . . 30

1.15 Improper integral of the first kind . . . . . . . . . . . . . . . . . . 31

1.16 Improper integral of the second kind . . . . . . . . . . . . . . . . . . . 34

1.17 Geometrical application, area of planar regions . . . . . . . . . . . . . . . . . . . . . . . . . . .

1.18 Length of planar curves . . . . . . . . . . . . . . . . . . . . . 40

1.19 Volume of solids of revolution . . . . . . . . . . . . . . . . . . . . . . . . . . . . . . . . .

1.20 Lateral surface of solids of revolution . . . . . . . . . . . . . . . 46

1.21 Physical applications, mass center and moments . . . . . . . . . . . . 48

1.22 Homogeneous curves . . . . . . . . . . . . . . . . . . . . . . . . . . . . . . . . . . . . . . . .

1.23 Homogeneous regions . . . . . . . . . . . . . . . . . 52

2 Differential calculus of functions of two real variables 55

2.1 Functions of two real variables, domains . . . . . . . . . . . . . 55

2.2 Functions of two real variables, graphs . . . . . . . . . . . . . . 57

2.3 Contour lines . . . . . . . . . . . . . . . . . 58

2.4 Limits, continuity . . . . . . . . . . . . . . . . . . . . . . . . . . . . . . . . . . . 69

2.5 Partial derivatives . . . . . . . . . . . . . . . . . . . . . 62

2.6 Geometrical meaning of partial derivatives . . . . . . . . . . . . . . . 63

2.7 Differentials . . . . . . . . . . . . . . . . . . . 66

2.8 Geometrical meaning of differential, formulas . . . . . . . . . . . . . . 67

2.9 Tangent plane, normal line, Taylor polynomial . . . . . . . . . . . . . . . . 70

2.10 Implicit functions . . . . . . . . . . . . . . . . . . . . . . . . 73

2.11 Local extrema . . . . . . . . . . . . . . . . . . . . . . . . . . . . . . . . . . . .

2.12 Constraint extrema . . . . . . . . . . . . . . . . . . 80

2.13 Global extrema . . . . . . . . . . . . . . . . . . . 84

3 Ordinary differential equations $\quad \mathbf{8 7}$

3.1 Differential equations of the $n$-th order . . . . . . . . . . . . 87

3.2 First order ordinary differential equations . . . . . . . . . . . . . . . 88

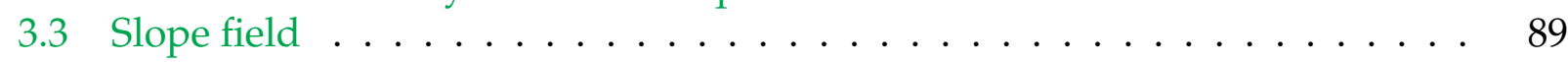

3.4 Cauchy problem . . . . . . . . . . . . . . . . . . . . . 90

3.5 Separable differential equations . . . . . . . . . . . . . . . . . . . . 90 
3.6 Separable ODE of the type $y^{\prime}=f(x) g(y) \ldots \ldots \ldots \ldots \ldots$. . . . . 91

3.7 Separable ODE of the type $y^{\prime}=f(a x+b y+c) \ldots \ldots \ldots \ldots \ldots$

3.8 Separable ODE of the type $y^{\prime}=f\left(\frac{y}{x}\right) \ldots \ldots \ldots \ldots \ldots \ldots \ldots \ldots$

3.9 Linear differential equations of the first order . . . . . . . . . . . . 99

3.10 Linear diff. equations of the second order with constant coefficients . . . . 102

3.11 Characteristic equation . . . . . . . . . . . . . . . . . . . . 103

3.12 Non-homogeneous second order LODE - solution . . . . . . . . . . . . 106

3.13 Variation of constants . . . . . . . . . . . . . . . . . . . . . . . . 107

3.14 Undetermined coefficients . . . . . . . . . . . . . . . . . . . . . . 110 


\section{Integral calculus of functions of one real variable}

\subsection{Indefinite integrals, antiderivatives}

In the calculus of functions depending on one real variable we met very important objects, derivatives of functions. We assigned to every function $f(x)$ the new one, $f^{\prime}(x)$. The preceding task has changed, now we try to find to every function $f(x)$ a function $F(x)$ such that $F^{\prime}(x)=f(x)$. There is a question. Which function do I need to differentiate to get original given function $f(x)$ ?

\section{Definition}

Let a function $f(x)$ is defined on an open interval $I$. The function $F(x)$ is called antiderivative of the function $f(x)$ on $I$ if it holds $F^{\prime}(x)=f(x)$ for every $x \in I$.

\section{Theorem}

Let the function $F(x)$ is antiderivative of $f(x)$ on $I$, then any other antiderivative of the function $f(x)$ on $I$ can be written in the form $F(x)+c$, where constant $c \in \mathbb{R}$.

\section{Remark}

If an antiderivative does exist, there are infinitely many antiderivatives which differ only by a constant $c$. We know that if one construct tangent line at a point $x$ to a given function, the derivative evaluated at this point is slope of this tangent line. The graphs of antiderivatives are displaced parallel in the direction of the $y$-axis. Tangent lines to graphs at the given points $x$ are parallel (they have the same slope), i.e. they have the same derivative.

\subsection{Properties}

\section{Definition}

The set of all antiderivatives of the function $f(x)$ on $I$ is called indefinite integral of the function $f(x)$ an is denoted by $\int f(x) \mathrm{d} x$. Thus,

$$
\int f(x) \mathrm{d} x=F(x)+c, \quad x \in I .
$$

Remark

- The function $f(x)$ is called integrand.

- The expression $\mathrm{d} x$ is differential of $x$, for simplicity, it gives us information about the name of independent variable. The meaning of this object will be discussed later.

- The number $c$ is called constant of integration. 


\section{Properties:}

Theorem

To every function $y=f(x)$ continuous on the interval $I$ there exists indefinite integral $\int f(x) \mathrm{d} x$ which is again a continuous function on $I$.

The following very important theorem represents key properties which are necessary for elementary calculations of indefinite integrals.

Theorem

If there exist two integrals $\int f(x) \mathrm{d} x$ and $\int g(x) \mathrm{d} x$ on $I$, then there also exist the integrals of their sum, difference and multiple of the constant,

$$
\begin{aligned}
\int(f(x) \pm g(x)) \mathrm{d} x & =\int f(x) \mathrm{d} x \pm \int g(x) \mathrm{d} x \\
\int k \cdot f(x) \mathrm{d} x & =k \int f(x) \mathrm{d} x, \quad k \in \mathbb{R} .
\end{aligned}
$$

\subsection{Elementary integrals}

1.

$$
\int 0 \mathrm{~d} x=c
$$

2.

$$
\int x^{n} \mathrm{~d} x=\frac{x^{n+1}}{n+1}+c, \quad n \neq-1, x>0
$$

3.

$$
\int \mathrm{e}^{x} \mathrm{~d} x=\mathrm{e}^{x}+c
$$

4.

$$
\int a^{x} \mathrm{~d} x=\frac{a^{x}}{\ln a}+c, \quad a>0, a \neq 1
$$

5.

$$
\int \frac{1}{x} \mathrm{~d} x=\ln |x|+c, \quad x \neq 0
$$

6.

$$
\int \sin x \mathrm{~d} x=-\cos x+c
$$

7.

$$
\int \cos x d x=\sin x+c
$$

8. $\quad \int \frac{1}{\cos ^{2} x} \mathrm{~d} x=\tan x+c, \quad x \neq(2 k+1) \frac{\pi}{2}$

9. $\quad \int \frac{1}{\sin ^{2} x} \mathrm{~d} x=-\cot x+c, \quad x \neq k \pi$

10. $\int \frac{1}{\sqrt{1-x^{2}}} \mathrm{~d} x=\arcsin x+c, \quad|x|<1$

11. $\int \frac{1}{1+x^{2}} \mathrm{~d} x=\arctan x+c$

12. $\quad \int \frac{f^{\prime}(x)}{f(x)} \mathrm{d} x=\ln |f(x)|+c, \quad f(x) \neq 0$

13. $\int f(a x+b) \mathrm{d} x=\frac{1}{a} F(a x+b)+c, \quad a \neq 0$ 
13a. $\quad \int(a x+b)^{n} \mathrm{~d} x=\frac{1}{a} \frac{(a x+b)^{n+1}}{n+1}+c, \quad a \neq 0$

13b. $\quad \int \mathrm{e}^{a x+b} \mathrm{~d} x=\frac{1}{a} \mathrm{e}^{a x+b}+c, \quad a \neq 0$

13c. $\quad \int \frac{1}{a x+b} \mathrm{~d} x=\frac{1}{a} \ln |a x+b|+c, \quad a \neq 0$

13d. $\int \sin (a x+b) \mathrm{d} x=-\frac{1}{a} \cos (a x+b)+c, \quad a \neq 0$

13e. $\int \cos (a x+b) \mathrm{d} x=\frac{1}{a} \sin (a x+b)+c, \quad a \neq 0$

13f. $\quad \int \frac{1}{\cos ^{2}(a x)} \mathrm{d} x=\frac{1}{a} \tan (a x)+c, \quad a x \neq(2 k+1) \frac{\pi}{2}, a \neq 0$

13g. $\quad \int \frac{1}{\sin ^{2}(a x)} \mathrm{d} x=-\frac{1}{a} \cot (a x)+c, \quad a x \neq k \pi, a \neq 0$

13h. $\int \frac{1}{\sqrt{d^{2}-x^{2}}} \mathrm{~d} x=\arcsin \frac{x}{d}+c, \quad\left|\frac{x}{d}\right|<1, d \neq 0$

13i. $\quad \int \frac{1}{d^{2}+x^{2}} \mathrm{~d} x=\frac{1}{d} \arctan \frac{x}{d}+c, \quad d \neq 0$

\subsection{Direct method of integration}

Example 1

Calculate the integral $\int\left(7 \sqrt[3]{x^{2}}+\frac{1}{2} \sin x-\frac{2}{1+x^{2}}\right) \mathrm{d} x$.

We need to integrate the sum of functions. We get the sum of three integrals,

$$
\int 7 \sqrt[3]{x^{2}} \mathrm{~d} x+\int \frac{1}{2} \sin x \mathrm{~d} x-\int \frac{2}{1+x^{2}} \mathrm{~d} x
$$

Constants can be factored out from the single integrals and the function $\sqrt[3]{x^{2}}$ one can rewrite as $x^{\frac{2}{3}}$,

$$
7 \int x^{\frac{2}{3}} \mathrm{~d} x+\frac{1}{2} \int \sin x \mathrm{~d} x-2 \int \frac{1}{1+x^{2}} \mathrm{~d} x .
$$

Finally, we can find appropriate antiderivatives using formulas 2, 6 and 11 .

Result:

$$
\begin{aligned}
\int\left(7 \sqrt[3]{x^{2}}+\frac{1}{2} \sin x-\frac{2}{1+x^{2}}\right) \mathrm{d} x & =7 \frac{x^{\frac{5}{3}}}{\frac{5}{3}}-\frac{1}{2} \cos x-2 \arctan x+c \\
& =\frac{21}{5} \sqrt[3]{x^{5}}-\frac{1}{2} \cos x-2 \arctan x+c
\end{aligned}
$$




\section{Example 2}

Calculate the integral $\int\left(\frac{\mathrm{e}^{2 x}-1}{\mathrm{e}^{x}+1}+\frac{4}{1-\cos ^{2} x}\right) \mathrm{d} x$.

Let us divide our integral into the sum of two integrals,

$$
\int \frac{\mathrm{e}^{2 x}-1}{\mathrm{e}^{x}+1} \mathrm{~d} x+\int \frac{4}{1-\cos ^{2} x} \mathrm{~d} x
$$

For the integration of the first integral we use the formula $a^{2}-b^{2}=(a-b)(a+b)$, i.e.

$$
\int \frac{\mathrm{e}^{2 x}-1}{\mathrm{e}^{x}+1} \mathrm{~d} x=\int \frac{\left(\mathrm{e}^{x}\right)^{2}-1}{\mathrm{e}^{x}+1} \mathrm{~d} x=\int \frac{\left(\mathrm{e}^{x}-1\right)\left(\mathrm{e}^{x}+1\right)}{\mathrm{e}^{x}+1} \mathrm{~d} x=\int\left(\mathrm{e}^{x}-1\right) \mathrm{d} x=\mathrm{e}^{x}-x+c .
$$

For the second integral using formula 9 and trigonometric formula $\sin ^{2} x+\cos ^{2} x=1$ we get

$$
\int \frac{4}{1-\cos ^{2} x} \mathrm{~d} x=4 \int \frac{1}{\sin ^{2} x} \mathrm{~d} x=-4 \cot x+c .
$$

Result:

$$
\int\left(\frac{\mathrm{e}^{2 x}-1}{\mathrm{e}^{x}+1}+\frac{4}{1-\cos ^{2} x}\right) \mathrm{d} x=\mathrm{e}^{x}-x-4 \cot x+c .
$$

Example 3

Calculate the integrals $\int \mathrm{e}^{-3 x+1} \mathrm{~d} x, \int \frac{1}{\sqrt{4-(3 x-1)^{2}}} \mathrm{~d} x$.

Solution for the integral $\int \mathrm{e}^{-3 x+1} \mathrm{~d} x$.

Integrand is of the form $\mathrm{e}^{a x+b}$, then we use linear substitution, the formula $13 \mathrm{~b}$, where $a=-3, b=1$. We can directly find an antiderivative .

Result:

$$
\int \mathrm{e}^{-3 x+1} \mathrm{~d} x=-\frac{1}{3} \mathrm{e}^{-3 x+1}+c
$$

Solution for the integral $\int \frac{1}{\sqrt{4-(3 x-1)^{2}}} \mathrm{~d} x$.

The integrand is of the form $\frac{1}{\sqrt{d^{2}-(a x+b)^{2}}}$, then we use the formulas 13 and $13 \mathrm{~h}$, where $a=3, b=-1, d=2$.

Result:

$$
\int \frac{1}{\sqrt{4-(3 x-1)^{2}}} \mathrm{~d} x=\frac{1}{3} \arcsin \frac{3 x-1}{2}+c .
$$

Let us note that formulas 13a-13i are direct consequences of the formula 1 . This formula represents linear substitution $a x+b=t$. 
Exercise 4

Solve:
a) $\int\left(x^{5}-2 x+\frac{x^{2}}{3}\right) d x$
c) $\int \frac{d x}{1+\cos 2 x}$
e) $\int \frac{x^{2}-1}{x-1} \mathrm{~d} x$
b) $\int(\sqrt{x}+\sqrt[4]{x}) \mathrm{d} x$
d) $\int \frac{(\sqrt{x}+3)^{2}}{x \sqrt{x}} \mathrm{~d} x$
f) $\int \frac{\sin 2 x}{\sin ^{2} x} d x$

\subsection{Integration by parts}

If we integrate a sum or a difference of functions, we simply need to integrate every summand separately. But this is not true for multiplication of functions,

$$
\int f(x) \cdot g(x) \mathrm{d} x \neq \int f(x) \mathrm{d} x \cdot \int g(x) \mathrm{d} x
$$

There is a natural question, is there any way how to integrate multiplication of functions? Let us recall how to differentiate multiplication of functions,

$$
(u \cdot v)^{\prime}=u^{\prime} \cdot v+u \cdot v^{\prime} \Rightarrow u \cdot v^{\prime}=(u \cdot v)^{\prime}-u^{\prime} \cdot v .
$$

After integration we get

$$
\int u \cdot v^{\prime} \mathrm{d} x=u \cdot v-\int u^{\prime} \cdot v \mathrm{~d} x
$$

\section{Theorem}

Let the functions $u(x)$ and $v(x)$ have continuous derivatives on the interval $I$, then

$$
\int u(x) \cdot v^{\prime}(x) \mathrm{d} x=u(x) \cdot v(x)-\int u^{\prime}(x) \cdot v(x) \mathrm{d} x .
$$

The method is called integration by parts.

Note that the alternative expression

$$
\int u^{\prime}(x) \cdot v(x) \mathrm{d} x=u(x) \cdot v(x)-\int u(x) \cdot v^{\prime}(x) \mathrm{d} x
$$

represents the same formula for integration by parts method.

The point is to consider a multiplication of two functions in such a way that one factor must be differentiated and the second one integrated. The integration is not complete because we replace the original integral by the new one. But the new integral is usually simpler from the point of view of the integration and quite often it can be directly integrated. 


\section{Typical integrals suitable for the integration by parts method}

Let $P(x)$ be a polynomial. The following categories of integrals can be solved by the integration by parts method:

$$
\int P(x) \mathrm{e}^{\alpha x} \mathrm{~d} x, \int P(x) \sin (\alpha x) \mathrm{d} x, \int P(x) \cos (\alpha x) \mathrm{d} x
$$

and

$$
\int P(x) \arctan x \mathrm{~d} x, \int P(x) \ln ^{m} x \mathrm{~d} x \text {. }
$$

The fist group consists of integrals which can be integrated in such a way that for differentiation we choose polynomials, every differentiation of a polynomial lowers its order, so we can repeat the procedure until the polynomial becomes a constant.

For the second group, we have to differentiate the second factors.

Remark

Sometimes, it may happen that after the integration by parts we get an integral which differs from the original integral by some constant factor,

$$
\int f(x) \mathrm{d} x=h(x)+\alpha \int f(x) \mathrm{d} x
$$

where $\alpha \neq 1$. By converting of both integrals to one side of the preceding equation we get

$$
\int f(x) \mathrm{d} x=\frac{1}{1-\alpha} h(x)+c .
$$

\section{Example 5}

Calculate the integral $\int(-2 x+3) \cos 3 x \mathrm{~d} x$.

The integral is of the form $\int P(x) \cos a x \mathrm{~d} x$, thus we use integration by parts method, where the polynomial $P(x)$ is differentiated and the function $\cos a x$ is integrated.

$$
\begin{array}{ll}
u=-2 x+3 & v^{\prime}=\cos 3 x \\
u^{\prime}=-2 & v=\frac{1}{3} \sin 3 x
\end{array}
$$

We get

$\int(-2 x+3) \cos 3 x d x=\frac{-2 x+3}{3} \sin 3 x-\int \frac{-2}{3} \sin 3 x d x=\frac{-2 x+3}{3} \sin 3 x+\frac{2}{3} \int \sin 3 x d x$.

We get simpler integral $\int \sin 3 x \mathrm{~d} x$, which can be directly integrated,

$$
\int \sin 3 x \mathrm{~d} x=-\frac{1}{3} \cos 3 x+c
$$

Result:

$$
\int(-2 x+3) \cos 3 x d x=\frac{-2 x+3}{3} \sin 3 x-\frac{2}{9} \cos 3 x+c .
$$


Example 6

Calculate the integral $\int \ln ^{2} x \mathrm{~d} x$.

The function $\ln ^{2} x$ can not be integrated directly. We can use integration by parts method, but at first we formally have to rewrite the integral in the following way, we need to add the constant factor equal to one: $\int 1 \cdot \ln ^{2} x \mathrm{~d} x$.

The scheme:

$$
\begin{array}{ll}
u=\ln ^{2} x & v^{\prime}=1 \\
u^{\prime}=2 \frac{\ln x}{x} & v=x
\end{array}
$$

We get

$$
\int \ln ^{2} x \mathrm{~d} x=x \ln ^{2} x-2 \int x \frac{\ln x}{x} \mathrm{~d} x=x \ln ^{2} x-2 \int \ln x \mathrm{~d} x
$$

The new integral $\int \ln x \mathrm{~d} x$ can also be integrated using integration by parts method, we need to repeat the preceding steps,

$$
\begin{array}{ll}
u=\ln x & v^{\prime}=1 \\
u^{\prime}=\frac{1}{x} & v=x
\end{array}
$$

We get

$$
\int \ln x \mathrm{~d} x=x \ln x-\int x \frac{1}{x} \mathrm{~d} x=x \ln x-\int 1 \mathrm{~d} x=x \ln x-x+c
$$

Result:

Exercise 7

$$
\int \ln ^{2} x \mathrm{~d} x=x \ln ^{2} x-2(x \ln x-x)+c
$$

Solve:
a) $\int\left(x^{2}-3\right) \sin 2 x d x$
c) $\int \frac{\ln x}{x^{2}} \mathrm{~d} x$
e) $\int \frac{x}{\sin ^{2} x} d x$
b) $\int x \mathrm{e}^{2 x} \mathrm{~d} x$
d) $\int(2 x-1) \ln x \mathrm{~d} x$
f) $\int \mathrm{e}^{x} \sin x \mathrm{~d} x$

\subsection{Integration by substitution}

Substitution is the most important method for integration of functions. It has the same meaning as integration by parts method. Our task is to simplify an integral as much as possible to get directly integrable function.

Substitution of the type $\varphi(x)=t$

Theorem

Let the function $f(t)$ has an antiderivative $F(t)$ on an open interval $J$, the function $\varphi(x)$ is differentiable on an open interval $I$ and for arbitrary $x \in I$ holds $\varphi(x) \in J$. Then the 
function $F(\varphi(x))$ is the antiderivative of the function $f(\varphi(x)) \varphi^{\prime}(x)$ on $I$ and holds

$$
\int f(\varphi(x)) \varphi^{\prime}(x) \mathrm{d} x=\int f(t) \mathrm{d} t=F(t)+c=F(\varphi(x))+c .
$$

From the previous theorem we see how the integrand must look in order to use the substitution method. It must be an expression that is composed of the product of a composite function and a derivative of its interior component. The issue is that such product is not always visible at the first sight, thus the integrand must be appropriately modified.

Algorithm:

- we choose the substitution $\varphi(x)=t$

- the preceding equality must be differentiated $\varphi^{\prime}(x) \mathrm{d} x=\mathrm{d} t$

- in the integral $\int f(\varphi(x)) \varphi^{\prime}(x) \mathrm{d} x$ we replace $\varphi(x)$ by a new variable $t$ and the expression $\varphi^{\prime}(x) \mathrm{d} x$ by $\mathrm{d} t$

- we integrate $\int f(t) \mathrm{d} t$ with respect to the new variable $t$

- we return to the original variable $x$

Linear substitution: $a x+b=t$

If the function $f(t)$ has an antiderivative $F(t)$, i.e. $\int f(t) \mathrm{d} t=F(t)+c$, then the following relation is satisfied,

$$
\int f(a x+b) \mathrm{d} x=\frac{1}{a} F(a x+b)+c, \quad a, b \in \mathbb{R}, \quad a \neq 0 .
$$

Formulas derived from the above formula can be found on the page 7.

Example 8

Calculate the integral $\int x \tan \left(x^{2}-2\right) \mathrm{d} x$.

For substitution we choose interior component of the composite function $\tan \left(x^{2}-2\right)$,

$$
\begin{aligned}
x^{2}-2 & =t \\
2 x \mathrm{~d} x & =\mathrm{d} t \\
x \mathrm{~d} x & =\frac{1}{2} \mathrm{~d} t
\end{aligned}
$$

We get

$$
\int x \tan \left(x^{2}-2\right) \mathrm{d} x=\int \tan \left(x^{2}-2\right) \cdot x \mathrm{~d} x=\int(\tan t) \cdot \frac{1}{2} \mathrm{~d} t=\frac{1}{2} \int \tan t \mathrm{~d} t .
$$

We obtained the integral $\int \tan t \mathrm{~d} t$ which can be rewritten as $\int \frac{\sin t}{\cos t} \mathrm{~d} t$. 
Notice that we see a derivative of denominator in numerator (up to a constant multiple), thus the following formula can be used,

$$
\int \frac{f^{\prime}(x)}{f(x)} \mathrm{d} x=\ln |f(x)|+c \Rightarrow \int \frac{\sin t}{\cos t} \mathrm{~d} t=-\int \frac{-\sin t}{\cos t} \mathrm{~d} t=-\ln |\cos t|+c .
$$

We return to the original variable.

Result:

Exercise 9

$$
\int x \tan \left(x^{2}-2\right) \mathrm{d} x=-\frac{1}{2} \ln \left|\cos \left(x^{2}-2\right)\right|+c .
$$

Solve:
a) $\int \frac{\mathrm{d} x}{\left(x^{2}+1\right) \sqrt{\arctan x}}$
c) $\int \frac{(3+\ln x)^{5}}{x} \mathrm{~d} x$
b) $\int \mathrm{e}^{x} \cos \left(\mathrm{e}^{x}\right) \mathrm{d} x$
d) $\int \frac{\tan x}{\cos ^{2} x} \mathrm{~d} x$

\section{Substitution of the type $x=\varphi(t)$}

From the preceding substitution category follows that the integral $\int f(\varphi(x)) \varphi^{\prime}(x) \mathrm{d} x$ can be rewritten by means of the substitution $\varphi(x)=t$ on the integral with a new variable $\int f(t) \mathrm{d} t$. Sometimes, it is necessary to choose a different approach and replace the original variable by a new function. Consider that we have to calculate the integral $\int f(x) \mathrm{d} x$. By means of the substitution $x=\varphi(t)$ and $\mathrm{d} x=\varphi^{\prime}(t) \mathrm{d} t$ we try to replace the integral by a new integral $\int f(\varphi(t)) \varphi^{\prime}(t) \mathrm{d} t$. To find an antiderivative, the following must be satisfied:

- $f(x)$ is continuous on $(a, b)$

- $x=\varphi(t)$ is strictly monotonic on $(\alpha, \beta)$ and $\varphi^{\prime}(t) \neq 0$ is continuous on $(\alpha, \beta)$.

If both assumptions are fulfilled, then the inverse function $\varphi^{-1}(x) \neq 0$ exists and $t=$ $\varphi^{-1}(x)$.

\section{- Theorem}

Let a function $f(x)$ be continuous on an interval $J$, let a strictly monotonic function $\varphi(t)$ have a derivative on $I$ not equal to zero for every $t \in I$ and let it hold $\varphi(I)=J$. Then $f(x)$ has an antiderivative $F\left(\varphi^{-1}(x)\right)$ on $J$ and it holds

$$
\int f(x) \mathrm{d} x=\int f(\varphi(t)) \varphi^{\prime}(t) \mathrm{d} t=F\left(\varphi^{-1}(x)\right)+c .
$$


By means of the substitution method we usually integrate irrational functions.

- Integrand contains the expression $\sqrt[n]{a x+b}$. We choose the following substitutions $a x+b=t^{n}$ and $\mathrm{d} x=n t^{n-1} \mathrm{~d} t$.

- If the integrand consists of irrational functions with different roots $\sqrt[n_{1}]{a x+b}, \sqrt[n_{2}]{a x+b}$, ..., we choose the substitution $a x+b=t^{n}$, where $n$ is the least common multiple of the numbers $n_{1}, n_{2}, \ldots$

- Integrand contains the expression $\sqrt{a^{2}-b^{2} x^{2}}$. The substitution is called trigonometric because we set $b x=a \sin t$ or $b x=a \cos t$, i.e. $\mathrm{d} x=\frac{a}{b} \cos t \mathrm{~d} t$ or $\mathrm{d} x=-\frac{a}{b} \sin t \mathrm{~d} t$.

Example 10

Calculate the integral $\int \sin \sqrt{2 x+3} \mathrm{~d} x$.

At first we need to cancel the root,

$$
\begin{aligned}
2 x+3 & =t^{2} \\
x & =\frac{t^{2}-3}{2} \\
\mathrm{~d} x & =t \mathrm{~d} t
\end{aligned}
$$

We get

$$
\int \sin \sqrt{2 x+3} \mathrm{~d} x=\int t \sin \sqrt{t^{2}} \mathrm{~d} t=\int t \sin t \mathrm{~d} t
$$

New integral is of the form $\int P(t) \sin a t \mathrm{~d} t$, which can be solved by the integration by parts method.

$$
\begin{array}{rlrl}
u & =t & v^{\prime} & =\sin t \\
u^{\prime} & =1 & v & =-\cos t
\end{array}
$$

We obtain

$$
\int t \sin t \mathrm{~d} t=-t \cos t+\int \cos t \mathrm{~d} t=-t \cos t+\sin t+c .
$$

We return to the original variable $t=\sqrt{2 x+3}$.

Result:

$$
\int \sin \sqrt{2 x+3} \mathrm{~d} x=-\sqrt{2 x+3} \cos \sqrt{2 x+3}+\sin \sqrt{2 x+3}+c .
$$

\section{Exercise 11}

Solve:
a) $\int \frac{\mathrm{d} x}{(2+x) \sqrt{1+x}}$
c) $\int \mathrm{e}^{\sqrt{x}} \mathrm{~d} x$
b) $\int \frac{\cot \sqrt{x}}{\sqrt{x}} \mathrm{~d} x$
d) $\int \arctan x d x$ 


\subsection{Rational functions}

Every rational function of the form $f(x)=\frac{P(x)}{Q(x)}$, where $P(x)$ and $Q(x)$ are polynomials of arbitrary degrees can be expressed as

$$
\frac{P(x)}{Q(x)}=S(x)+R_{1}(x)+\ldots+R_{s}(x),
$$

where $S(x)$ is a polynomial and $R_{1}(x), \ldots, R_{S}(x)$ are partial fractions.

Partial fractions are special rational functions. We specify two types:

$$
\frac{A}{(x-\alpha)^{k}}, \quad k \in \mathbb{N} ; \alpha, A \in \mathbb{R},
$$

where the polynomial $x-\alpha$ has real $\operatorname{root} \alpha \in \mathbb{R}$ and

$$
\frac{M x+N}{\left(x^{2}+p x+q\right)^{k}}=\frac{B(2 x+p)+C}{\left(x^{2}+p x+q\right)^{k}}, k \in \mathbb{N} ; B, C, M, N, p, q \in \mathbb{R} ; p^{2}-4 q<0,
$$

where the polynomial $x^{2}+p x+q$ has two complex conjugate roots.

Definition

Rational function $\frac{P(x)}{Q(x)}$ is called proper, if $\operatorname{deg} P(x)<\operatorname{deg} Q(x)$, where $\operatorname{deg} P(x)$ is the degree of the polynomial $P(x)$.

\section{Partial fraction decomposition of proper rational functions}

- find the roots of the polynomial in denominator

- make decomposition

- multiply the equation by the polynomial in denominator

- find the coefficient of decomposition by either comparative method or substitution method or combination of both methods.

Example 12

Calculate the integral $\int \frac{x^{4}+2}{x-1} \mathrm{~d} x$.

The polynomial in numerator has degree equal to four, the polynomial in denominator has degree equal to one. The function is not proper, we need to divide polynomials using standard Euclidean algorithm. 


$$
\begin{aligned}
& \left(x^{4}+2\right):(x-1)=x^{3}+x^{2}+x+1+\frac{3}{x-1} \\
& \frac{-\left(x^{4}-x^{3}\right)}{x^{3}+2} \\
& \frac{-\left(x^{3}-x^{2}\right)}{x^{2}+2} \\
& \frac{-\left(x^{2}-x\right)}{x+2} \\
& \frac{-(x-1)}{3}
\end{aligned}
$$

We get

$$
\int \frac{x^{4}+2}{x-1} \mathrm{~d} x=\int\left(x^{3}+x^{2}+x+1+\frac{3}{x-1}\right) \mathrm{d} x
$$

Result:

$$
\int \frac{x^{4}+2}{x-1} \mathrm{~d} x=\frac{x^{4}}{4}+\frac{x^{3}}{3}+\frac{x^{2}}{2}+x+3 \ln |x-1|+c
$$

\section{Exercise 13}

Express the rational function $R(x)=\frac{x^{3}+2}{x-1}$ as a sum of a polynomial and a proper rational function.

\section{Example 14}

Make partial fraction decomposition of the function $R(x)=\frac{x^{3}+6 x^{2}+3 x+2}{x^{4}+x^{3}+x^{2}}$.

We check that the given function is proper rational function. Indeed, the degree of the polynomial in numerator is equal to 3 , the degree of the polynomial in denominator is equal to 4. Thus, the given rational function is proper, $3<4$.

Let us make partial fraction decomposition of the given proper rational function,

$$
R(x)=\frac{x^{3}+6 x^{2}+3 x+2}{x^{4}+x^{3}+x^{2}}=\frac{x^{3}+6 x^{2}+3 x+2}{x^{2}\left(x^{2}+x+1\right)}=\frac{A}{x}+\frac{B}{x^{2}}+\frac{C(2 x+1)+D}{x^{2}+x+1} .
$$

Note that it is better to use alternative expression of a partial fraction of the second type from the point of view of subsequent integration.

We need to multiply the above equality by polynomial in denominator to find values of constants $A, B, C$ and $D$,

$$
x^{3}+6 x^{2}+3 x+2=A x\left(x^{2}+x+1\right)+B\left(x^{2}+x+1\right)+(C(2 x+1)+D) x^{2} .
$$


We use comparative method to find the values. We compare coefficients of both sides of the above equation.

$$
\begin{aligned}
x^{3}+6 x^{2}+3 x+2=(A+2 C) x^{3}+(A+B & C+D) x^{2}+(A+B) x+B \\
x^{0}: 2=B & \Rightarrow B=2 \\
x^{1}: 3=A+B & \Rightarrow A=1 \\
x^{3}: 1=A+2 C & \Rightarrow C=0 \\
x^{2}: 6=A+B+C+D & \Rightarrow D=3
\end{aligned}
$$

Result:

Exercise 15

$$
R(x)=\frac{1}{x}+\frac{2}{x^{2}}+\frac{3}{x^{2}+x+1}
$$

Make partial fraction decomposition:
a) $R(x)=\frac{1}{x^{3}-4 x^{2}+4 x}$
b) $R(x)=\frac{x}{(x-1)\left(x^{2}+1\right)}$
c) $R(x)=\frac{2 x-1}{x^{3}-4 x}$

\section{Integration of partial fractions with real roots in denominator}

For simple roots:

$$
\int \frac{A}{x-\alpha} \mathrm{d} x=A \ln |x-\alpha|+c
$$

For $k$-fold roots $(k>1)$ :

$$
\int \frac{A}{(x-\alpha)^{k}} \mathrm{~d} x=\frac{A}{(1-k)(x-\alpha)^{k-1}}+c .
$$

\section{Integration of partial fractions with complex roots in denominator}

The integration of the fraction $\frac{B(2 x+p)}{x^{2}+p x+q}$ leads to:

$$
\int \frac{B(2 x+p)}{x^{2}+p x+q} \mathrm{~d} x=B \ln \left|x^{2}+p x+q\right|+c .
$$

The integration of the fraction $\frac{C}{x^{2}+p x+q}$ must be accompanied by conversion of the triple $x^{2}+p x+q$ onto the perfect square:

$$
\int \frac{C}{x^{2}+p x+q} \mathrm{~d} x=C \int \frac{\mathrm{d} x}{(x+p / 2)^{2}+r^{2}}=\frac{C}{r} \arctan \frac{x+p / 2}{r}+c,
$$

where

$$
r=\sqrt{q-\frac{p^{2}}{4}}
$$


Example 16

Calculate the integral $\int \frac{x+2}{x^{2}+x-6} \mathrm{~d} x$.

The function is proper. We decompose denominator onto root factors,

$$
x^{2}+x-6=(x-2)(x+3)
$$

Partial fraction decomposition:

$$
\frac{x+2}{(x-2)(x+3)}=\frac{A}{x-2}+\frac{B}{x+3} .
$$

Multiplying by $(x-2)(x+3)$,

$$
x+2=A(x+3)+B(x-2) .
$$

Substitution method:

$$
\begin{aligned}
& x=2: \quad 4=5 A \quad \Rightarrow \quad A=\frac{4}{5} \\
& x=-3:-1=-5 B \Rightarrow B=\frac{1}{5}
\end{aligned}
$$

We obtain two partial fractions of the first type,

$$
\int \frac{x+2}{x^{2}+x-6} \mathrm{~d} x=\frac{4}{5} \int \frac{1}{x-2} \mathrm{~d} x+\frac{1}{5} \int \frac{1}{x+3} \mathrm{~d} x
$$

Result:

$$
\int \frac{x+2}{x^{2}+x-6} \mathrm{~d} x=\frac{4}{5} \ln |x-2|+\frac{1}{5} \ln |x+3|+c
$$

\section{Example 17}

Calculate the integral $\int \frac{-3 x+1}{x^{2}+4 x+4} \mathrm{~d} x$.

The function is proper. We decompose denominator onto root factors,

$$
x^{2}+4 x+4=(x+2)^{2} \text {. }
$$

Partial fraction decomposition:

$$
\frac{-3 x+1}{(x+2)^{2}}=\frac{A}{x+2}+\frac{B}{(x+2)^{2}} .
$$

Multiplying by $(x+2)^{2}$,

$$
-3 x+1=A(x+2)+B .
$$

Combination of both methods:

$$
\begin{aligned}
x=-2: 7 & =B \\
x^{0}: 1 & =2 A+B \Rightarrow-6=2 A \Rightarrow A=-3
\end{aligned}
$$

We obtain partial fraction of the first and second type,

$$
\int \frac{-3 x+1}{x^{2}+4 x+4} \mathrm{~d} x=-3 \int \frac{1}{x+2} \mathrm{~d} x+7 \int \frac{1}{(x+2)^{2}} \mathrm{~d} x
$$

Result:

$$
\int \frac{-3 x+1}{x^{2}+4 x+4} \mathrm{~d} x=-3 \ln |x+2|-\frac{7}{x+2}+c .
$$


Example 18

Calculate the integral $\int \frac{x}{x^{2}+3 x+4} \mathrm{~d} x$.

The function is proper. We decompose denominator onto root factors, but the polynomial has no real roots.

Partial fraction decomposition:

$$
\frac{x}{x^{2}+3 x+4}=\frac{B(2 x+3)}{x^{2}+3 x+4}+\frac{C}{x^{2}+3 x+4} .
$$

Multiplying by $\left(x^{2}+3 x+4\right)$,

$$
x=B(2 x+3)+C .
$$

Comparative method:

$$
\begin{aligned}
& x^{1}: 1=2 B \quad \Rightarrow \quad B=\frac{1}{2} \\
& x^{0}: 0=3 B+C \quad \Rightarrow \quad C=-\frac{3}{2}
\end{aligned}
$$

We obtain partial fraction of the second type,

$$
\int \frac{x}{x^{2}+3 x+4} \mathrm{~d} x=\frac{1}{2} \int \frac{2 x+3}{x^{2}+3 x+4} \mathrm{~d} x-\frac{3}{2} \int \frac{1}{x^{2}+3 x+4} \mathrm{~d} x .
$$

We rewrite denominator of the second integrand as $\left(x+\frac{3}{2}\right)^{2}+\frac{7}{4}$ using completing the square method.

Result:

$$
\int \frac{x}{x^{2}+3 x+4} \mathrm{~d} x=\frac{\ln \left(x^{2}+3 x+4\right)}{2}-\frac{3}{\sqrt{7}} \arctan \frac{2\left(x+\frac{3}{2}\right)}{\sqrt{7}}+c .
$$

Exercise 19

Calculate the integrals
a) $\int \frac{x+2}{x^{3}-2 x^{2}-8 x} \mathrm{~d} x$
c) $\int \frac{x^{3}}{x^{3}+x} \mathrm{~d} x$
b) $\int \frac{3 x}{\left(x^{2}+1\right)\left(x^{2}+4\right)} d x$
d) $\int \frac{2 x^{2}-3 x+5}{x^{3}(x+1)} \mathrm{d} x$

\subsection{Trigonometric functions}

Integrals of the type $\int \sin ^{m} x \cos ^{n} x \mathrm{~d} x$, where $m, n \in \mathbb{Z}$

1. If at least one of the numbers $m, n$ is odd, we use the substitution:

$$
\begin{aligned}
& \sin x=t, \text { if } n \text { is odd, } \\
& \cos x=t, \text { if } m \text { is odd. }
\end{aligned}
$$

If both numbers are odd one can choose any of the preceding substitutions. 
2. If both numbers $m, n$ are even and nonnegative, we use formulas for double angle:

$$
\begin{aligned}
& \sin ^{2} x=\frac{1-\cos 2 x}{2}, \\
& \cos ^{2} x=\frac{1+\cos 2 x}{2} .
\end{aligned}
$$

3. If both numbers $m, n$ are even and at least one of them negative, we use the substitution $\tan x=t, x \in\left(-\frac{\pi}{2}, \frac{\pi}{2}\right)$. Then

$$
\begin{aligned}
& \sin x=\frac{t}{\sqrt{1+t^{2}}}, \quad \cos x=\frac{1}{\sqrt{1+t^{2}}} \\
& x=\arctan t \quad \Rightarrow \quad \mathrm{d} x=\frac{1}{1+t^{2}} \mathrm{~d} t .
\end{aligned}
$$

\section{Example 20}

Calculate the integral $\int \cos ^{2} x \sin ^{5} x \mathrm{~d} x$.

The integrand is of the type $\int \sin ^{m} x \cos ^{n} x \mathrm{~d} x$, where cosine has even power $(n=2)$ and sine has odd power $(m=5)$, thus we use the substitution:

$$
\begin{aligned}
\cos x & =t \\
-\sin x \mathrm{~d} x & =\mathrm{d} t
\end{aligned}
$$

We need to manipulate the integrand in the following way:

$$
\int \cos ^{2} x \sin ^{4} x \sin x d x=\int \cos ^{2} x\left(1-\cos ^{2} x\right)^{2} \sin x d x .
$$

after substitution we get:

$$
-\int t^{2}\left(1-t^{2}\right)^{2} \mathrm{~d} t=-\int\left(t^{2}-2 t^{4}+t^{6}\right) \mathrm{d} t=-\frac{t^{3}}{3}+2 \frac{t^{5}}{5}-\frac{t^{7}}{7}+c .
$$

We return to the original variable.

Result:

$$
\int \cos ^{2} x \sin ^{5} x \mathrm{~d} x=-\frac{\cos ^{3} x}{3}+2 \frac{\cos ^{5} x}{5}-\frac{\cos ^{7} x}{7}+c .
$$

Example 21

Calculate the integral $\int \frac{\sin ^{4} x}{\cos ^{8} x} \mathrm{~d} x$.

Integrand is of the type $\int \sin ^{m} x \cos ^{n} x \mathrm{~d} x$, both powers are even and at least one of them is negative, we use the substitution,

$$
\begin{gathered}
\tan x=t \\
\frac{1}{\cos ^{2} x} \mathrm{~d} x=\mathrm{d} t
\end{gathered}
$$


We rewrite the integrand:

$$
\begin{aligned}
\int \frac{\sin ^{4} x}{\cos ^{8} x} \mathrm{~d} x & =\int \tan ^{4} x \frac{1}{\cos ^{2} x} \frac{1}{\cos ^{2} x} \mathrm{~d} x=\int \tan ^{4} x \frac{\sin ^{2} x+\cos ^{2} x}{\cos ^{2} x} \frac{1}{\cos ^{2} x} \mathrm{~d} x \\
& =\int \tan ^{4} x\left(\tan ^{2} x+1\right) \frac{1}{\cos ^{2} x} \mathrm{~d} x .
\end{aligned}
$$

After substitution we get

$$
\int t^{4}\left(t^{2}+1\right) \mathrm{d} t=\frac{t^{7}}{7}+\frac{t^{5}}{5}+c .
$$

We return to the original variable.

Result:

$$
\int \frac{\sin ^{4} x}{\cos ^{8} x} \mathrm{~d} x=\frac{\tan ^{7} x}{7}+\frac{\tan ^{5} x}{5}+c .
$$

Example 22

Calculate the integral $\int \sin ^{2} x \cos ^{2} x \mathrm{~d} x$.

Integrand is of the type $\int \sin ^{m} x \cos ^{n} x \mathrm{~d} x$, both powers are even and nonnegative, then we use the formulas for double angle:

$$
\int \sin ^{2} x \cos ^{2} x \mathrm{~d} x=\int\left(\frac{1-\cos 2 x}{2}\right)\left(\frac{1+\cos 2 x}{2}\right) \mathrm{d} x=\frac{1}{4} \int\left(1-\cos ^{2} 2 x\right) \mathrm{d} x .
$$

Again we use the same formula for double angle:

$$
\frac{1}{4} \int\left(1-\cos ^{2} 2 x\right) \mathrm{d} x=\frac{1}{4} \int\left(1-\frac{1+\cos 4 x}{2}\right) \mathrm{d} x=\frac{1}{4} \int\left(\frac{1}{2}-\frac{\cos 4 x}{2}\right) \mathrm{d} x .
$$

Result:

$$
\int \sin ^{2} x \cos ^{2} x \mathrm{~d} x=\frac{x}{8}-\frac{\sin 4 x}{32}+c
$$

Exercise 23

Calculate the integrals
a) $\int \sin ^{2} x \cos ^{3} x \mathrm{~d} x$
b) $\int \frac{\sin ^{2} x}{\cos ^{6} x} d x$
c) $\int \cos ^{4} x \mathrm{~d} x$

\section{Universal substitution}

$$
\begin{aligned}
\tan \frac{x}{2} & =t, \quad x \in(-\pi, \pi) \\
x & =2 \arctan t \\
\mathrm{~d} x & =\frac{2}{1+t^{2}} \mathrm{~d} t \\
\sin x & =\frac{2 t}{1+t^{2}}, \quad \cos x=\frac{1-t^{2}}{1+t^{2}}
\end{aligned}
$$

Universal substitution is suitable for integrals of the type

$$
\int R(\sin x, \cos x) \mathrm{d} x
$$

where $R(u, v)$ is a rational function in variables $u=\sin x, v=\cos x$. 
Example 24

Calculate the integral $\int \frac{-3}{2+\cos x} \mathrm{~d} x$.

Integrand is a rational function containing trigonometric functions, we use universal substitution:

$$
\int \frac{-3}{2+\cos x} \mathrm{~d} x=\int \frac{-3}{2+\frac{1-t^{2}}{1+t^{2}}} \frac{2}{1+t^{2}} \mathrm{~d} t=\int \frac{-6}{3+t^{2}} \mathrm{~d} t
$$

Using general formula

$$
\int \frac{1}{a^{2}+x^{2}} \mathrm{~d} x=\frac{1}{a} \arctan \frac{x}{a}+c
$$

we get:

$$
\int \frac{-6}{3+t^{2}} \mathrm{~d} t=\frac{-6}{\sqrt{3}} \arctan \frac{t}{\sqrt{3}}+c
$$

Result:

$$
\int \frac{-3}{2+\cos x} \mathrm{~d} x=-2 \sqrt{3} \arctan \frac{\sqrt{3} \tan \frac{x}{2}}{3}+c .
$$

Exercise 25

Calculate the integrals
a) $\int \frac{1}{2 \sin x+1} d x$
b) $\int \frac{1}{1+\cos x+\sin x} \mathrm{~d} x$

\subsection{Irrational functions}

Example 26

Calculate the integral $\int \frac{3}{\sqrt[3]{2 x-1}+2} \mathrm{~d} x$.

Integrand contains the expression $\sqrt[n]{a x+b}$, we the use substitution:

$$
\begin{aligned}
2 x-1 & =t^{3} \\
\mathrm{~d} x & =\frac{3}{2} t^{2} \mathrm{~d} t
\end{aligned}
$$

We get:

$$
\int \frac{3}{\sqrt[3]{2 x-1}+2} \mathrm{~d} x=\int \frac{9 t^{2}}{2(t+2)} \mathrm{d} t .
$$

We integrate an improper rational function, thus we have to divide:

$$
\int \frac{9 t^{2}}{2(t+2)} \mathrm{d} t=\frac{9}{2} \int\left(t-2+\frac{4}{t+2}\right) \mathrm{d} t=\frac{9}{2}\left(\frac{t^{2}}{2}-2 t+4 \ln |t+2|\right)+c .
$$

Result:

$$
\int \frac{3}{\sqrt[3]{2 x-1}+2} \mathrm{~d} x=\frac{9}{2}\left(\frac{\sqrt[3]{(2 x-1)^{2}}}{2}-2 \sqrt[3]{2 x-1}+4 \ln |\sqrt[3]{2 x-1}+2|\right)+c
$$




\section{Example 27}

Calculate the integral $\int \sqrt{9-16 x^{2}} \mathrm{~d} x$.

Integrand contains $\sqrt{a^{2}-b^{2} x^{2}}$, we use the substitution:

$$
\begin{aligned}
4 x & =3 \sin t \\
4 \mathrm{~d} x & =3 \cos t \mathrm{~d} t
\end{aligned}
$$

We get:

$\int \sqrt{9-16 x^{2}} \mathrm{~d} x=\frac{3}{4} \int \sqrt{9-9 \sin ^{2} t} \cos t \mathrm{~d} t=\frac{3}{4} \int \sqrt{9\left(1-\sin ^{2} t\right)} \cos t \mathrm{~d} t=\frac{3}{4} \int 3 \cos ^{2} t \mathrm{~d} t$.

We need to use the formula for double angle:

$$
\begin{aligned}
\frac{9}{4} \int \cos ^{2} t \mathrm{~d} t & =\frac{9}{8} \int(1+\cos 2 t) \mathrm{d} t=\frac{9}{8}\left(t+\frac{\sin 2 t}{2}\right)+c=\frac{9}{8}\left(t+\frac{2 \sin t \cos t}{2}\right)+c \\
& =\frac{9}{8}\left(t+\sin t \sqrt{\left(1-\sin ^{2} t\right)}\right)+c .
\end{aligned}
$$

Result:

$$
\int \sqrt{9-16 x^{2}} \mathrm{~d} x=\frac{9}{8}\left(\arcsin \frac{4 x}{3}+\frac{4 x}{3} \sqrt{1-\frac{16 x^{2}}{9}}\right)+c=\frac{9}{8} \arcsin \frac{4 x}{3}+\frac{x}{2} \sqrt{9-16 x^{2}}+c .
$$

Exercise 28

Calculate the integrals
a) $\int \frac{1+5 x}{\sqrt[3]{x+5}} \mathrm{~d} x$
b) $\int \frac{\sqrt[3]{x}}{x+\sqrt{x}} \mathrm{~d} x$
c) $\int \sqrt{4-x^{2}} \mathrm{~d} x$

\subsection{Definite integrals, geometric interpretation}

Let us consider a nonnegative bounded funtion $f(x)$ continuous on the interval $[a, b]$. It can be proved that a definite integral $\int_{a}^{b} f(x) \mathrm{d} x$ represents area of an planar region surrounded by the graph of $f(x), x$-axis and the lines $x=a, x=b$.

For general function $f(x)$ we are not able to find out area $P$ yet. Let us try to suggest how to calculate such area approximately:

1. We divide given planar region by lines parallel with $y$-axis onto smaller parts. It is clear that area is given by the sum of areas of such smaller parts, simply by adding them together. Denote area of a planar region by $P$. It holds: $P=P_{1}+P_{2}+\ldots+P_{n}$. 
2. We need to find out areas of single parts. These parts are bounded above by the function $f(x)$, thus we make an approximation. We replace these parts by rectangles. We choose a representative of every single divided interval $\xi_{i}$ and in these points we calculate function values. We use these function values to create rectangles and simply calculate their areas:

$$
P \doteq\left(x_{1}-a\right) f\left(\xi_{1}\right)+\left(x_{2}-x_{1}\right) f\left(\xi_{2}\right)+\ldots+\left(b-x_{n-1}\right) f\left(\xi_{n}\right) .
$$

3. It is clear that the final value is not precisely area of the given planar region. The difference between area and its approximation is an error. If we consider more dividing points, the error will be smaller. Area $P$ will be obtained for infinitely many dividing points.

\subsection{Definite integrals, definition}

Definition

If there exists a $\operatorname{limit}\left(\lim _{n \rightarrow \infty} \sum_{i=1}^{n} P_{i}=I\right)$, then we call this limit Riemann integral or Riemann definite integral or definite integral of the function $f(x)$ along the interval $[a, b]$. We write

$$
I=\int_{a}^{b} f(x) \mathrm{d} x
$$

where the number $a$ is called lower limit, the number $b$ is called upper limit and the function $f(x)$ is called integrand.

\section{Remark}

If a function $f(x)$ is continuous on $[a, b]$, then its Riemann integral does exist. After generalization we get the following definition.

\section{Definition}

Let a function $f(x)$ is bounded and piecewise continuous on $[a, b]$, then $f(x)$ has Riemann integral on $[a, b]$.

\section{Calculation of definite integrals}

For calculation of definite integrals we use Newton-Leibniz formula, which represents relation between antiderivatives and definite integrals. 


\section{Definition}

Let $F(x)$ is an antiderivative of the function $f(x)$ on the interval $I$. Then for the numbers $a, b$ belonging to $I$ we define Newton definite integral of the function $f(x)$ with limits $a$ and $b$ by the formula:

$$
\int_{a}^{b} f(x) \mathrm{d} x=[F(x)]_{a}^{b}=F(b)-F(a) .
$$

Remark

Newton integrable functions are also Riemann integrable. Not every Riemann integrable function is Newton integrable.

\subsection{Definite integrals, properties}

Theorem

Let functions $f(x)$ and $g(x)$ be integrable on $[a, b]$, then their sum, difference and constant factor are integrable on this interval and holds:

$$
\begin{gathered}
\int_{a}^{b}(f(x) \pm g(x)) \mathrm{d} x=\int_{a}^{b} f(x) \mathrm{d} x \pm \int_{a}^{b} g(x) \mathrm{d} x \\
\int_{a}^{b} k \cdot f(x) \mathrm{d} x=k \int_{a}^{b} f(x) \mathrm{d} x, c \in \mathbb{R} .
\end{gathered}
$$

Additional properties:

\section{Theorem}

Let $f(x)$ and $g(x)$ be integrable on $[a, b]$, then it holds:

$$
\begin{aligned}
& \int_{a}^{a} f(x) \mathrm{d} x=0 \\
& \int_{b}^{a} f(x) \mathrm{d} x=-\int_{a}^{b} f(x) \mathrm{d} x \\
& \left|\int_{a}^{b} f(x) \mathrm{d} x\right| \leq \int_{a}^{b}|f(x) \mathrm{d} x| \\
& \text { if } f(x) \leq g(x) \forall x \in[a, b] \text {, then } \int_{a}^{b} f(x) \mathrm{d} x \leq \int_{a}^{b} g(x) \mathrm{d} x \text {. }
\end{aligned}
$$


The following property is suitable especially in the cases when integrand does not have a uniform analytic formula on the interval $[a, b]$.

Theorem

Let $f(x)$ be integrable on $[a, b]$ and let $c$ be an arbitrary real number $a<c<b$. Then $f(x)$ is integrable on the intervals $[a, c]$ and $[c, b]$ and holds:

$$
\int_{a}^{b} f(x) \mathrm{d} x=\int_{a}^{c} f(x) \mathrm{d} x+\int_{c}^{b} f(x) \mathrm{d} x
$$

\section{Even and odd functions}

If a function $f(x)$ is even on the interval $[-a, a]$, then

$$
\int_{-a}^{a} f(x) \mathrm{d} x=2 \int_{0}^{a} f(x) \mathrm{d} x
$$

If a function $f(x)$ is odd on the interval $[-a, a]$, then

$$
\int_{-a}^{a} f(x) \mathrm{d} x=0
$$

Example 29

Calculate the integral $\int_{0}^{\pi}\left((4-x)^{2}+\cos 2 x\right) \mathrm{d} x$.

Using properties one can represent the integral as a sum of four integrals:

$$
\int_{0}^{\pi}\left(16-8 x+x^{2}+\cos 2 x\right) \mathrm{d} x=16 \int_{0}^{\pi} \mathrm{d} x-8 \int_{0}^{\pi} x \mathrm{~d} x+\int_{0}^{\pi} x^{2} \mathrm{~d} x+\int_{0}^{\pi} \cos 2 x \mathrm{~d} x .
$$

All integrals are elementary, thus it is easy to find appropriate antiderivatives. Finally, Newton-Leibniz formula is applied:

$$
\begin{gathered}
16 \int_{0}^{\pi} \mathrm{d} x-8 \int_{0}^{\pi} x \mathrm{~d} x+\int_{0}^{\pi} x^{2} \mathrm{~d} x+\int_{0}^{\pi} \cos 2 x \mathrm{~d} x=16[x]_{0}^{\pi}-4\left[x^{2}\right]_{0}^{\pi}+\left[\frac{x^{3}}{3}\right]_{0}^{\pi}+\left[\frac{\sin 2 x}{2}\right]_{0}^{\pi} \\
\quad=16(\pi-0)-4\left(\pi^{2}-0\right)+\left(\frac{\pi^{3}}{3}-0\right)+(0-0)=16 \pi-4 \pi^{2}+\frac{\pi^{3}}{3} .
\end{gathered}
$$

Result:

$$
\int_{0}^{\pi}\left((4-x)^{2}+\cos 2 x\right) d x=16 \pi-4 \pi^{2}+\frac{\pi^{3}}{3} .
$$


Exercise 30

Solve:
a) $\int_{1}^{2}\left(3 x^{2}+1\right) d x$
b) $\int_{0}^{1}\left(3-x^{2}\right)^{2} \mathrm{~d} x$
$\int_{-1}^{1} \frac{x^{2}}{1+x^{2}} \mathrm{~d} x$

Example 31

Calculate the integrals: $\int_{-\frac{\pi}{4}}^{\frac{\pi}{4}} \tan x \mathrm{~d} x, \int_{-1}^{1} \frac{x^{4}}{2} \mathrm{~d} x$.

First integral, $\int_{-\frac{\pi}{4}}^{\frac{\pi}{4}} \tan x \mathrm{~d} x$ :

The function tangent is odd on the interval $\left[-\frac{\pi}{4}, \frac{\pi}{4}\right]$, thus the integral is null.

Verify:

$$
\int_{-\frac{\pi}{4}}^{\frac{\pi}{4}} \tan x \mathrm{~d} x=\int_{-\frac{\pi}{4}}^{\frac{\pi}{4}} \frac{\sin x}{\cos x} \mathrm{~d} x=-[\ln |\cos x|]_{-\frac{\pi}{4}}^{\frac{\pi}{4}}=-\left(\ln \frac{\sqrt{2}}{2}-\ln \frac{\sqrt{2}}{2}\right)=0 .
$$

Second integral, $\int_{-1}^{1} \frac{x^{4}}{2} \mathrm{~d} x$ :

The function $\frac{x^{4}}{2}$ is even on the interval $[-1,1]$, thus:

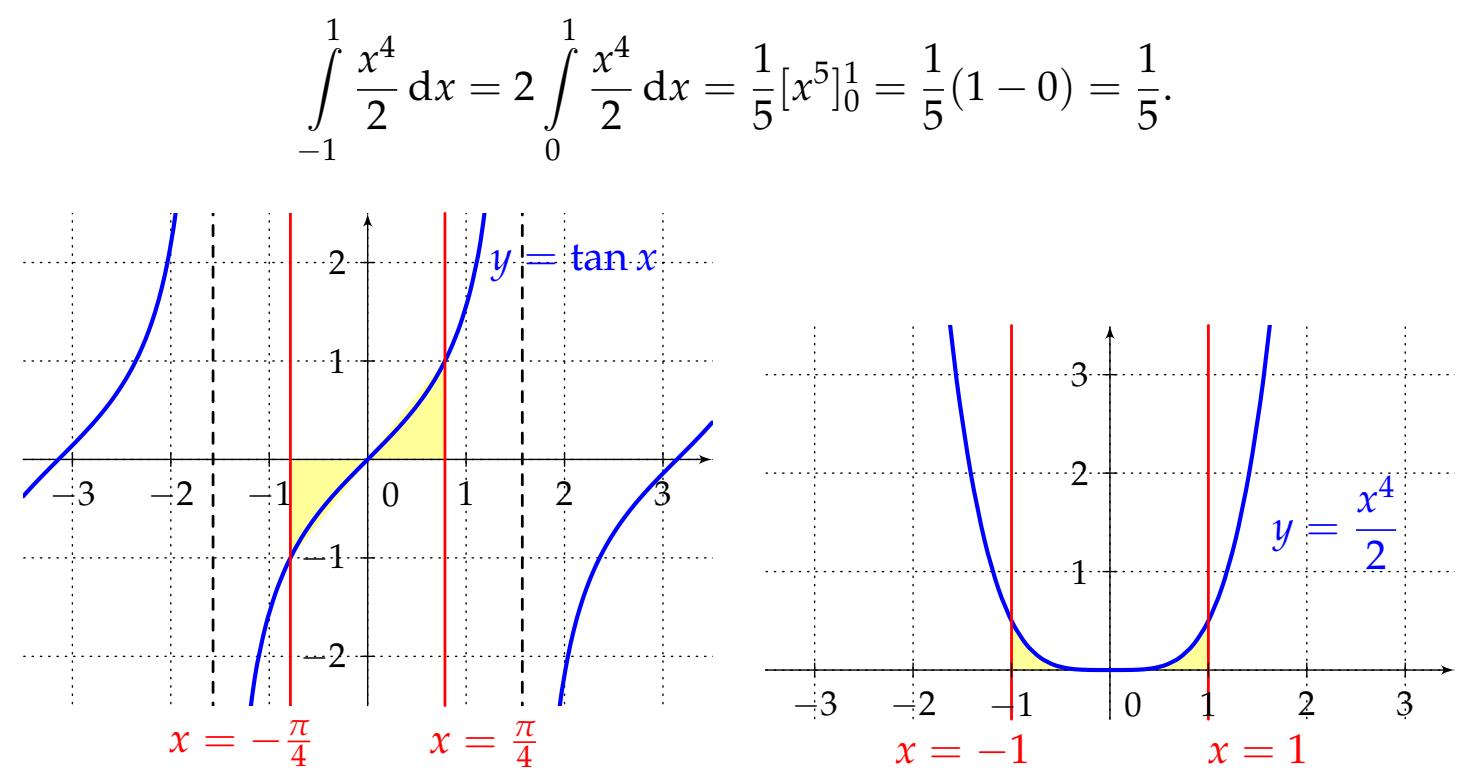


Exercise 32

Solve:
a) $\int_{-\frac{\pi}{4}}^{\frac{\pi}{4}}\left(x^{2}+\cos x\right) d x$
b) $\int_{-1}^{1} \frac{5 x}{2 x^{2}+1} \mathrm{~d} x$
c) $\int_{-2}^{2} 2 \mathrm{~d} x$

\subsection{Integration by parts and substitution}

\section{Integration by parts for definite integrals}

Theorem

Let functions $u(x)$ and $v(x)$ have derivatives integrable on $[a, b], a<b$, then it holds

$$
\int_{a}^{b} u(x) \cdot v^{\prime}(x) \mathrm{d} x=[u(x) \cdot v(x)]_{a}^{b}-\int_{a}^{b} u^{\prime}(x) \cdot v(x) \mathrm{d} x .
$$

\section{Remark}

We use this method in the same way as in the case of indefinite integrals. There is an advantage, we can evaluate antiderivatives due to ongoing calculation. The calculation gets shorter and smoother.

\section{Substitution for definite integrals}

\section{Theorem}

If a function $f(x)$ is integrable on $[a, b]$ and strictly monotonic function $x=\varphi(t)$ has continuous derivative $\varphi^{\prime}(t)$ on the interval $[\alpha, \beta]$, whereas $\varphi(\alpha)=a$ and $\varphi(\beta)=b$, then it holds:

$$
\int_{a}^{b} f(x) \mathrm{d} x=\int_{\alpha}^{\beta} f(\varphi(t)) \varphi^{\prime}(t) \mathrm{d} t
$$

\section{Remark}

The calculation procedure and the notation are similar as in the case of indefinite integrals, only new limits must be determined. There is an advantage, we do not need to return to the original variable after substitution.

Example 33

Calculate the integral $\int_{1}^{2} \ln x^{2} \mathrm{~d} x$. 
Integrand is a composite function, let us try the integration by parts method:

$$
\begin{aligned}
u & =\ln x^{2} & v^{\prime} & =1 \\
u^{\prime} & =\frac{2}{x} & v & =x
\end{aligned}
$$

After the application:

$$
\int_{1}^{2} \ln x^{2} \mathrm{~d} x=\left[x \ln x^{2}\right]_{1}^{2}-\int_{1}^{2} 2 \mathrm{~d} x=2 \ln 4-\ln 1-2[x]_{1}^{2}=2 \ln 4-2(2-1) .
$$

Result:

$$
\int_{1}^{2} \ln x^{2} \mathrm{~d} x=2(\ln 4-1)
$$

Exercise 34

Solve:
a) $\int_{0}^{1}(x+2) \mathrm{e}^{x} \mathrm{~d} x$
b) $\int_{0}^{\sqrt{3}} x \arctan x \mathrm{~d} x$

Example 35

Calculate the integral $\int_{0}^{\pi} x \cos x^{2} \mathrm{~d} x$.

Integrand is in the form of a multiple of two functions, where derivative of interior function is directly the other function of the multiple (it differs up to a constant), we use the substitution:

$$
\begin{gathered}
x^{2}=t \\
2 x \mathrm{~d} x=\mathrm{d} t
\end{gathered}
$$

New limits for $t$ :

$$
\begin{array}{ll}
\text { lower limit : } & 0 \mapsto 0^{2}=0 \\
\text { upper limit : } & \pi \mapsto \pi^{2}
\end{array}
$$

After the application:

$$
\int_{0}^{\pi} x \cos x^{2} \mathrm{~d} x=\frac{1}{2} \int_{0}^{\pi^{2}} \cos t \mathrm{~d} t=\frac{1}{2}[\sin t]_{0}^{\pi^{2}}=\frac{1}{2}\left(\sin \pi^{2}-0\right) .
$$

Result:

$$
\int_{0}^{\pi} x \cos x^{2} \mathrm{~d} x=\frac{\sin \pi^{2}}{2}
$$


Example 36

Calculate the integral $\int_{3}^{8} \frac{x}{\sqrt{x+1}+1} \mathrm{~d} x$.

It is integral with the root. We use the substitution:

$$
\begin{aligned}
x+1 & =t^{2} \\
\mathrm{~d} x & =2 t \mathrm{~d} t
\end{aligned}
$$

New limits for $t$ :

lower limit : $\quad 3 \mapsto \sqrt{3+1}=2$

upper limit : $\quad 8 \mapsto \sqrt{8+1}=3$

After the application:

$\int_{3}^{8} \frac{x}{\sqrt{x+1}-1} \mathrm{~d} x=\int_{2}^{3} 2 t \frac{t^{2}-1}{t+1} \mathrm{~d} t=2 \int_{2}^{3} t(t-1) \mathrm{d} t=2\left[\frac{t^{3}}{3}-\frac{t^{2}}{2}\right]_{2}^{3}=2\left(\frac{27}{3}-\frac{9}{2}-\left(\frac{8}{3}-\frac{4}{2}\right)\right)$.

Result:

Exercise 37

$$
\int_{3}^{8} \frac{x}{\sqrt{x+1}-1} \mathrm{~d} x=\frac{23}{3}
$$

Solve:
a) $\int_{1}^{\mathrm{e}} \frac{1+\ln x}{x} \mathrm{~d} x$
c) $\int_{1}^{3} x \ln \left(x^{2}+2\right) \mathrm{d} x$
b) $\int_{0}^{\frac{\pi}{2}} \sin x \sqrt{\cos x} d x$
d) $\int_{0}^{\frac{1}{2}} \arcsin 2 x d x$

\subsection{Definite integrals, rational functions}

Example 38

Calculate the integral $\int_{1}^{3} \frac{7 x+6}{x^{2}\left(x^{2}+x+6\right)} \mathrm{d} x$.

The function is proper, we can make partial fraction decomposition. Denominator has two complex conjugate roots and one double root equal to 0 .

Partial fraction decomposition: $\frac{7 x+6}{x^{2}\left(x^{2}+x+6\right)}=\frac{A}{x}+\frac{B}{x^{2}}+\frac{C(2 x+1)}{x^{2}+x+6}+\frac{D}{x^{2}+x+6}$ 
Multiplying $x^{2}\left(x^{2}+x+6\right)$

$$
7 x+6=A x\left(x^{2}+x+6\right)+B\left(x^{2}+x+6\right)+C(2 x+1) x^{2}+D x^{2}
$$

Comparative method:

$$
\begin{gathered}
x^{3}: 0=A+2 C \quad \Rightarrow C=-\frac{1}{2} A \Rightarrow C=-\frac{1}{2} \\
x^{2}: 0=A+B+C+D \Rightarrow D=-\frac{3}{2} \\
x^{1}: 7=6 A+B \quad \Rightarrow \quad \Rightarrow=1 \\
x^{0}: 6=6 B \quad 3=1 \\
\int_{1}^{3} \frac{7 x+6}{x^{2}\left(x^{2}+x+6\right)} \mathrm{d} x=\int_{1}^{3} \frac{1}{x} \mathrm{~d} x+\int_{1}^{3} \frac{1}{x^{2}} \mathrm{~d} x-\frac{1}{2} \int_{1}^{3} \frac{2 x+1}{x^{2}+x+6} \mathrm{~d} x-\frac{3}{2} \int_{1}^{3} \frac{1}{x^{2}+x+6} \mathrm{~d} x \\
=[\ln |x|]_{1}^{3}-\left[\frac{1}{x}\right]_{1}^{3}-\frac{1}{2}\left[\ln \left(x^{2}+x+6\right)\right]_{1}^{3}-\frac{6}{\sqrt{23}}\left[\arctan \frac{2\left(x+\frac{1}{2}\right)}{\sqrt{23}}\right]_{1}^{3} \\
=\ln 3-\frac{1}{3}+1-\frac{1}{2}(\ln 16-\ln 8)-\frac{6 \sqrt{23}}{23}\left(\arctan \frac{7 \sqrt{23}}{23}-\arctan \frac{3 \sqrt{23}}{23}\right) .
\end{gathered}
$$

Exercise 39

Solve:
a) $\int_{1}^{\sqrt{3}} \frac{x+2}{x\left(x^{2}+1\right)} d x$
b) $\int_{1}^{2} \frac{x-1}{x^{3}(x+1)} d x$

\subsection{Improper integral of the first kind}

\section{Definition}

Let a function $f(x)$ be continuous on the interval $[a, \infty)$, then the integral

$$
\int_{a}^{\infty} f(x) \mathrm{d} x=\lim _{c \rightarrow \infty} \int_{a}^{c} f(x) \mathrm{d} x=L
$$

is called improper integral of the first kind. If $L \in \mathbb{R}$, we say that the improper integral is convergent. In the opposite case $(L=\infty$ or $L=-\infty)$ we say that the improper integral is divergent. 
- Remark

- Quite analogously we define improper integral of the first kind on the interval $(-\infty, a]$.

- We integrate a bounded function on an unbounded interval.

- If a function $f(x)$ is continuous on the interval $(-\infty, \infty)$ and both improper integrals are convergent for arbitrary number $a$,

$$
L_{1}=\int_{-\infty}^{a} f(x) \mathrm{d} x, \quad L_{2}=\int_{a}^{\infty} f(x) \mathrm{d} x
$$

then

$$
\int_{-\infty}^{\infty} f(x) \mathrm{d} x=L_{1}+L_{2}
$$

Example 40

Calculate the integrals: $\int_{0}^{\infty} \frac{1}{1+x^{2}} \mathrm{~d} x, \quad \int_{-\infty}^{-1} \frac{1}{x} \mathrm{~d} x, \int_{-\infty}^{\infty} \frac{1}{1+x^{2}} \mathrm{~d} x$.

First integral:

Given function is bounded on the interval $[0, \infty)$, thus it is improper integral of the first kind:

$$
\int_{0}^{\infty} \frac{1}{1+x^{2}} \mathrm{~d} x=\lim _{c \rightarrow \infty} \int_{0}^{c} \frac{1}{1+x^{2}} \mathrm{~d} x=\lim _{c \rightarrow \infty}\left([\arctan ]_{0}^{c}\right)=\lim _{c \rightarrow \infty} \arctan c=\frac{\pi}{2} .
$$

Improper integral is convergent.

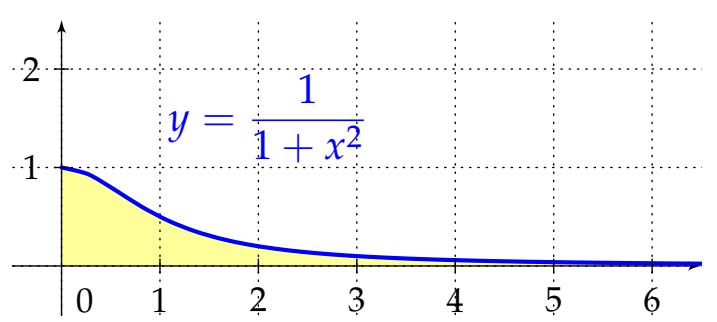

Second integral:

Given function is bounded on the interval $(-\infty,-1]$, thus it is improper integral of the first kind:

$$
\begin{aligned}
\int_{-\infty}^{-1} \frac{1}{x} \mathrm{~d} x & =\lim _{c \rightarrow-\infty} \int_{c}^{-1} \frac{1}{x} \mathrm{~d} x=\lim _{c \rightarrow-\infty}\left([\ln |x|]_{c}^{-1}\right) \\
& =\lim _{c \rightarrow-\infty}\left([\ln (-x)]_{c}^{-1}\right)=\lim _{c \rightarrow-\infty}(-\ln (-c))=-\infty
\end{aligned}
$$

Improper integral is divergent. 


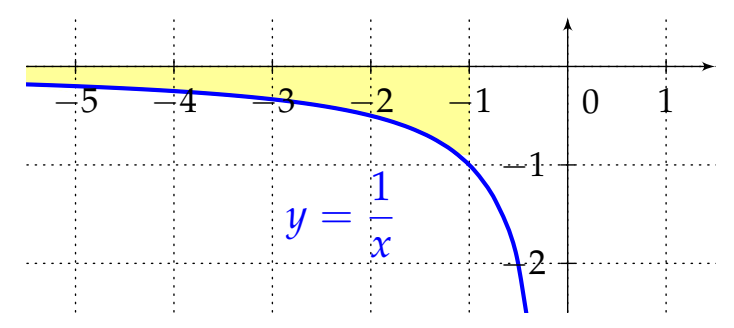

Third integral:

$$
\begin{aligned}
\int_{-\infty}^{\infty} \frac{1}{1+x^{2}} \mathrm{~d} x & =\int_{-\infty}^{0} \frac{1}{1+x^{2}} \mathrm{~d} x+\int_{0}^{\infty} \frac{1}{1+x^{2}} \mathrm{~d} x \\
& =\lim _{c \rightarrow-\infty} \int_{c}^{0} \frac{1}{1+x^{2}} \mathrm{~d} x+\lim _{c \rightarrow \infty} \int_{0}^{c} \frac{1}{1+x^{2}} \mathrm{~d} x=\frac{\pi}{2}+\frac{\pi}{2}=\pi .
\end{aligned}
$$

Integration domain $(-\infty, \infty)$ we divide appropriately into two parts. Note that the function is even, thus the improper integral on the left part of the interval has the same value as the improper integral on the right part of the interval.

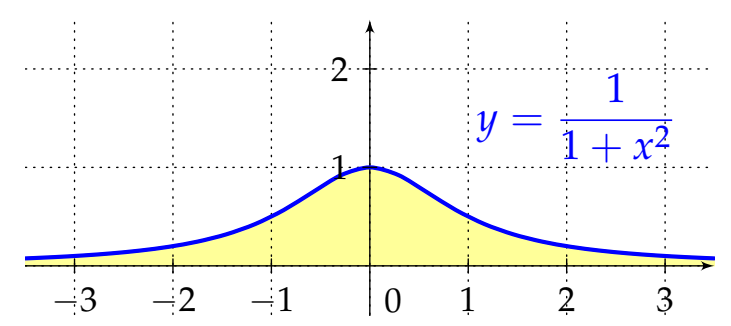

\section{Exercise 41}

Solve:

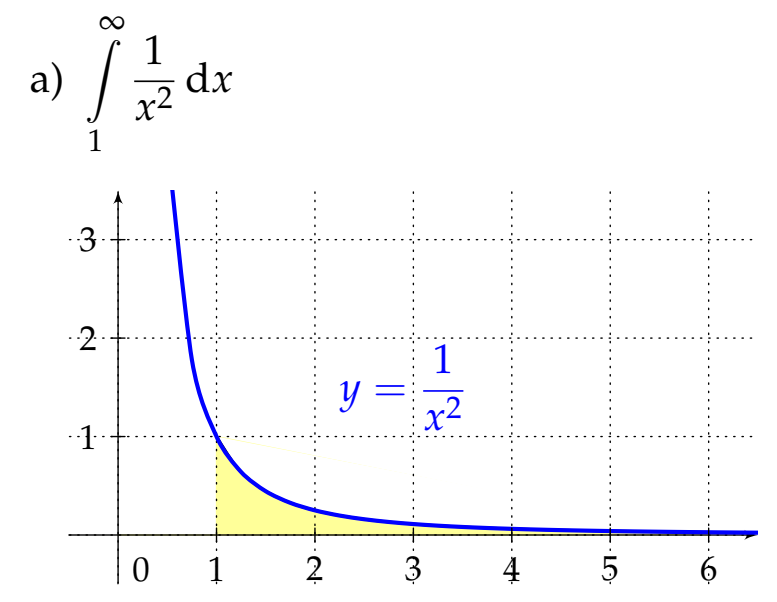

b) $\int_{-\infty}^{-1} \frac{\mathrm{d} x}{x^{2}+2 x+5}$

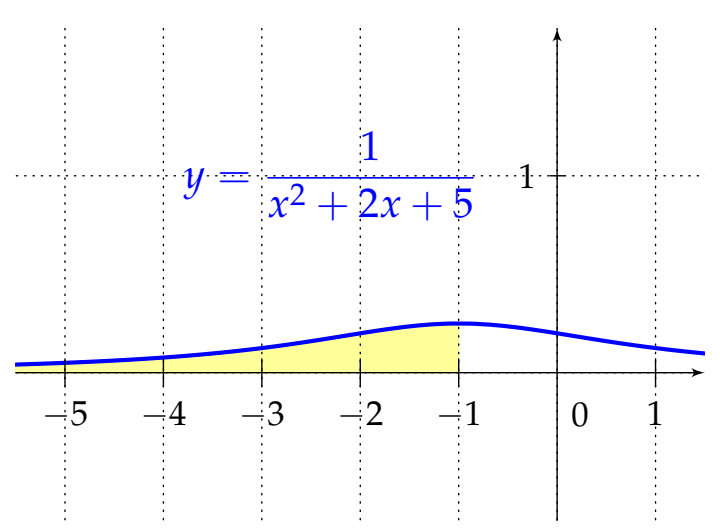




\subsection{Improper integral of the second kind}

\section{Definition}

Let a function $f(x)$ be continuous and unbounded on the interval $[a, b)$, then the integral

$$
\int_{a}^{b} f(x) \mathrm{d} x=\lim _{c \rightarrow b^{-}} \int_{a}^{c} f(x) \mathrm{d} x=L
$$

is called improper integral of the second kind. If $L \in \mathbb{R}$, we say that the improper integral is convergent. In the opposite case $(L=\infty$ or $L=-\infty)$ we say that the improper integral is divergent.

Remark

- Quite analogously we define improper integral of the second kind on the interval $(a, b]$.

- We integrate unbounded function on bounded interval.

- If a function $f(x)$ has more points in which it is unbounded, we divide the interval into corresponding number of subintervals such that every single subinterval contains only one such point. If improper integrals are convergent on the subintervals, the integral is convergent on the entire interval, we get it as a sum of partial values of single integrals. To check divergence of the improper integral, it is enough that at least one of single integrals is divergent.

Example 42

Calculate the integral: $\int_{0}^{1} \frac{x}{\sqrt{1-x^{2}}} \mathrm{~d} x$.

The function is unbounded on the interval $[0,1)$, it is improper integral of the second kind:

$$
\int_{0}^{1} \frac{x}{\sqrt{1-x^{2}}} \mathrm{~d} x=\lim _{c \rightarrow 1^{-}} \int_{0}^{c} \frac{x}{\sqrt{1-x^{2}}} \mathrm{~d} x
$$

Substitution:

$$
\begin{gathered}
1-x^{2}=t \\
-2 x \mathrm{~d} x=\mathrm{d} t
\end{gathered}
$$

New limits for $t$ :

lower limit : $\quad 0 \mapsto 1$

upper limit : $\quad c \mapsto 1-c^{2}$

After the application:

$$
\begin{aligned}
\int_{0}^{1} \frac{x}{\sqrt{1-x^{2}}} \mathrm{~d} x & =\lim _{c \rightarrow 1^{-}} \int_{0}^{c} \frac{x}{\sqrt{1-x^{2}}} \mathrm{~d} x=\lim _{c \rightarrow 1^{-}}\left(-\frac{1}{2} \int_{0}^{1-c^{2}} \frac{\mathrm{d} t}{\sqrt{t}}\right)=\lim _{c \rightarrow 1^{-}}[-\sqrt{t}]_{1}^{1-c^{2}} \\
& =\lim _{c \rightarrow 1^{-}}\left(-\sqrt{1-c^{2}}+1\right)=1
\end{aligned}
$$


Improper integral is convergent.

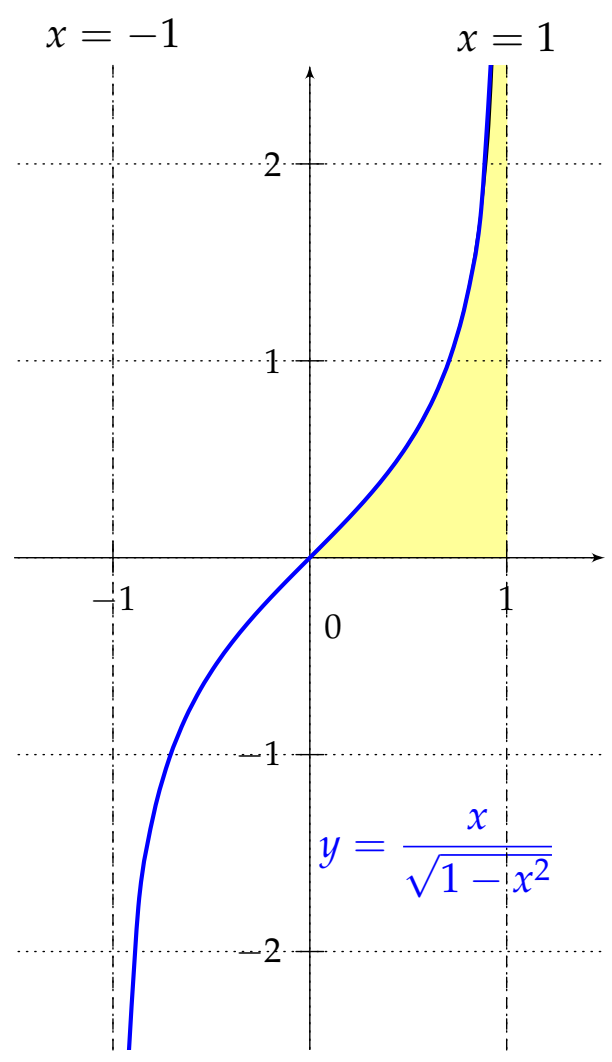

Exercise 43

Solve:
a) $\int_{0}^{\frac{\pi}{4}} \frac{d x}{\sin x \cos x}$
b) $\int_{0}^{1} \ln x \mathrm{~d} x$
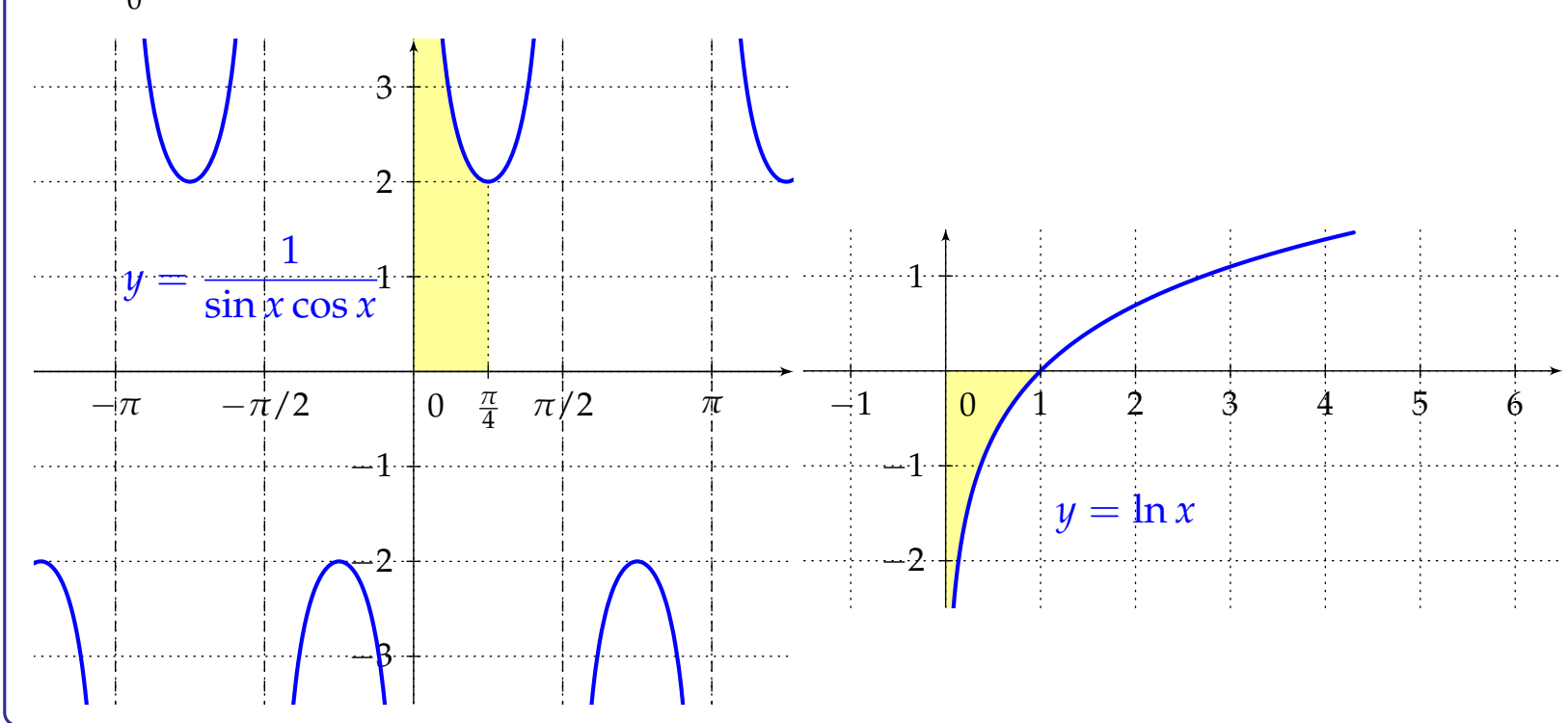


\subsection{Geometrical application, area of planar regions}

If a planar region is surrounded by $x$-axis, the lines $x=a, x=b$ and the graph of a continuous non-negative function $y=f(x)$, then area of such planar region is determined by a definite integral, it is simply geometrical interpretation of definite integrals:

$$
P=\int_{a}^{b} f(x) \mathrm{d} x
$$

In the case that a function $y=f(x)$ is negative on the interval $[a, b]$, the definite integral is also negative. Due to the fact that area must be positive for arbitrary planar region, we need to add absolute value to guarantee positivity of the result:

$$
P=\int_{a}^{b}|f(x)| \mathrm{d} x=-\int_{a}^{b} f(x) \mathrm{d} x .
$$

If a function $y=f(x)$ is either non-negative or non-positive somewhere on the interval $[a, b]$, then we need to divide the interval on single subintervals on which the function is only non-negative or non-positive. Then we calculate appropriate areas of such single planar regions. Stress that negative results must become positive.

If a planar region is surrounded by graphs of two functions $y=f(x)$ and $y=g(x)$, $f(x) \geq g(x)$ on the interval $[a, b]$, and the lines $x=a, x=b$, its area is given by:

$$
P=\int_{a}^{b}(f(x)-g(x)) \mathrm{d} x .
$$

In the case that we do not know integration domain, we have to find out $x$ coordinates of intersection points of both graphs, thus we have to solve the equation $f(x)=g(x)$.

If a function $f$ is represented by parametric equations $x=\varphi(t), y=\psi(t), t \in[\alpha, \beta]$, where the function $\psi(t)$ is continuous and non-negative on $[\alpha, \beta]$ and the function $\varphi(t)$ has on the interval $[\alpha, \beta]$ derivative $\dot{\varphi}(t)$ different from zero and $\dot{\varphi}(t)$ is integrable on $[\alpha, \beta]$, it holds for area of a planar region surrounded by the graph of such function $f$ on the interval $[\alpha, \beta]$ :

$$
P=\left|\int_{\alpha}^{\beta} \psi(t) \dot{\varphi}(t) \mathrm{d} t\right| .
$$

Example 44

Sketch the graph of the function $y=-x^{3}+5 x+2$ and calculate area of a planar region surrounded by this graph, $x$-axis and the lines
a) $x=1, x=2$,
b) $x=-2, x=-1$. 
We sketch the region whose area we are looking for:

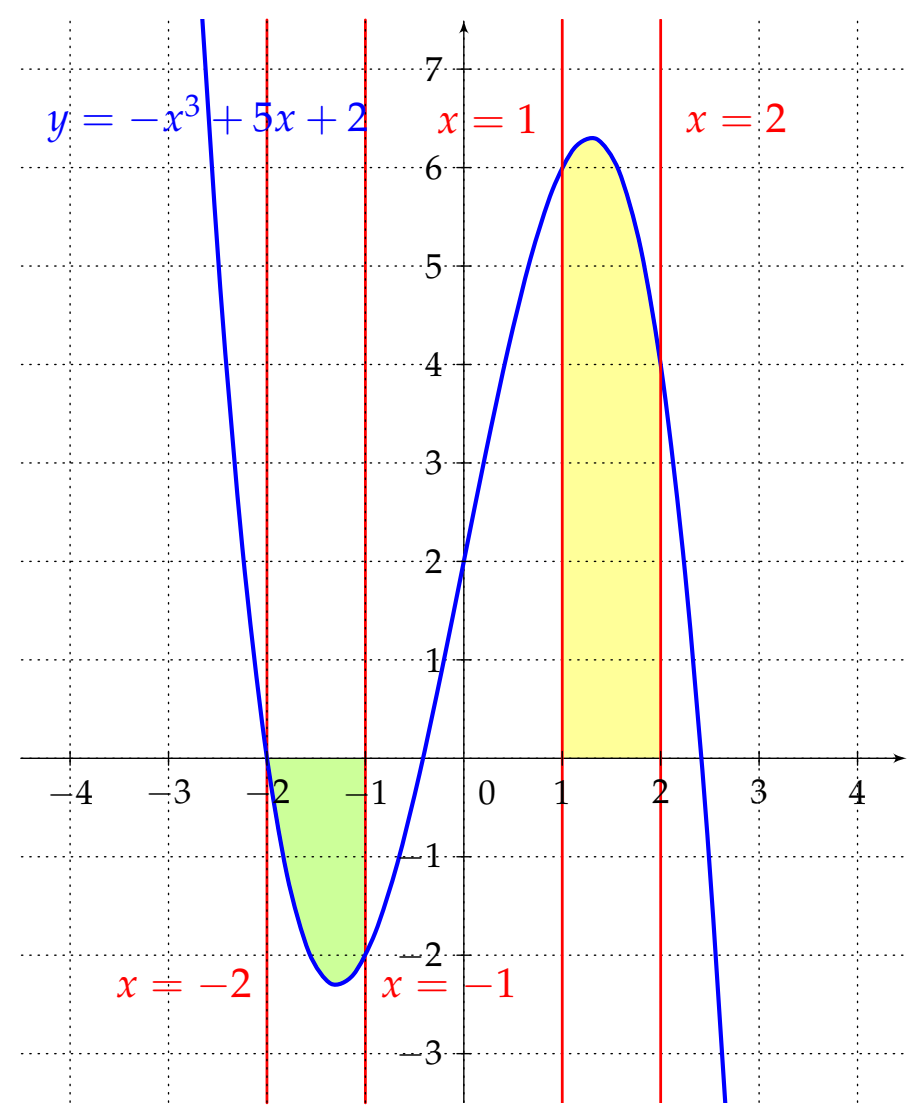

a)

We see that the function is non-negative on the interval $[1,2]$ (yellow area):

$$
P=\int_{1}^{2}\left(-x^{3}+5 x+2\right) \mathrm{d} x=\left[-\frac{x^{4}}{4}+\frac{5 x^{2}}{2}+2 x\right]_{1}^{2}=\left(-4+10+4-\left(-\frac{1}{4}+\frac{5}{2}+2\right)\right)=\frac{23}{4} .
$$

b)

We see that the function is non-positive on the interval $[-2,-1]$ (green area):

$$
\begin{aligned}
P & =-\int_{-2}^{-1}\left(-x^{3}+5 x+2\right) \mathrm{d} x=\int_{-2}^{-1}\left(x^{3}-5 x-2\right) \mathrm{d} x=\left[\frac{x^{4}}{4}-\frac{5 x^{2}}{2}-2 x\right]_{-2}^{-1} \\
& =\left(\frac{1}{4}-\frac{5}{2}+2-(4-10+4)\right)=\frac{7}{4} .
\end{aligned}
$$

Exercise 45

Sketch the graph of the function $y=\ln \frac{x}{2}$ and find area of the planar region bounded by this graph, $x$-axis and the lines:
a) $x=\frac{1}{2} \quad$ and $\quad x=\frac{3}{2}$
b) $x=2$ and $x=4$ 


\section{Example 46}

Sketch the graph of the function $y=-x^{2}+x+2$ and calculate area of a planar region surrounded by this graph, $x$-axis and the lines $x=-2, x=1$.

We sketch the region whose area we are looking for:

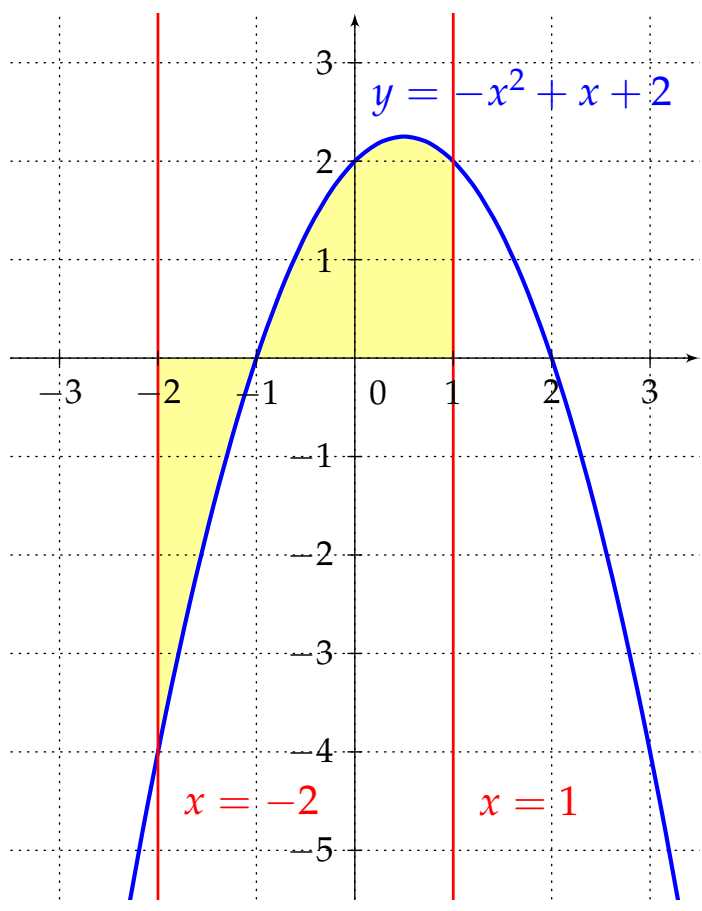

We see that the function changes its sign on $[-2,1]$, therefore we have to find area of the planar region located below $x$-axis and above $x$-axis. At first we find intersection points with $x$-axis on $[-2,1]$ :

$x_{1,2}=\frac{-1 \pm \sqrt{1+8}}{-2} \Rightarrow$ seeking intersection is $x=-1$.

$$
\begin{aligned}
P & =-\int_{-2}^{-1}\left(-x^{2}+x+2\right) \mathrm{d} x+\int_{-1}^{1}\left(-x^{2}+x+2\right) \mathrm{d} x=\left[\frac{x^{3}}{3}-\frac{x^{2}}{2}-2 x\right]_{-2}^{-1}+\left[-\frac{x^{3}}{3}+\frac{x^{2}}{2}+2 x\right]_{-1}^{1} \\
& =\left(-\frac{1}{3}-\frac{1}{2}+2-\left(-\frac{8}{3}-2+4\right)\right)+\left(-\frac{1}{3}+\frac{1}{2}+2-\left(\frac{1}{3}+\frac{1}{2}-2\right)\right)=\frac{11}{6}+\frac{10}{3}=\frac{31}{6}
\end{aligned}
$$

\section{Exercise 47}

Find area of the planar region (draw the region) bounded by $x$-axis and:
a) $y=x^{2}-3, \quad x=-2, \quad x=2$
b) one positive wave of $y=\sin x$

\section{Example 48}

Find area of the planar region surrounded by curves $y=x^{2}-x-2, y=-x+2$. 
We sketch the region whose area we are looking for:

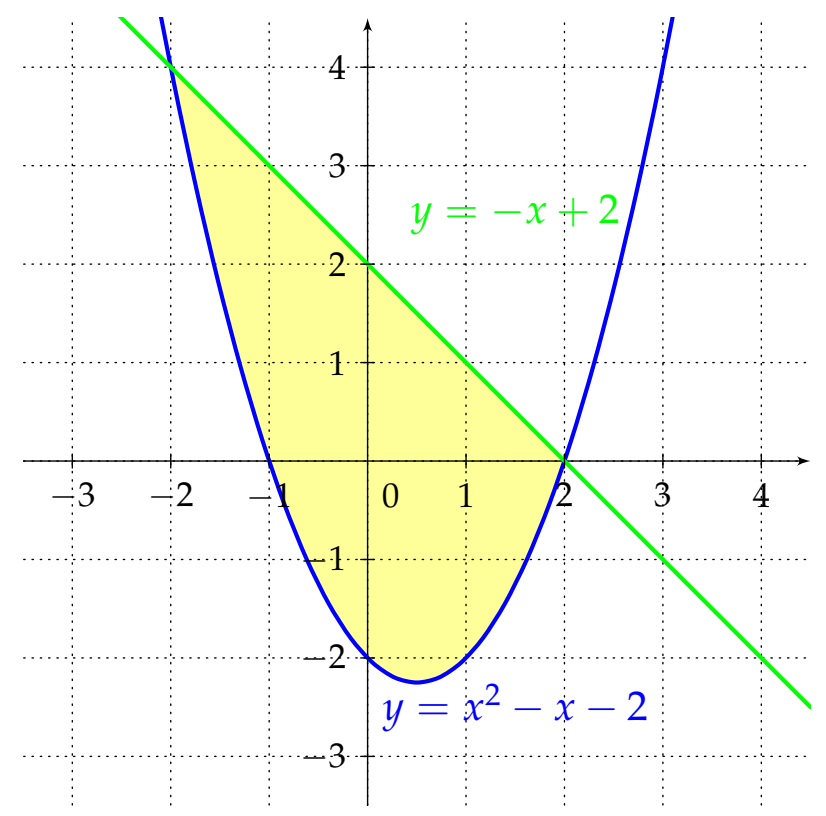

The region is bounded above by the function $y=-x+2$ and bounded below by the quadratic function $y=x^{2}-x-2$, we need to find out integration domain:

$$
\begin{aligned}
& -x+2=x^{2}-x-2 \Rightarrow x^{2}-4=0 \Rightarrow x_{1}=-2, x_{2}=2 \\
P= & -\int_{-2}^{2}\left(-x+2-\left(x^{2}-x-2\right)\right) \mathrm{d} x=\int_{-2}^{2}\left(-x^{2}+4\right) \mathrm{d} x=\left[-\frac{x^{3}}{3}+4 x\right]_{-2}^{2} \\
= & -\frac{8}{3}+8-\left(\frac{8}{3}-8\right)=\frac{32}{3} .
\end{aligned}
$$

Exercise 49

a) Find area of the planar region surrounded by curves $y=\mathrm{e}^{x}, y=\mathrm{e}^{-x}$ and $x=1$.

b) Find area of the planar region surrounded by curves $y=x^{2}+1, y=2 x^{2}-3$.

\section{Example 50}

Find area of the planar regions surrounded by the parametric curve $x=2 a \sin t \cos t$, $y=a \sin t, t \in[0, \pi]$.

We calculate a derivative of $x=\varphi(t)=2 a \sin t \cos t$ :

$$
\dot{\varphi}(t)=2 a\left(\cos ^{2} t-\sin ^{2} t\right)=2 a\left(1-2 \sin ^{2} t\right) .
$$

Result:

$$
\begin{aligned}
P & =\left|\int_{0}^{\pi} 2 a^{2}\left(\sin t-2 \sin ^{3} t\right) \mathrm{d} t\right|=\left|-2 a^{2}[\cos t]_{0}^{\pi}-4 a^{2} \int_{0}^{\pi}\left(1-\cos ^{2} t\right) \sin t \mathrm{~d} t\right| \\
& =\left|4 a^{2}+4 a^{2}\left[\cos t-\frac{\cos ^{3} t}{3}\right]_{0}^{\pi}\right|=\left|4 a^{2}+4 a^{2}\left(-1+\frac{1}{3}-\left(1-\frac{1}{3}\right)\right)\right|=\frac{4}{3} a^{2} .
\end{aligned}
$$


We sketch the region whose area we are looking for:

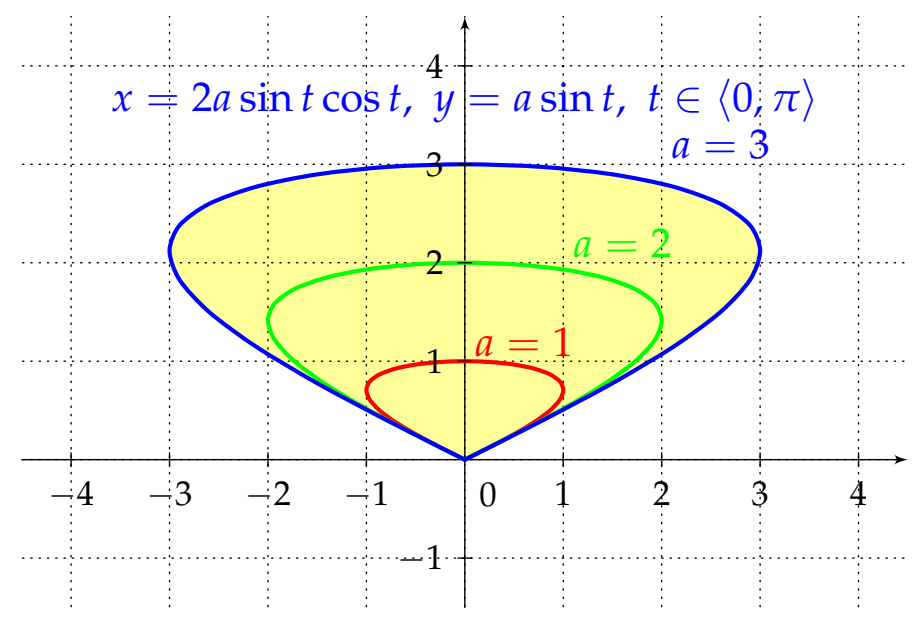

Exercise 51

Find area of the planar region surrounded by the parametric curve $x=2 t-t^{2}, y=$ $2 t^{2}-t^{3}$, where $t \in[0,2]$.

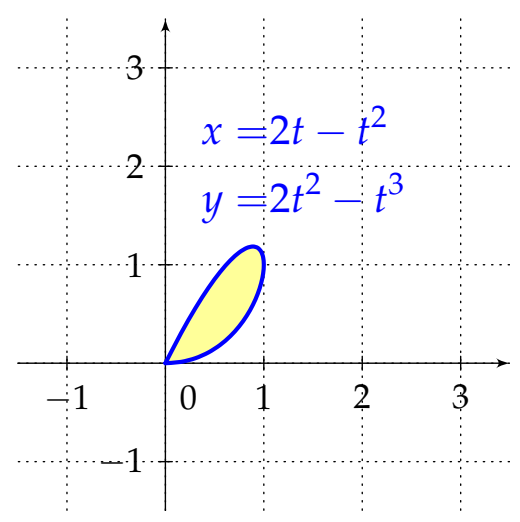

\subsection{Length of planar curves}

Theorem

If a function $y=f(x)$ is defined and has continuous derivative on the interval $[a, b]$, then for length of its graph holds:

$$
l=\int_{a}^{b} \sqrt{1+\left(f^{\prime}(x)\right)^{2}} \mathrm{~d} x
$$

Let us take a closer look on a more general case when a curve does not have to be the graph of a function, e.g. it may be a trajectory of a point continuously moving in the plane. Such curve is represented by parametric equations

$$
x=\varphi(t), y=\psi(t),
$$

where $t \in[\alpha, \beta]$. From the physical point of view length of a curve is a path along which the point passes from time moment $\alpha$ to time moment $\beta$. For length of parametric curves 
holds:

$$
l=\int_{\alpha}^{\beta} \sqrt{(\dot{\varphi}(t))^{2}+(\dot{\psi}(t))^{2}} \mathrm{~d} t
$$

Example 52

Find length of the curve $y=\arcsin x+\sqrt{1-x^{2}}$ for $0 \leq x \leq 1$.

We sketch the curve whose length we are looking for:

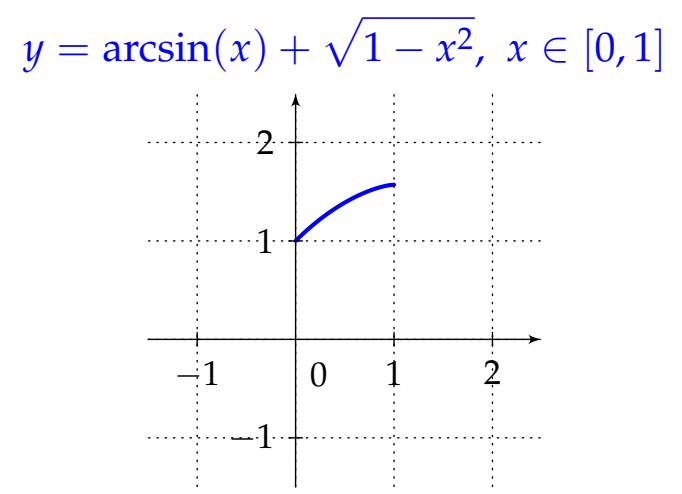

We calculate a derivative and its square:

$$
\left(f^{\prime}(x)\right)^{2}=\left(\frac{1}{\sqrt{1-x^{2}}}-\frac{2 x}{2 \sqrt{1-x^{2}}}\right)^{2}=\frac{1-x}{1+x} .
$$

Result:

$$
\begin{aligned}
l & =\int_{0}^{1} \sqrt{1+\frac{1-x}{1+x}} \mathrm{~d} x=\int_{0}^{1} \sqrt{\frac{1+x+1-x}{1+x}} \mathrm{~d} x=\int_{0}^{1} \frac{\sqrt{2}}{\sqrt{1+x}} \mathrm{~d} x \\
& =\sqrt{2} \int_{0}^{1}(1+x)^{-\frac{1}{2}} \mathrm{~d} x=\sqrt{2}\left[\frac{(1+x)^{\frac{1}{2}}}{\frac{1}{2}}\right]_{0}^{1}=4-2 \sqrt{2} .
\end{aligned}
$$

\section{Exercise 53}

a) Find length of the curve $y^{2}=x^{3}$ on $[0,2]$.

b) Find length of the curve $y=\ln \sin x$ for $\frac{\pi}{4} \leq x \leq \frac{\pi}{2}$.

\section{Example 54}

Find length of trajectory of a point passing from time moment $t=0$ to time moment $t=\sqrt{3}$ along the curve given by parametric equations $x=t^{2}, y=\frac{t}{3}\left(t^{2}-3\right)$. 
We sketch the curve whose length we are looking for:

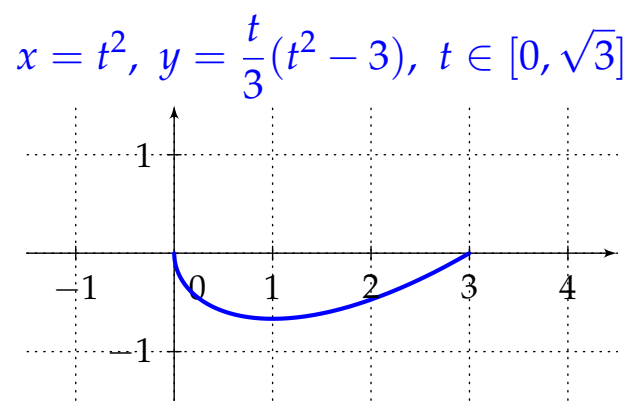

We calculate derivatives:

$$
\begin{aligned}
& \dot{\varphi}(t)=2 t \\
& \dot{\psi}(t)=\frac{t^{2}-3}{3}+\frac{2 t^{2}}{3}=t^{2}-1
\end{aligned}
$$

Result:

$l=\int_{0}^{\sqrt{3}} \sqrt{4 t^{2}+t^{4}-2 t^{2}+1} \mathrm{~d} t=\int_{0}^{\sqrt{3}} \sqrt{t^{4}+2 t^{2}+1} \mathrm{~d} t=\int_{0}^{\sqrt{3}} \sqrt{\left(t^{2}+1\right)^{2}} \mathrm{~d} t=\left[\frac{t^{3}}{3}+t\right]_{0}^{\sqrt{3}}=2 \sqrt{3}$.

\section{Exercise 55}

Find length of a trajectory of the point passing from time moment $t=0$ to time moment $t=2$ along the curve given by parametric equations $x=t^{3}, y=5 t^{2}$.

\subsection{Volume of solids of revolution}

If we rotate a planar region around $x$-axis, then rotation creates a body called solid of revolution whose volume can be found by means of definite integrals.

Theorem

Let a function $y=f(x)$ is continuous and non-negative on the interval $[a, b]$. Then a solid of revolution created by rotation of the planar region surrounded by curve $y=$ $f(x)$ and $x$-axis about $x$-axis has on the interval $[a, b]$ volume:

$$
V=\pi \int_{a}^{b} f^{2}(x) \mathrm{d} x
$$

Remark

1. Analogous formula is valid for rotations about $y$-axis. If the planar region surrounded by the curve $x=h(y)$ and $y$-axis on $[c, d]$ (this interval lies on $y$-axis) rotates about $y$-axis, volume of the solid of revolution is given by:

$$
V=\pi \int_{c}^{d} h^{2}(y) \mathrm{d} y
$$


2. If the solid of revolution is created by means of rotation of a planar region surrounded by curves $y=f(x)$ and $y=g(x)$, whereas it holds $f(x) \geq g(x)$, about $x$-axis on $[a, b]$, then volume of such solid is given by

$$
V=\pi \int_{a}^{b}\left|f^{2}(x)-g^{2}(x)\right| \mathrm{d} x .
$$

Theorem

If a function $f$ is represented by parametric equations $x=\varphi(t), y=\psi(t)$, where $t \in$ $[\alpha, \beta]$, for volume of the solid of revolution created by rotation about $x$-axis holds:

$$
V=\pi \int_{\alpha}^{\beta} \psi^{2}(t)|\dot{\varphi}(t)| \mathrm{d} t .
$$

\section{Example 56}

Find volume of the solid of revolution created by rotation of the planar region surrounded by the curve $y=\ln x$ and $x$-axis on $[1, \mathrm{e}]$ about $x$-axis.

We sketch the planar region we are going to rotate around $x$-axis:
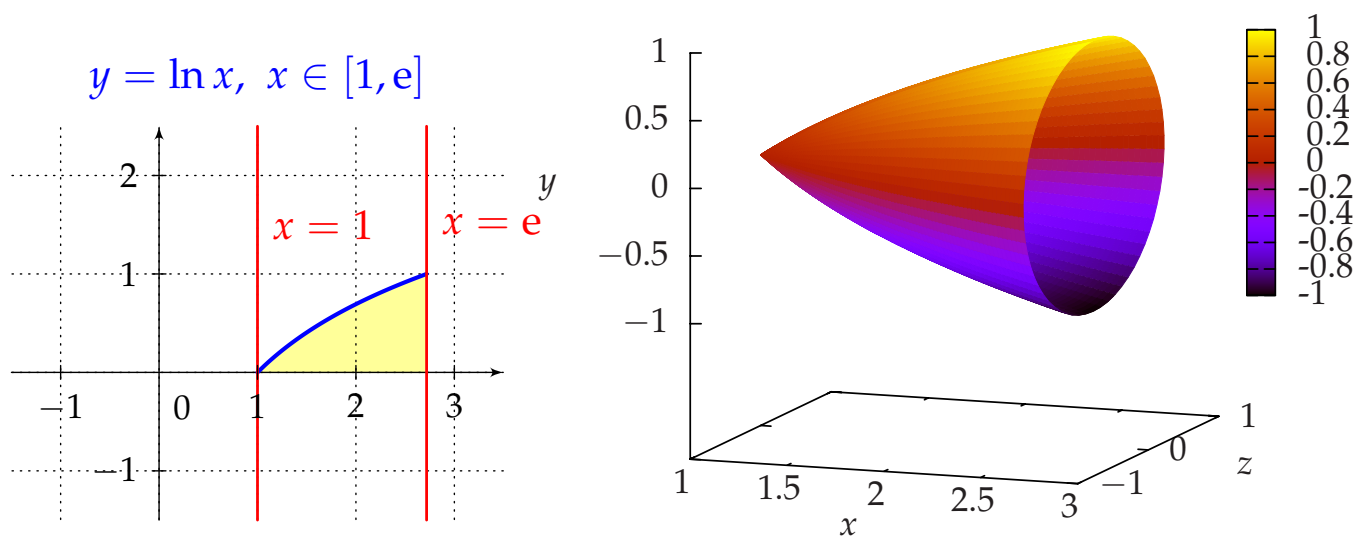

Result:

$$
\begin{aligned}
V & =\pi \int_{1}^{\mathrm{e}} \ln ^{2} x \mathrm{~d} x=\left|\begin{array}{cc}
u=\ln ^{2} x & v^{\prime}=1 \\
u^{\prime}=\frac{2 \ln x}{x} & v=x
\end{array}\right|=\pi\left[x \ln ^{2} x\right]_{1}^{\mathrm{e}}-2 \pi \int_{1}^{\mathrm{e}} \ln x \mathrm{~d} x=\left|\begin{array}{cc}
u=\ln x & v^{\prime}=1 \\
u^{\prime}=\frac{1}{x} & v=x
\end{array}\right| \\
& =\pi \mathrm{e}-2 \pi\left([x \ln x]_{1}^{\mathrm{e}}-\int_{1}^{\mathrm{e}} \mathrm{d} x\right)=\pi \mathrm{e}-2 \pi \mathrm{e}+2 \pi[x]_{1}^{\mathrm{e}}=\pi(\mathrm{e}-2) .
\end{aligned}
$$




\section{Exercise 57}

a) Find volume of the solid of revolution created by rotation of the planar region surrounded by the curve $y=\sqrt{2 x-3}$ and $x$-axis on $[2,3]$ about $x$-axis.

b) Find volume of the solid of revolution created by rotation of the planar region surrounded by the curve $y=2|\sin x|$ and $x$-axis on $[0,2 \pi]$ about $x$-axis.

\section{Example 58}

Find volume of the solid of revolution created by rotation of the planar region surrounded by the curves $y=\mathrm{e}^{x}, y=-2 \mathrm{e}^{-x}+3$ about $x$-axis.

We sketch the planar region we are going to rotate about $x$-axis:
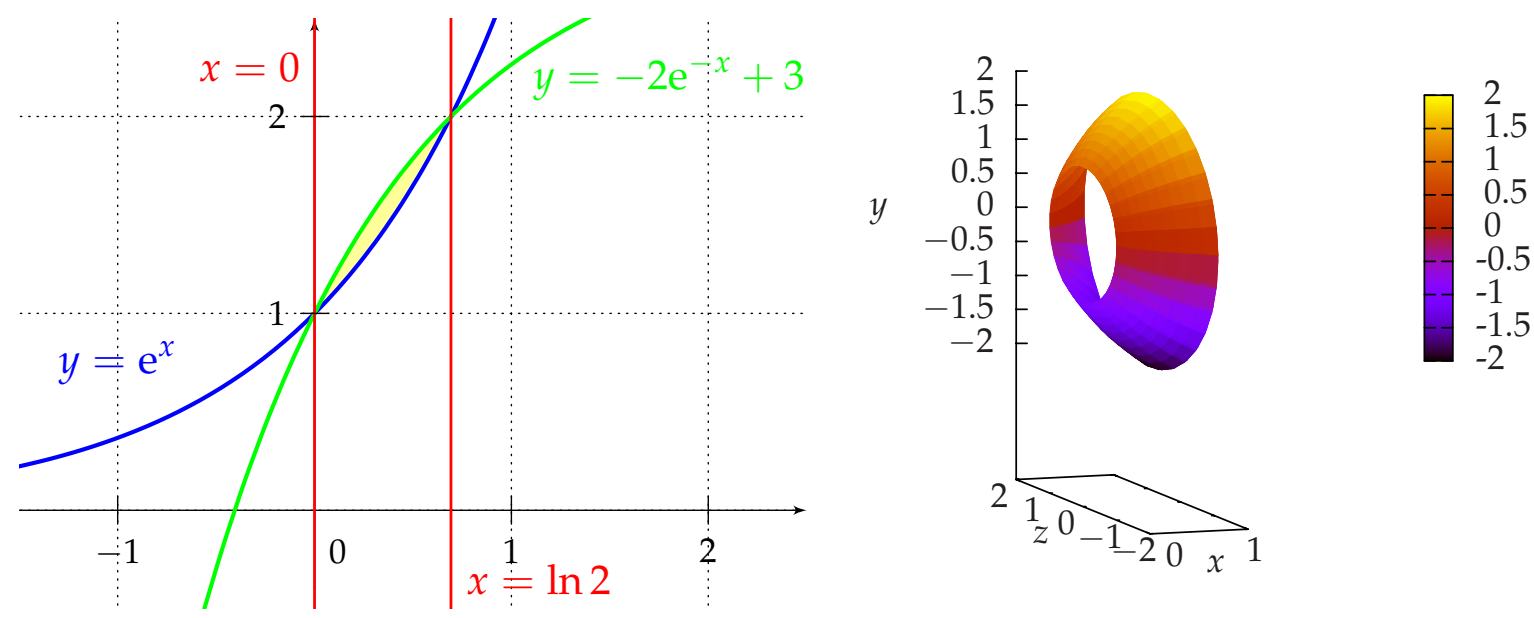

We find intersection points to get integration domain:

$$
-2 \mathrm{e}^{-x}+3=\mathrm{e}^{x} \Rightarrow \frac{2}{\mathrm{e}^{x}}-3+\mathrm{e}^{x}=0 \Rightarrow \frac{2-3 \mathrm{e}^{x}+\mathrm{e}^{2 x}}{\mathrm{e}^{x}}=0 \Rightarrow 2-3 \mathrm{e}^{x}+\mathrm{e}^{2 x}=0,
$$

we use the substitution $\mathrm{e}^{x}=t$ and solve the quadratic equation $t^{2}-3 t+2=0 \Rightarrow x_{1}=$ $0, x_{2}=\ln 2$.

$$
\begin{aligned}
V & =\pi \int_{0}^{\ln 2}\left|\left(-2 \mathrm{e}^{-x}+3\right)^{2}-\mathrm{e}^{2 x}\right| \mathrm{d} x=\pi \int_{0}^{\ln 2}\left(4 \mathrm{e}^{-2 x}-12 \mathrm{e}^{-x}+9-\mathrm{e}^{2 x}\right) \mathrm{d} x \\
& =\pi\left[-2 \mathrm{e}^{-2 x}+12 \mathrm{e}^{-x}+9 x-\frac{\mathrm{e}^{2 x}}{2}\right]_{0}^{\ln 2}=\pi(9 \ln 2-6) .
\end{aligned}
$$




\section{Exercise 59}

a) Find volume of the solid of revolution created by rotation of the planar region surrounded by the curves $y=x^{2}, y^{2}=x$ about $x$-axis.

b) Find volume of the solid of revolution created by rotation of the planar region surrounded by the curves $y=\mathrm{e}^{x}, y=x+2, x=-1, x=1$ about $x$-axis.

\section{Example 60}

Find volume of the solid of revolution created by rotation of the planar region surrounded by the parametric curve $x=\cos ^{2} t, y=\sin ^{2} t$, where $t \in\left[\frac{\pi}{2}, \pi\right]$ about $x$-axis.

We sketch the planar region we are going to rotate around $x$-axis:
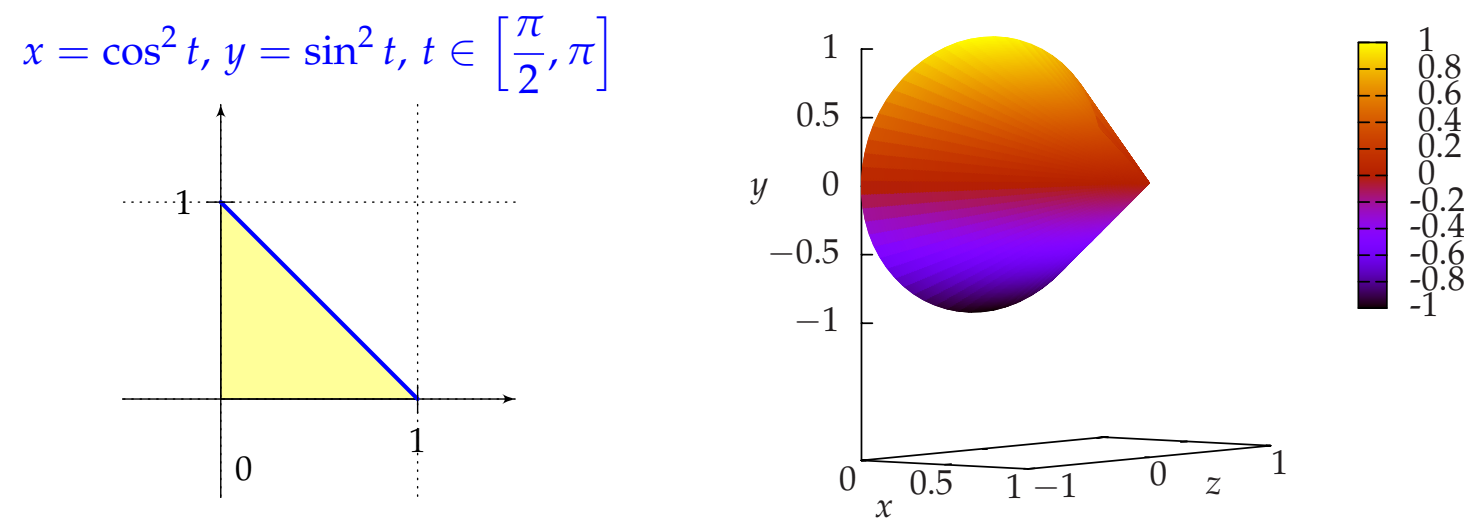

We calculate a derivative of $x=\varphi(t)=\cos ^{2} t$ :

$$
\dot{\varphi}(t)=-2 \cos t \sin t .
$$

Result:

$$
\begin{aligned}
V & =-2 \pi \int_{\frac{\pi}{2}}^{\pi} \cos t \sin ^{5} t \mathrm{~d} t=\left|\begin{array}{c}
\sin t=u \\
\cos t \mathrm{~d} t=\mathrm{d} u \\
t=\frac{\pi}{2} \Rightarrow u=1 \\
t=\pi \Rightarrow u=0
\end{array}\right|=-2 \pi \int_{1}^{0} u^{5} \mathrm{~d} u \\
& =2 \pi \int_{0}^{1} u^{5} \mathrm{~d} u=\frac{1}{3} \pi\left[u^{6}\right]_{0}^{1}=\frac{1}{3} \pi .
\end{aligned}
$$

\section{Exercise 61}

Find volume of the solid of revolution created by rotation of a planar region surrounded by the parametric curve $x=\frac{1-t}{1+t}, y=\frac{1}{1+t}$, where $t \in[0,1]$ about $x$-axis. 


\subsection{Lateral surface of solids of revolution}

\section{Theorem}

Let a function $y=f(x)$ be continuous and non-negative on the interval $[a, b]$ with continuous derivative. Then for lateral surface created by rotation of the curve $y=f(x)$ about $x$-axis and its lateral surface area holds on the interval $[a, b]$ :

$$
S=2 \pi \int_{a}^{b} f(x) \sqrt{1+\left(f^{\prime}(x)\right)^{2}} \mathrm{~d} x .
$$

\section{Remark}

Rotation about $y$-axis:

$$
S=2 \pi \int_{c}^{d} h(y) \sqrt{1+\left(h^{\prime}(y)\right)^{2}} \mathrm{~d} y .
$$

\section{Theorem}

If a function $y=f(x)$ is represented by parametric equations $x=\varphi(t), y=\psi(t)$, where $t \in[\alpha, \beta]$, for lateral surface created by rotation of such function about $x$-axis and its lateral surface area holds:

$$
S=2 \pi \int_{\alpha}^{\beta} \psi(t) \sqrt{(\dot{\varphi}(t))^{2}+(\dot{\psi}(t))^{2}} \mathrm{~d} t, \psi(t) \geq 0 .
$$

\section{Example 62}

Find lateral surface area of the solid revolution created by rotation of the planar region surrounded by the curve $y=2 \sqrt{x}$ and $x$-axis on $x \in[0,2]$ about $x$-axis.

We sketch the planar region we are going to rotate about $x$-axis:
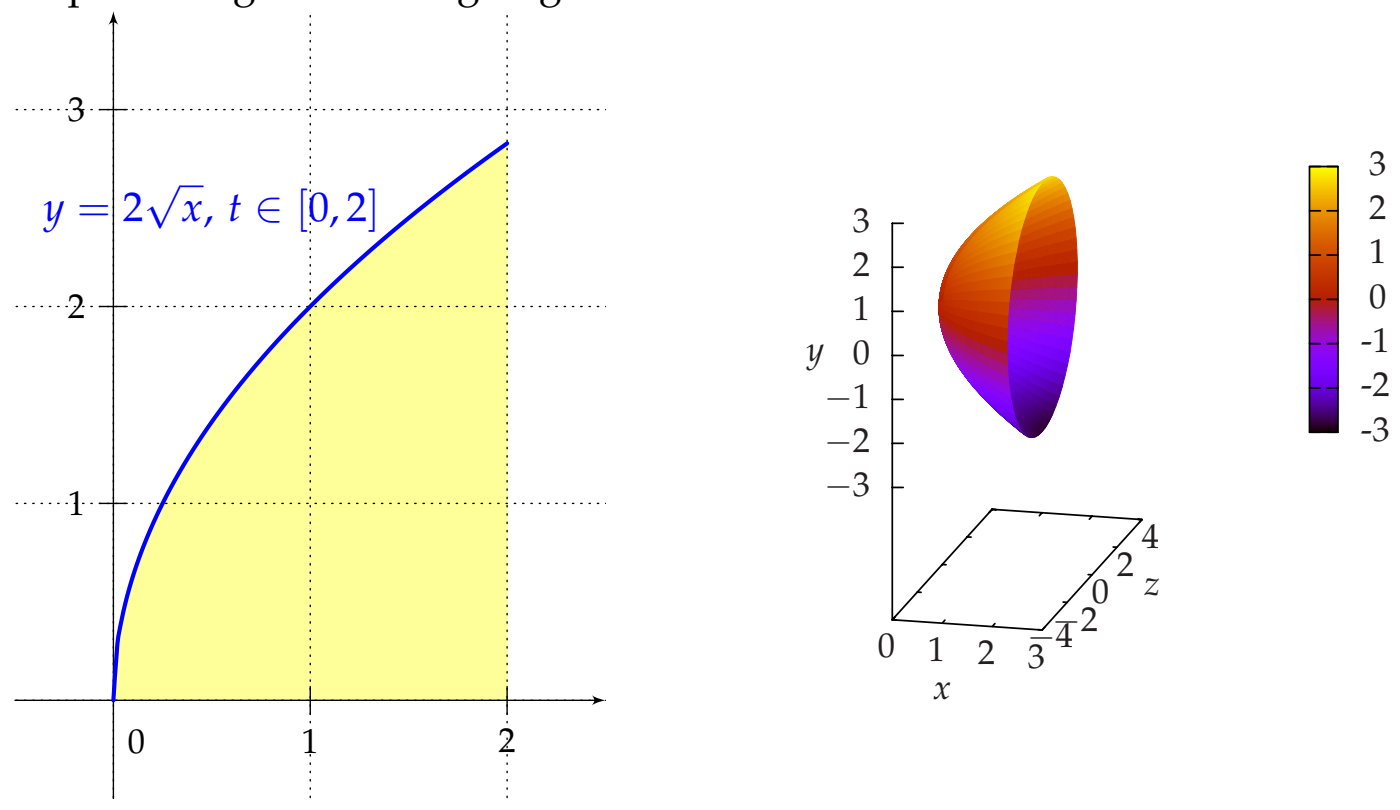
We calculate the second power of a derivative:

$$
\left(y^{\prime}\right)^{2}=\frac{1}{x}
$$

Result:

$$
\begin{aligned}
S & =4 \pi \int_{0}^{2} \sqrt{x} \sqrt{1+\frac{1}{x}} \mathrm{~d} x=4 \pi \int_{0}^{2} \sqrt{x} \sqrt{\frac{x+1}{x}} \mathrm{~d} x=4 \pi \int_{0}^{2} \sqrt{x+1} \mathrm{~d} x \\
& =\frac{8}{3} \pi\left[(x+1)^{\frac{3}{2}}\right]_{0}^{2}=\frac{8}{3} \pi(\sqrt{27}-1) .
\end{aligned}
$$

\section{Exercise 63}

Find lateral surface area of the solid of revolution created by rotation of the planar region surrounded by the curve $y=\sqrt{x}$ and $x$-axis on $[1,4]$ about $x$-axis.

\section{Example 64}

Find lateral surface area of the solid of revolution created by rotation of the planar region surrounded by the parametric curve $x=\cos ^{2} t, y=\sin ^{2} t$ on $\left[0, \frac{\pi}{2}\right]$ about $x$-axis.

We sketch the planar region we are going to rotate about $x$-axis:

$$
x=\cos ^{2} t, y=\sin ^{2} t, t \in\left[\frac{\pi}{2}, \pi\right]
$$
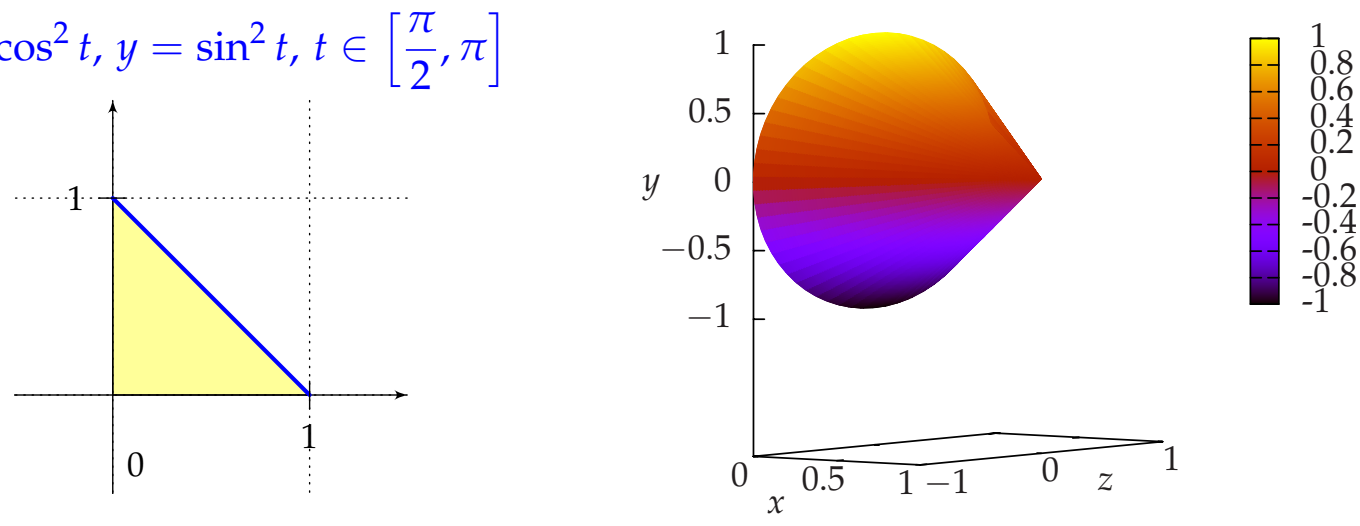

We calculate second powers of derivatives:

$$
\begin{aligned}
& (\dot{\varphi}(t))^{2}=4 \cos ^{2} t \sin ^{2} t \\
& (\dot{\psi}(t))^{2}=4 \cos ^{2} t \sin ^{2} t
\end{aligned}
$$

Result:

$$
\begin{aligned}
S & =2 \pi \int_{0}^{\frac{\pi}{2}} \sin ^{2} t \sqrt{8 \cos ^{2} t \sin ^{2} t} \mathrm{~d} t=2 \pi \sqrt{8} \int_{0}^{\frac{\pi}{2}} \cos t \sin ^{3} t \mathrm{~d} t \\
& =\left|\begin{array}{c}
\sin t=u \\
\cos t \mathrm{~d} t=\mathrm{d} u \\
t=0 \Rightarrow u=0 \\
t=\frac{\pi}{2} \Rightarrow u=1
\end{array}\right|=2 \pi \sqrt{8} \int_{0}^{1} u^{3} \mathrm{~d} u=\pi \sqrt{2}\left[u^{4}\right]_{0}^{1}=\pi \sqrt{2} .
\end{aligned}
$$




\section{Exercise 65}

Find lateral surface area of the solid of revolution created by rotation of the planar region surrounded by the parametric curve $x=a \sin 2 t, y=2 a \sin ^{2} t$ on $[0, \pi]$ about $x$-axis.

\subsection{Physical applications, mass center and moments}

Let us consider a point $A=[x, y]$ with mass $m$ and arbitrary $a$-axis (line) in coordinate plane. Denote $r$ distance of the point $A$ from $a$-axis. Moment about $a$-axis is given by

$$
S_{a}=m r
$$

Moment of inertia about $a$-axis is given by

$$
I_{a}=m r^{2}
$$

If we deal with coordinate axes $x, y$ as axes of rotation then we get the following formulas for moments

$$
S_{x}=m y, \quad S_{y}=m x, \quad I_{x}=m y^{2}, \quad I_{y}=m x^{2} .
$$

Let us consider a system of $n$ points $A_{i}$ with masses $m_{i}, i=1,2, \ldots, n$. Then we have the following objects

total mass of the system

$$
m=\sum_{i=1}^{n} m_{i} \quad n \rightarrow \infty \quad m=\lim _{n \rightarrow \infty} \sum_{i=1}^{n} m_{i}=\int_{a}^{b} \mathrm{~d} m
$$

moment about the $x$-axis

$$
S_{x}=\sum_{i=1}^{n} m_{i} y_{i} \quad n \rightarrow \infty \quad S_{x}=\lim _{n \rightarrow \infty} \sum_{i=1}^{n} m_{i} y_{i}=\int_{a}^{b} y \mathrm{~d} m
$$

moment about the $y$-axis

$$
S_{y}=\sum_{i=1}^{n} m_{i} x_{i} \quad n \rightarrow \infty \quad S_{y}=\lim _{n \rightarrow \infty} \sum_{i=1}^{n} m_{i} x_{i}=\int_{a}^{b} x \mathrm{~d} m
$$

moment of inertia about the $x$-axis $\quad I_{x}=\sum_{i=1}^{n} m_{i} y_{i}^{2} \quad n \rightarrow \infty \quad I_{x}=\lim _{n \rightarrow \infty} \sum_{i=1}^{n} m_{i} y_{i}^{2}=\int_{a}^{b} y^{2} \mathrm{~d} m$ moment of inertia about the $y$-axis $\quad I_{y}=\sum_{i=1}^{n} m_{i} x_{i}^{2} \quad n \rightarrow \infty \quad I_{y}=\lim _{n \rightarrow \infty} \sum_{i=1}^{n} m_{i} x_{i}^{2}=\int_{a}^{b} x^{2} \mathrm{~d} m$ $[a, b]$ is a given interval, $\mathrm{d} m$ is elementary mass.

Center of mass $T=\left[x_{T}, y_{T}\right]$ of the system of mass points is the point with the following property: if one focuses entire mass of the system of mass points into this point $T$ then its moments about coordinate axes are the same as moments of the original system of mass points. Thus

$$
m x_{T}=S_{y}, m y_{T}=S_{x} \Rightarrow x_{T}=\frac{S_{y}}{m}, y_{T}=\frac{S_{x}}{m} .
$$

From physics we know how to calculate mass $m$ of an object, simply multiply density and volume, $m=\rho \cdot V$. For a planar region we have $m=\rho \cdot P$, where $P$ is its area. For a planar curve it holds $m=\rho \cdot l$, where $l$ is its length. Be very careful to use correct units. 


\subsection{Homogeneous curves}

Theorem

Let a curve is given by explicit equation $y=f(x)$ with continuous derivative $f^{\prime}(x)$ on $[a, b]$. Let density $\rho$ of the curve is constant. Mass of the curve is given by

$$
m=\int_{a}^{b} \mathrm{~d} m=\rho \int_{a}^{b} \mathrm{~d} l=\rho \int_{a}^{b} \sqrt{1+\left(f^{\prime}(x)\right)^{2}} \mathrm{~d} x .
$$

For moments about coordinate axes hold,

$$
\begin{aligned}
& S_{x}=\int_{a}^{b} y \mathrm{~d} m=\rho \int_{a}^{b} f(x) \sqrt{1+\left(f^{\prime}(x)\right)^{2}} \mathrm{~d} x, \\
& S_{y}=\int_{a}^{b} x \mathrm{~d} m=\rho \int_{a}^{b} x \sqrt{1+\left(f^{\prime}(x)\right)^{2}} \mathrm{~d} x .
\end{aligned}
$$

For moments of inertia about coordinate axes hold,

$$
\begin{aligned}
& I_{x}=\int_{a}^{b} y^{2} \mathrm{~d} m=\rho \int_{a}^{b} f^{2}(x) \sqrt{1+\left(f^{\prime}(x)\right)^{2}} \mathrm{~d} x, \\
& I_{y}=\int_{a}^{b} x^{2} \mathrm{~d} m=\rho \int_{a}^{b} x^{2} \sqrt{1+\left(f^{\prime}(x)\right)^{2}} \mathrm{~d} x .
\end{aligned}
$$

Mass center coordinates, $T=\left[x_{T}, y_{T}\right]$

$$
x_{T}=\frac{S_{y}}{m}=\frac{\int_{a}^{b} x \sqrt{1+\left(f^{\prime}(x)\right)^{2}} \mathrm{~d} x}{\int_{a}^{b} \sqrt{1+\left(f^{\prime}(x)\right)^{2}} \mathrm{~d} x}, y_{T}=\frac{S_{x}}{m}=\frac{\int_{a}^{b} f(x) \sqrt{1+\left(f^{\prime}(x)\right)^{2}} \mathrm{~d} x}{\int_{a}^{b} \sqrt{1+\left(f^{\prime}(x)\right)^{2}} \mathrm{~d} x} .
$$




\section{Theorem}

Consider a curve represented by parametric equations $x=\varphi(t), y=\psi(t)$ on $[\alpha, \beta]$, $\psi(t) \geq 0$. The functions $\varphi(t), \psi(t)$ have continuous derivatives on $[\alpha, \beta], \dot{\varphi}(t)>0$. If density $\rho$ is constant, then the curve has mass

$$
m=\int_{\alpha}^{\beta} \mathrm{d} m=\rho \int_{\alpha}^{\beta} \mathrm{d} l=\rho \int_{\alpha}^{\beta} \sqrt{\dot{\varphi}^{2}(t)+\dot{\psi}^{2}(t)} \mathrm{d} t .
$$

For moments about coordinate axes hold,

$$
\begin{aligned}
& S_{x}=\int_{\alpha}^{\beta} y \mathrm{~d} m=\rho \int_{\alpha}^{\beta} \psi(t) \sqrt{\dot{\varphi}^{2}(t)+\dot{\psi}^{2}(t)} \mathrm{d} t, \\
& S_{y}=\int_{\alpha}^{\beta} x \mathrm{~d} m=\rho \int_{\alpha}^{\beta} \varphi(t) \sqrt{\dot{\varphi}^{2}(t)+\dot{\psi}^{2}(t)} \mathrm{d} t .
\end{aligned}
$$

For moments of inertia about coordinate axes hold,

$$
\begin{aligned}
& I_{x}=\int_{\alpha}^{\beta} y^{2} \mathrm{~d} m=\rho \int_{\alpha}^{\beta} \psi^{2}(t) \sqrt{\dot{\varphi}^{2}(t)+\dot{\psi}^{2}(t)} \mathrm{d} t, \\
& I_{y}=\int_{\alpha}^{\beta} x^{2} \mathrm{~d} m=\rho \int_{\alpha}^{\beta} \varphi^{2}(t) \sqrt{\dot{\varphi}^{2}(t)+\dot{\psi}^{2}(t)} \mathrm{d} t .
\end{aligned}
$$

Mass center coordinates, $T=\left[x_{T}, y_{T}\right], x_{T}=S_{y} / m, y_{T}=S_{x} / m$,

$$
x_{T}=\frac{\int_{\alpha}^{\beta} \varphi(t) \sqrt{\dot{\varphi}^{2}(t)+\dot{\psi}^{2}(t)} \mathrm{d} t}{\int_{\alpha}^{\beta} \sqrt{\dot{\varphi}^{2}(t)+\dot{\psi}^{2}(t)} \mathrm{d} t}, y_{T}=\frac{\int_{\alpha}^{\beta} \psi(t) \sqrt{\dot{\varphi}^{2}(t)+\dot{\psi}^{2}(t)} \mathrm{d} t}{\int_{\alpha}^{\beta} \sqrt{\dot{\varphi}^{2}(t)+\dot{\psi}^{2}(t)} \mathrm{d} t} .
$$

\section{Example 66}

Find center of mass of homogeneous half-circle represented by parametric equations $x=r \cos t, y=r \sin t, t \in[0, \pi]$.

We sketch the curve whose center of mass we are looking for:

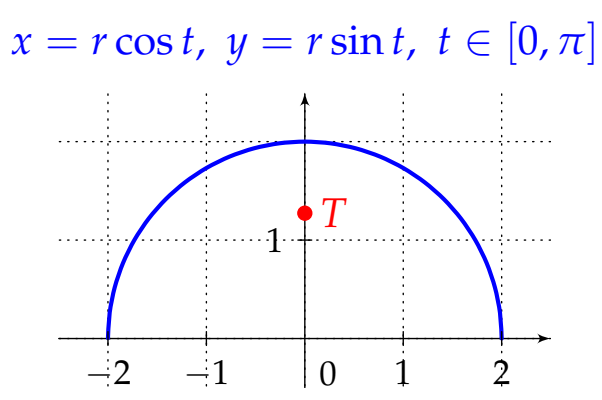


We calculate derivatives:

$$
\dot{\varphi}(t)=-r \sin t, \quad \dot{\psi}(t)=r \cos t
$$

We calculate mass of the curve:

$$
m=\rho \int_{0}^{\pi} \sqrt{r^{2} \sin ^{2} t+r^{2} \cos ^{2} t} \mathrm{~d} t=\rho \int_{0}^{\pi} \sqrt{r^{2}\left(\sin ^{2} t+\cos ^{2} t\right)} \mathrm{d} t=\rho r \int_{0}^{\pi} \mathrm{d} t=\rho r[t]_{0}^{\pi}=\rho \pi r .
$$

For moments about coordinate axes we have:

$$
\begin{aligned}
& S_{x}=\rho \int_{0}^{\pi} r \sin t \sqrt{r^{2} \sin ^{2} t+r^{2} \cos ^{2} t} \mathrm{~d} t=\rho r^{2} \int_{0}^{\pi} \sin t \mathrm{~d} t=\rho r^{2}[-\cos t]_{0}^{\pi}=2 \rho r^{2}, \\
& S_{y}=\rho \int_{0}^{\pi} r \cos t \sqrt{r^{2} \sin ^{2} t+r^{2} \cos ^{2} t} \mathrm{~d} t=\rho r^{2} \int_{0}^{\pi} \cos t \mathrm{~d} t=\rho r^{2}[\sin t]_{0}^{\pi}=0 .
\end{aligned}
$$

Center of mass coordinates:

$$
x_{T}=\frac{S_{y}}{m}=\frac{0}{\rho \pi r}=0, \quad y_{T}=\frac{S_{x}}{m}=\frac{2 \rho r^{2}}{\rho \pi r}=\frac{2 r}{\pi} \Rightarrow T=\left[0, \frac{2 r}{\pi}\right] .
$$

Exercise 67

a) Find center of mass of the homogeneous curve represented by parametric equations $x=t^{2}, y=t-\frac{t^{3}}{3}, t \in[0, \sqrt{3}]$.

b) Find center of mass of the homogeneous curve given by $f(x)=-\frac{x^{2}}{2}+2$ on $[-2,2]$. 


\subsection{Homogeneous regions}

Theorem

Let a region is surrounded by the curves $y=f(x), y=g(x), x=a, x=b$ and $f(x) \geq$ $g(x) \forall x \in[a, b]$. Let density $\rho$ of the region is constant. Mass of the region is given by

$$
m=\int_{a}^{b} \mathrm{~d} m=\rho \int_{a}^{b} \mathrm{~d} P=\rho \int_{a}^{b}(f(x)-g(x)) \mathrm{d} x .
$$

For moments about coordinate axes hold,

$$
\begin{aligned}
& S_{x}=\int_{a}^{b} y \mathrm{~d} m=\frac{\rho}{2} \int_{a}^{b}\left(f^{2}(x)-g^{2}(x)\right) \mathrm{d} x, \\
& S_{y}=\int_{a}^{b} x \mathrm{~d} m=\rho \int_{a}^{b} x(f(x)-g(x)) \mathrm{d} x .
\end{aligned}
$$

For moments of inertia about coordinate axes hold,

$$
\begin{aligned}
& I_{x}=\int_{a}^{b} y^{2} \mathrm{~d} m=\frac{\rho}{3} \int_{a}^{b}\left(f^{3}(x)-g^{3}(x)\right) \mathrm{d} x, \\
& I_{y}=\int_{a}^{b} x^{2} \mathrm{~d} m=\rho \int_{a}^{b} x^{2}(f(x)-g(x)) \mathrm{d} x .
\end{aligned}
$$

Mass center coordinates, $T=\left[x_{T}, y_{T}\right]$

$$
x_{T}=\frac{S_{y}}{m}=\frac{\int_{a}^{b} x(f(x)-g(x)) \mathrm{d} x}{\int_{a}^{b}(f(x)-g(x)) \mathrm{d} x}, y_{T}=\frac{S_{x}}{m}=\frac{\frac{1}{2} \int_{a}^{b}\left(f^{2}(x)-g^{2}(x)\right) \mathrm{d} x}{\int_{a}^{b}(f(x)-g(x)) \mathrm{d} x} .
$$




\section{Theorem}

Consider a region represented by parametric equations $x=\varphi(t), y=\psi(t)$ on $[\alpha, \beta]$, the function $\varphi(t)$ has continuous positive derivative on $[\alpha, \beta], \psi(t) \geq 0$. If density $\rho$ is constant, then the region has mass

$$
m=\int_{\alpha}^{\beta} \mathrm{d} m=\rho \int_{\alpha}^{\beta} \mathrm{d} P=\rho \int_{\alpha}^{\beta} \psi(t) \dot{\varphi}(t) \mathrm{d} t .
$$

For moments about coordinate axes hold,

$$
\begin{aligned}
& S_{x}=\int_{\alpha}^{\beta} y \mathrm{~d} m=\frac{\rho}{2} \int_{\alpha}^{\beta} \psi^{2}(t) \dot{\varphi}(t) \mathrm{d} t, \\
& S_{y}=\int_{\alpha}^{\beta} x \mathrm{~d} m=\rho \int_{\alpha}^{\beta} \varphi(t) \psi(t) \dot{\varphi}(t) \mathrm{d} t .
\end{aligned}
$$

For moments of inertia about coordinate axes hold,

$$
\begin{aligned}
& I_{x}=\int_{\alpha}^{\beta} y^{2} \mathrm{~d} m=\frac{\rho}{3} \int_{\alpha}^{\beta} \psi^{3}(t) \dot{\varphi}(t) \mathrm{d} t, \\
& I_{y}=\int_{\alpha}^{\beta} x^{2} \mathrm{~d} m=\rho \int_{\alpha}^{\beta} \varphi^{2}(t) \psi(t) \dot{\varphi}(t) \mathrm{d} t .
\end{aligned}
$$

Mass center coordinates, $T=\left[x_{T}, y_{Y}\right]$

$$
x_{T}=\frac{S_{y}}{m}=\frac{\int_{\alpha}^{\beta} \varphi(t) \psi(t) \dot{\varphi}(t) \mathrm{d} t}{\int_{\alpha}^{\beta} \psi(t) \dot{\varphi}(t) \mathrm{d} t}, y_{T}=\frac{S_{x}}{m}=\frac{\frac{1}{2} \int_{\alpha}^{\beta} \psi^{2}(t) \dot{\varphi}(t) \mathrm{d} t}{\int_{\alpha}^{\beta} \psi(t) \dot{\varphi}(t) \mathrm{d} t} .
$$




\section{Example 68}

Find center of mass of the homogeneous region surrounded by curves $y^{2}=4 x, x=2$.

$$
\begin{aligned}
y^{2} & =4 x \\
|y| & =2 \sqrt{x} \\
y & = \pm 2 \sqrt{x}
\end{aligned}
$$

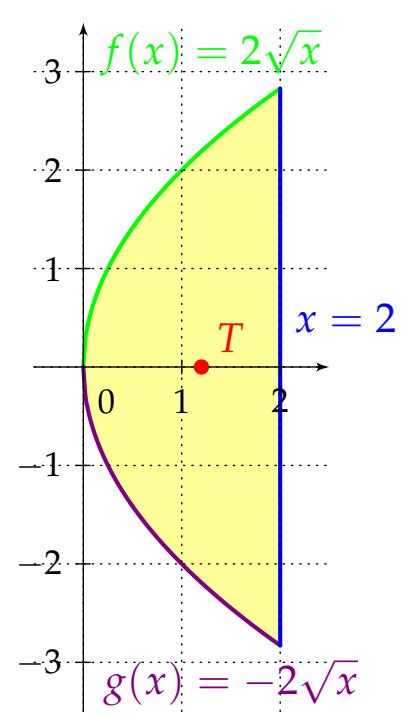

We calculate mass of the region:

$$
m=\rho \int_{0}^{2}(2 \sqrt{x}-(-2 \sqrt{x})) \mathrm{d} x=\rho \int_{0}^{2} 4 \sqrt{x} \mathrm{~d} x=\rho \frac{8}{3}\left[\sqrt{x^{3}}\right]_{0}^{2}=\rho \frac{16}{3} \sqrt{2}
$$

For moments about coordinate axes we have:

$$
\begin{aligned}
& S_{x}=\frac{\rho}{2} \int_{0}^{2}(4 x-4 x) \mathrm{d} x=0, \\
& S_{y}=\rho \int_{0}^{2} x(2 \sqrt{x}-(-2 \sqrt{x})) \mathrm{d} x=4 \rho \int_{0}^{2} x \sqrt{x} \mathrm{~d} x=\rho \frac{8}{5}\left[\sqrt{x^{5}}\right]_{0}^{2}=\rho \frac{32}{5} \sqrt{2} .
\end{aligned}
$$

Center of mass coordinates:

$$
x_{T}=\frac{S_{y}}{m}=\frac{\rho \frac{32}{5} \sqrt{2}}{\rho \frac{16}{3} \sqrt{2}}=\frac{6}{5}, \quad y_{T}=\frac{S_{x}}{m}=0 \Rightarrow T=\left[\frac{6}{5}, 0\right] .
$$

\section{Exercise 69}

a) Find center of mass of the homogeneous region surrounded by curves $y=\sin x$, $y=0,0 \leq x \leq \pi$

b) Find center of mass of the homogeneous region surrounded by the curve represented by parametric equations $x=4 t^{2}-4 t, y=-5 t^{3}+5 t^{2}, 0 \leq t \leq 1$. 


\section{Differential calculus of functions of two real variables}

\subsection{Functions of two real variables, domains}

Definition

Let $M \neq \varnothing, M \subseteq \mathbb{R}^{2}$. By function of two real variables we mean a mapping

$$
f: M \rightarrow \mathbb{R}, \quad M \ni[x, y] \mapsto z=f(x, y) \in \mathbb{R} .
$$

The set $M$ is called domain of the function $f$ and it is denoted by $D_{f}$.

The set $\mathbb{R}^{2}$ is a result of the Cartesian product of the set $\mathbb{R}$ with itself, i.e. $\mathbb{R}^{2}=\mathbb{R} \times \mathbb{R}$. Elements of $\mathbb{R}^{2}$ and also elements of the subset $M$ are called ordered pairs.

Remark

- In analogy with the notation $y=f(x)$ used for functions of one real variable, we use $z=f(x, y)$ for functions of two real variables.

- The variables $x$ and $y$ are independent variables. The variable $z$ is dependent variable.

- If the domain is not specified, we automatically mean that a function is defined on the maximal admissible subset of $\mathbb{R}^{2}$.

- The definition can be easily generalized for functions depending on three or more variables, simply by adding additional independent variables.

- An element of the domain $M$ is called point. Usually we denote points belonging to a domain by capital letters, $A=\left[x_{0}, y_{0}\right]$.

- The value $z=f(A)=f\left(x_{0}, y_{0}\right)$ is called function value.

Example 70

Find and sketch the domain of the function $z=\frac{\ln (1-x)}{\sqrt{16-x^{2}-y^{2}}}$.

The condition $1-x>0$ ensures existence of logarithm $\Rightarrow x<1$. The geometrical interpretation of the preceding inequality is as follows. It is a halfplane with the border line $x=1$, but points on this line do not belong to the domain, i.e. we use dashed style for this border line.

The condition $16-x^{2}-y^{2} \geq 0$ ensures existence of the square root function, the condition $\sqrt{16-x^{2}-y^{2}} \neq 0$ guarantees not dividing by zero. These two conditions can be joined into the one, $16-x^{2}-y^{2}>0 \Rightarrow x^{2}+y^{2}<4^{2}$. This inequality represents a circle with the center at the origin and radius equals to 4 . Points belonging to the border circle must be excluded from domain, i.e. dashed style must be used. 
Finally, the domain is the intersection of both regions, yellow marked region on the picture.

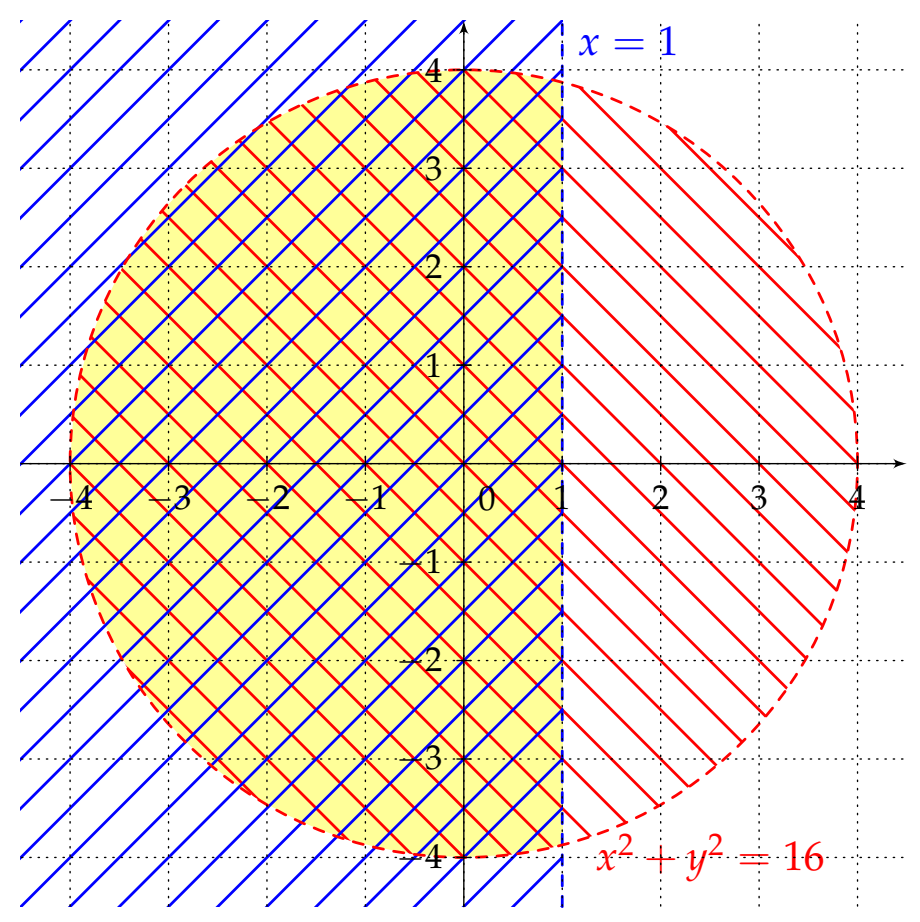

\section{Example 71}

Find and sketch the domain of the function $z=\sqrt{y \sin x}$.

The condition $y \sin x \geq 0$ guarantees existence of the square root function. The product $y \sin x$ is non-negative if and only if both factors are non-negative or non-positive, thus

$$
(y \geq 0 \wedge \sin x \geq 0) \vee(y \leq 0 \wedge \sin x \leq 0) .
$$

Finally, we need to discus a solution of the inequalities $\sin x \geq 0$ and $\sin x \leq 0$. We are looking for the points in which the sine function is either non-negative or non-positive.

$$
\sin x \geq 0 \Rightarrow x \in \bigcup_{k \in \mathbb{Z}}[0+k 2 \pi, \pi+k 2 \pi]
$$

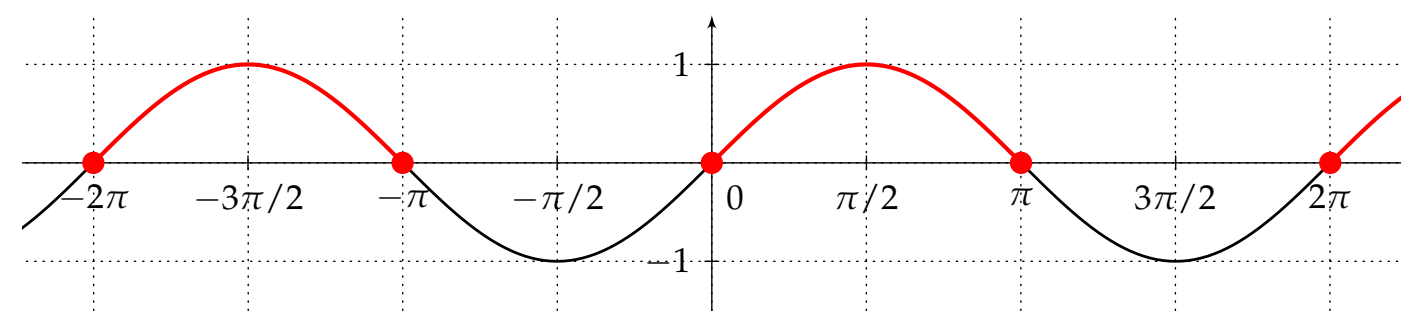

$$
\sin x \leq 0 \Rightarrow x \in \bigcup_{k \in \mathbb{Z}}[-\pi+k 2 \pi, 0+k 2 \pi]
$$

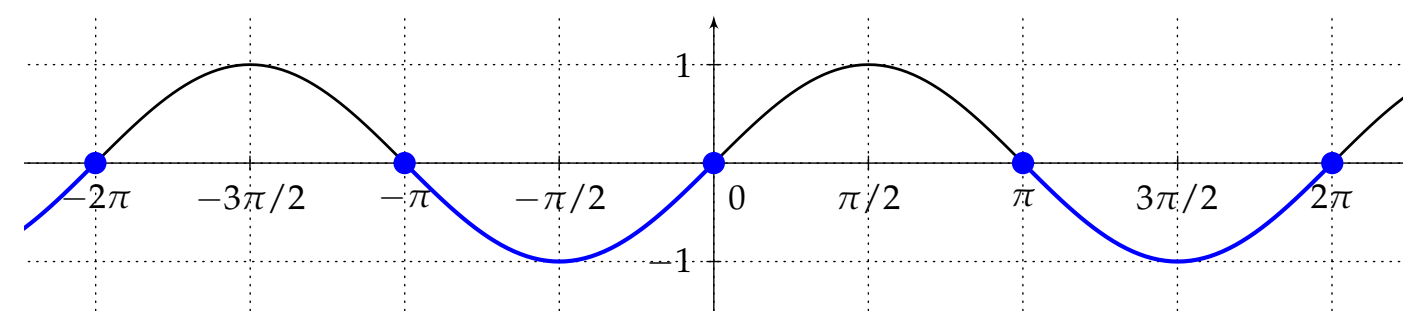




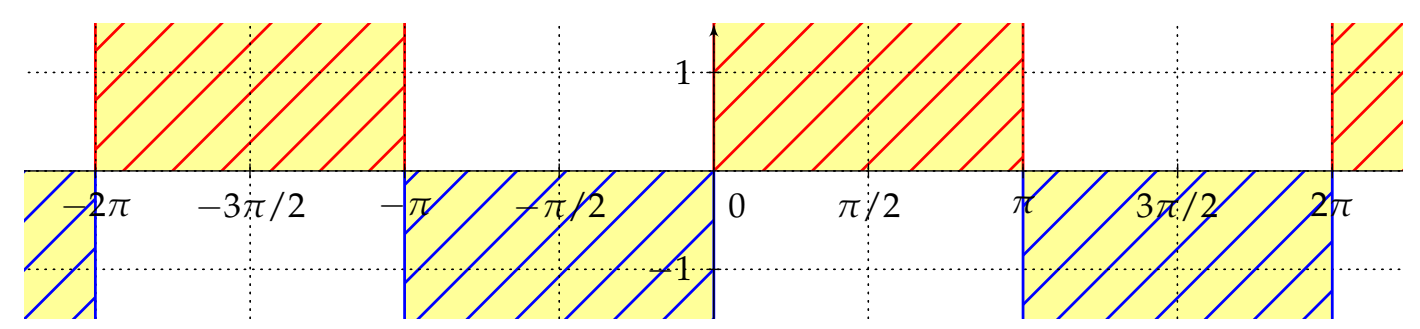

\section{Exercise 72}

Find the domain of the function:
a) $z=\sqrt{2 x+y}$
f) $z=\ln (x y-4)$
b) $z=\frac{x+2 y}{\sqrt{y^{2}-4}}+\sqrt{x^{2}-1}$
g) $z=\arccos \left(2 x^{2}+\frac{2 y^{2}}{9}-1\right)$
c) $z=\ln (y(2-x))$
h) $z=\sqrt{\cos (x-y)}$
d) $z=\sqrt{16-x^{2}-y^{2}}$
i) $z=\arcsin \frac{x^{2}+y^{2}-5}{4}$
e) $z=\frac{1}{\arcsin x \arccos 2 y}$
j) $z=\sqrt{1-x^{2}+y}+\sqrt{1-x^{2}-y}$

\subsection{Functions of two real variables, graphs}

\section{Definition}

Graph of a function of two real variables is the set

$$
G_{f}=\left\{[x, y, z] \in \mathbb{R}^{3} \mid[x, y] \in D_{f}, z=f(x, y)\right\} .
$$

Remark

- The set $G_{f}$ is a subset in $\mathbb{R}^{3}, G_{f} \subset \mathbb{R}^{3}$. Most often we work with such functions that their graphs are represented by 2-dimensional surfaces in 3-dimensional space.

- To draw the graph of a function "manually" is difficult in general and mostly it cannot be done. One crucial option for the visualization of graphs is to use intersection curves of the given graph with coordinate planes and their parallel planes, especially with $x y$-plane.

- For the visualization of graphs one can use computers and appropriate software (GeoGebra, Gnuplot, Maple, Matlab, WolframAlpha etc.).

- The graph of a function depending on three variables is represented by the surface in 4-dimensional space $\mathbb{R}^{4}$, called hypersurface. Such graph cannot be in our 3dimensional space visualized. 


\subsection{Contour lines}

\section{Definition}

Sections of the graph of a function $z=f(x, y)$ and planes parallel with the coordinate $x y$-plane are called contours or contour lines. By contour graph we shall mean the projection of the contour lines into the $x y$-plane $z=0$.

A contour line is a set of points belonging to the graph of a function with the same value. One can meat contours especially on touristic maps (usually gray curves), where they represent the points with the same altitude. On the picture one can see surroundings of VSBTUO.

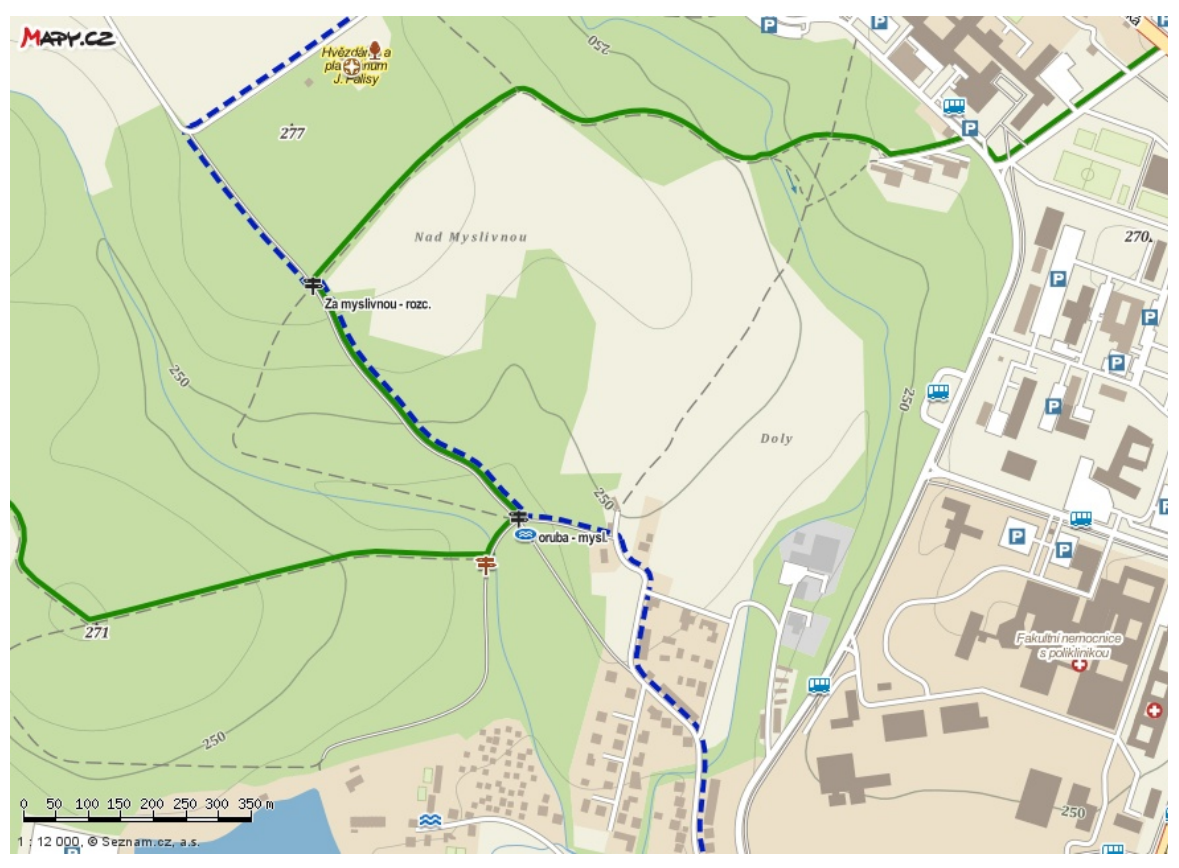

\section{Example 73}

Find contour lines (contour plot) of the graph of the function $z=\frac{5 x}{x^{2}+y^{2}+1}$.

We substitute $z=k$, where $k \in \mathbb{R}$.

1. For $k=0$ (intersection of the graph with $x y$-plane) we get $0=\frac{5 x}{x^{2}+y^{2}+1} \Rightarrow x=0$, the contour line is precisely $y$-axis.

2. For $k \neq 0$ we get

$$
\begin{aligned}
k=\frac{5 x}{x^{2}+y^{2}+1} & \Rightarrow x^{2}+y^{2}+1=\frac{5 x}{k} \Rightarrow x^{2}-\frac{5 x}{k}+y^{2}+1=0 \\
& \Rightarrow\left(x-\frac{5}{2 k}\right)^{2}+y^{2}=\frac{25}{4 k^{2}}-1
\end{aligned}
$$


The contour lines are circles but only in the case, where right-hand side of the equation is positive,

$$
\begin{aligned}
\frac{25}{4 k^{2}}-1>0 & \Rightarrow \frac{25}{4 k^{2}}>1 \Rightarrow 25>4 k^{2} \Rightarrow k^{2}<\frac{25}{4} \Rightarrow|k|<\frac{5}{2} \\
& \Rightarrow k \in\left(-\frac{5}{2}, 0\right) \cup\left(0, \frac{5}{2}\right) .
\end{aligned}
$$

3. For $k= \pm \frac{5}{2}$ holds $(x \mp 1)^{2}+y^{2}=0$. We get as contours two singular circles, points $[1,0]$ and $[-1,0]$.
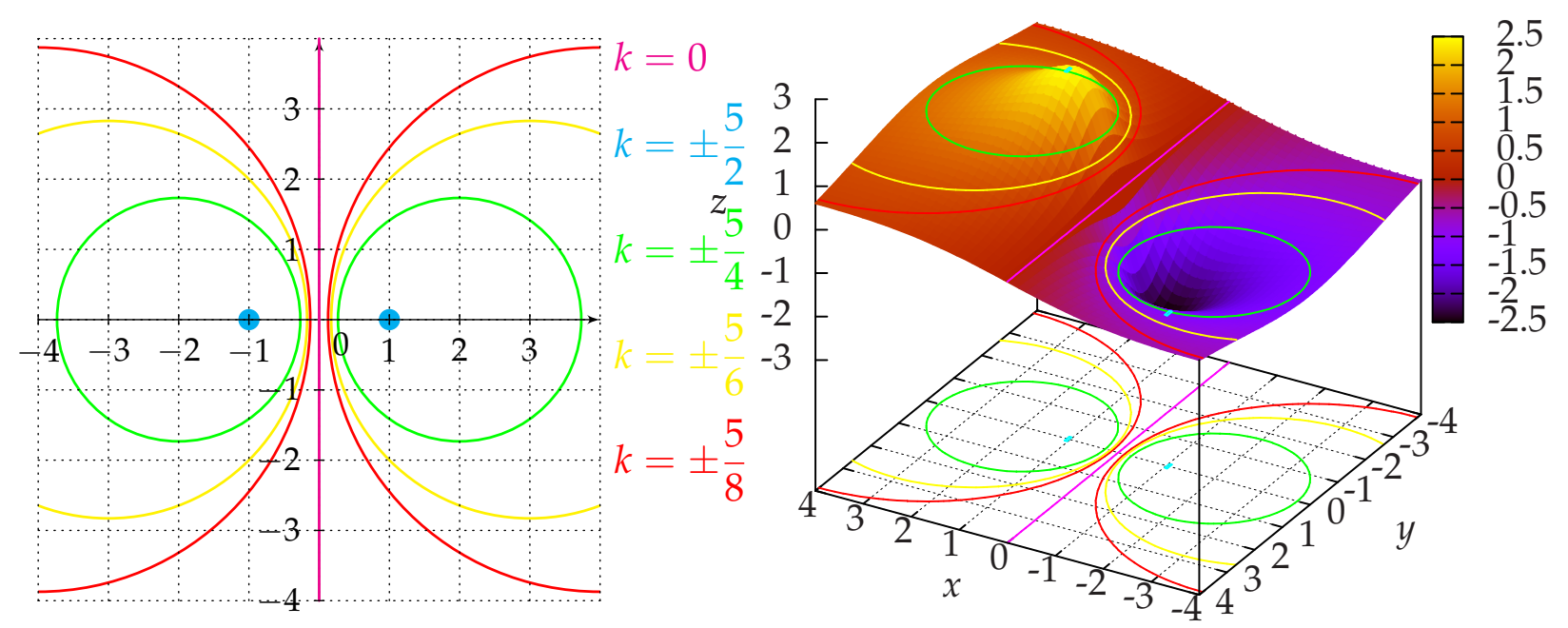

Exercise 74

Find contour lines of the graph of the functions
a) $z=x^{2}+y^{2}-4$
b) $z=\frac{2 y}{x^{2}}+1$
c) $z=x^{2} y^{2}$

\subsection{Limits, continuity}

Limits and continuity of functions of two real variables are defined just like in the case of functions depending on one real variable.

\section{Definition}

We say that a function $z=f(x, y)$ has in a cluster point (accumulation point) $P=\left[x_{0}, y_{0}\right]$ limit $a \in \mathbb{R}$, if for every $\epsilon>0$ exists $\delta>0$ such that for every $X \in \stackrel{\circ}{\mathcal{O}}_{\delta}(P)$ holds $|f(X)-a|<\epsilon$.

The notion neighbourhood of a point is slightly generalized. In the case of functions depending on one real variable, it is an open interval. In our situation, functions depending on two real variables, it is an open circle (circle without boundary).

The neighbourhood $\stackrel{\circ}{\mathcal{O}}_{\delta}(P)$ is a set called punctured neighbourhood at the point $P$, it is an open circle with the center at $P$, radius is equal to $\delta$ and $P$ does not belong to this set. 


\section{Definition}

Let $U \subset \mathbb{R}^{2}$, a point $P \in \mathbb{R}^{2}$ is called cluster point of the set $U$, if its every punctured neighbourhood $\stackrel{\circ}{\mathcal{O}}(P)$ has non empty intersection with $U, \stackrel{\circ}{\mathcal{O}}(P) \cap U \neq \varnothing$.

On the following figure, the points $X$ and $Y$ are cluster points of the set $U$. The point $Z$ in not cluster point of $U$.

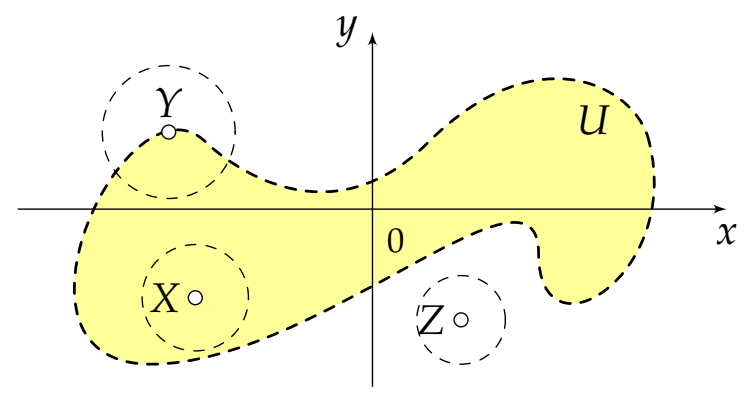

In the case of functions depending on one real variable, if one wants to calculate the limit at a point, it is enough to study behaviour of a function along left respective right part of appropriate neighbourhood. In our situation, the neighbourhood is an open circle, thus we have infinite possible options how to tend to a given limit point.

In general, limits of functions depending on two real variables can be solved by direct substitution of the limit point into the given function. There is a different task, try to proof that the limit does not exist.

Notation:

$$
\lim _{X \rightarrow P} f(X)=a, \quad \lim _{[x, y] \rightarrow\left[x_{0}, y_{0}\right]} f(x, y)=a .
$$

\section{Definition}

We say that a function $z=f(x, y)$ is continuous at the point $P=\left[x_{0}, y_{0}\right] \in D_{f}$ if it holds

$$
\lim _{[x, y] \rightarrow\left[x_{0}, y_{0}\right]} f(x, y)=f\left(x_{0}, y_{0}\right) .
$$

A function is continuous if it is continuous at every point belonging to its domain.

A function is continuous at a point if there exists a limit at this point which is equal to the function value at this point.

\section{Example 75}

Find the limit of the function $z=\frac{y(x+1)}{x^{3}+1}$ at the point $[-1,0]$ and proof that the limit of the function $z=\frac{x^{2}+x}{x y+y}$ does not exist at the point $[0,0]$. 
First part of the task,

$$
\lim _{[x, y] \rightarrow[-1,0]} \frac{y x+y}{x^{3}+1}={ }^{\prime}{ }^{0} 0 "=\lim _{[x, y] \rightarrow[-1,0]} \frac{y(x+1)}{(x+1)\left(x^{2}-x+1\right)}=\lim _{[x, y] \rightarrow[-1,0]} \frac{y}{x^{2}-x+1}=0 .
$$

In the second part of the task we need to prove that the limit does not exist. The limit does not exist if its value depends on the choice of the way of approaching or changes for different ways of approaching to a limit point. However, if the limit does not depend on the particular choice of way of approaching, then that does not mean that the limit does exist.

Let us try to tend to the limit point along lines passing the origin of the coordinate system, $y=k x, k \in \mathbb{R}$. We substitute $y=k x$ in the function $z=\frac{x^{2}+x}{x y+y}$,

$$
\lim _{[x, y] \rightarrow[0,0]} \frac{x^{2}+x}{x y+y}={ }^{\prime} \frac{0}{0} \stackrel{y=k x}{=} \lim _{x \rightarrow 0} \frac{x^{2}+x}{k x^{2}+k x}=\lim _{x \rightarrow 0} \frac{x^{2}+x}{k\left(x^{2}+x\right)}=\lim _{x \rightarrow 0} \frac{1}{k},
$$

the limit depends on the parameter $k$, for different values of $k$ the limit is different, i.e. the limit of the function $z=\frac{x^{2}+x}{x y+y}$ at the point $[0,0]$ does not exist.
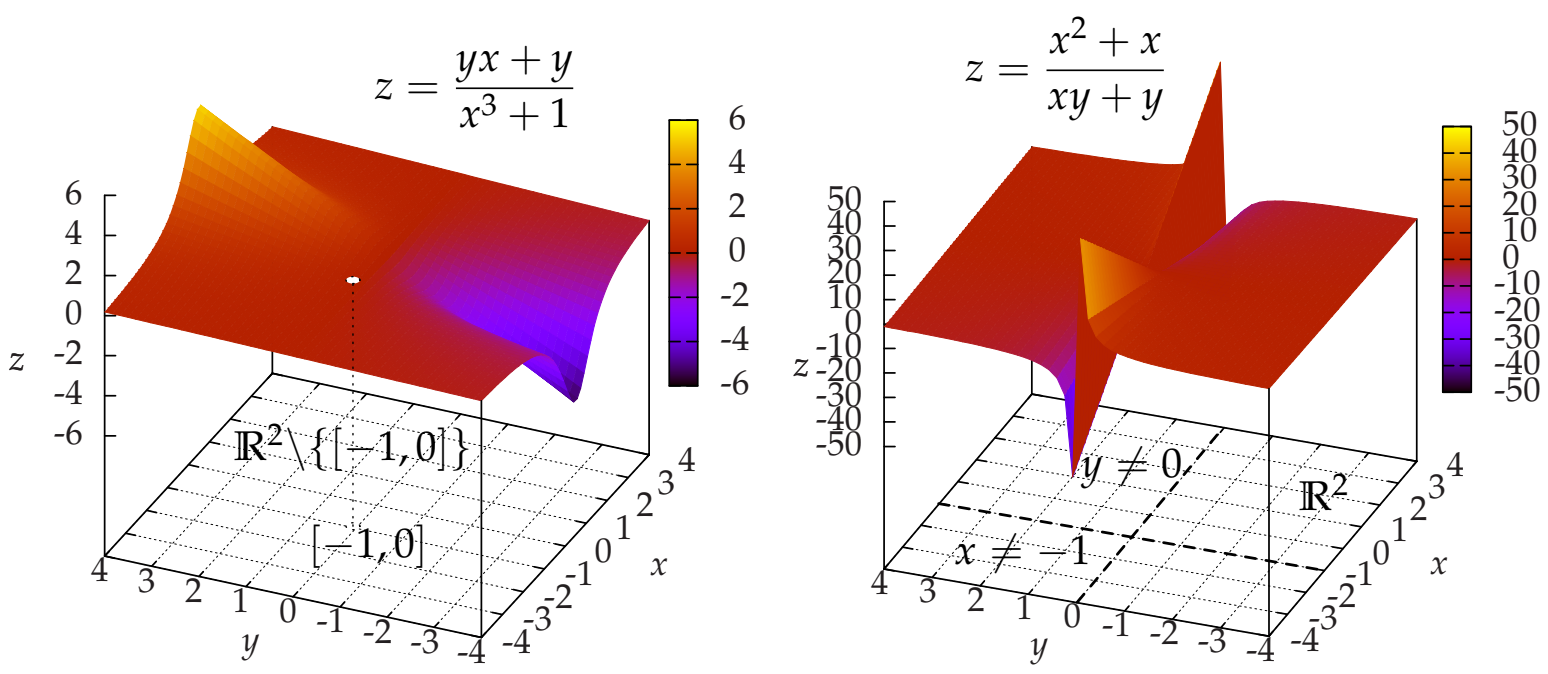

Exercise 76

Solve,
a) $\lim _{[x, y] \rightarrow[1,2]} \frac{x y+x-y}{x^{3}-2}$
b) $\lim _{[x, y] \rightarrow\left[\frac{\pi}{2},-\frac{5 \pi}{6}\right]} \sin (2 x+y)$ 


\subsection{Partial derivatives}

\section{Definition}

We say that a function $z=f(x, y)$ has partial derivative with respect to $x$ (of the first order) at the point $A=\left[x_{0}, y_{0}\right]$, if there exists proper limit

$$
\frac{\partial f}{\partial x}(A)=\lim _{h \rightarrow 0} \frac{f\left(x_{0}+h, y_{0}\right)-f\left(x_{0}, y_{0}\right)}{h}
$$

Analogously, one can define partial derivative with respect to $y$,

$$
\frac{\partial f}{\partial y}(A)=\lim _{h \rightarrow 0} \frac{f\left(x_{0}, y_{0}+h\right)-f\left(x_{0}, y_{0}\right)}{h} .
$$

Remark

- Notation: $\frac{\partial f}{\partial x}(A), \frac{\partial z}{\partial x}(A), f_{x}(A), f_{x}^{\prime}(A)$, etc.

- Partial derivatives $\frac{\partial f}{\partial x}$ respective $\frac{\partial f}{\partial y}$ are again functions depending on two real variables.

- Partial derivatives of functions depending on three and more real variables can be defined analogously.

- When we calculate partial derivative with respect to $x$, we keep $y$ fixed and treat it as a constant, i.e. such function is differentiated like the function depending only on one real variable. For partial derivatives with respect to $y$ we proceed in the same way, we keep $x$ fixed.

In fact, the calculation of partial derivatives of functions depending on two real variable reduces to the calculation of derivatives of functions depending on one real variable. One can use the same formulas and rules for differentiation of functions depending on one real variable.

\section{Theorem}

Let there exist partial derivatives of functions $f(x, y)$ and $g(x, y)$ with respect to $x=x_{1}$ and $y=x_{2}$ on $Q \subseteq D_{f} \cap D_{g}$ at the point $X$. Then for every $i=1,2$ holds,

$$
\begin{aligned}
\frac{\partial}{\partial x_{i}}(f \pm g)(X) & =\frac{\partial f}{\partial x_{i}}(X) \pm \frac{\partial g}{\partial x_{i}}(X) \\
\frac{\partial}{\partial x_{i}}(f \cdot g)(X) & =\frac{\partial f}{\partial x_{i}}(X) \cdot g(X)+f(X) \cdot \frac{\partial g}{\partial x_{i}}(X) \\
\frac{\partial}{\partial x_{i}}\left(\frac{f}{g}\right)(X) & =\frac{\frac{\partial f}{\partial x_{i}}(X) \cdot g(X)-f(X) \cdot \frac{\partial g}{\partial x_{i}}(X)}{g^{2}(X)} .
\end{aligned}
$$




\section{- Definition}

Partial derivatives of the second order of a function $z=f(x, y)$ are defined as follows,

$$
\frac{\partial^{2} f}{\partial x^{2}}=\frac{\partial}{\partial x}\left(\frac{\partial f}{\partial x}\right), \frac{\partial^{2} f}{\partial y^{2}}=\frac{\partial}{\partial y}\left(\frac{\partial f}{\partial y}\right), \frac{\partial^{2} f}{\partial x \partial y}=\frac{\partial}{\partial y}\left(\frac{\partial f}{\partial x}\right), \frac{\partial^{2} f}{\partial y \partial x}=\frac{\partial}{\partial x}\left(\frac{\partial f}{\partial y}\right)
$$

The following Schwarz theorem describes important property of mixed partial derivatives.

\section{Theorem (Schwarz)}

If mixed partial derivatives $\frac{\partial^{2} f}{\partial x \partial y}, \frac{\partial^{2} f}{\partial y \partial x}$ are continuous at the point $A=\left[x_{0}, y_{0}\right]$, then they are at this point equal, $\frac{\partial^{2} f}{\partial x \partial y}(A)=\frac{\partial^{2} f}{\partial y \partial x}(A)$.

\subsection{Geometrical meaning of partial derivatives}

Geometrical meaning of partial derivatives is the same as in the case of the derivatives of functions of one real variable. It is the slope of a tangent line at the given point.

The plane $\sigma: y=y_{0}$ is parallel with $x z$-plane ( $x z$-plane is expressed by $y=0$ ). The intersection of the plane $\sigma$ with the graph of the function $z=f(x, y)$ is the curve $\kappa$. Partial derivative $\frac{\partial f}{\partial x}(A), A=\left[x_{0}, y_{0}\right]$, is a slope $(\tan \alpha)$ of the tangent line $t_{\mathcal{K}}$ to the curve $\kappa$ at the point $A=\left[x_{0}, y_{0}, z_{0}=f\left(x_{0}, y_{0}\right)\right]$.

The plane $v: x=x_{0}$ is parallel with $y z$-plane ( $y z$-plane is expressed by $x=0$ ). The intersection of the plane $v$ with the graph of the function $z=f(x, y)$ is the curve $\lambda$. Partial derivative $\frac{\partial f}{\partial y}(A), A=\left[x_{0}, y_{0}\right]$, is a slope $(\tan \beta)$ of the tangent line $t_{\lambda}$ to the curve $\lambda$ at the point $\boldsymbol{A}=\left[x_{0}, y_{0}, z_{0}=f\left(x_{0}, y_{0}\right)\right]$.
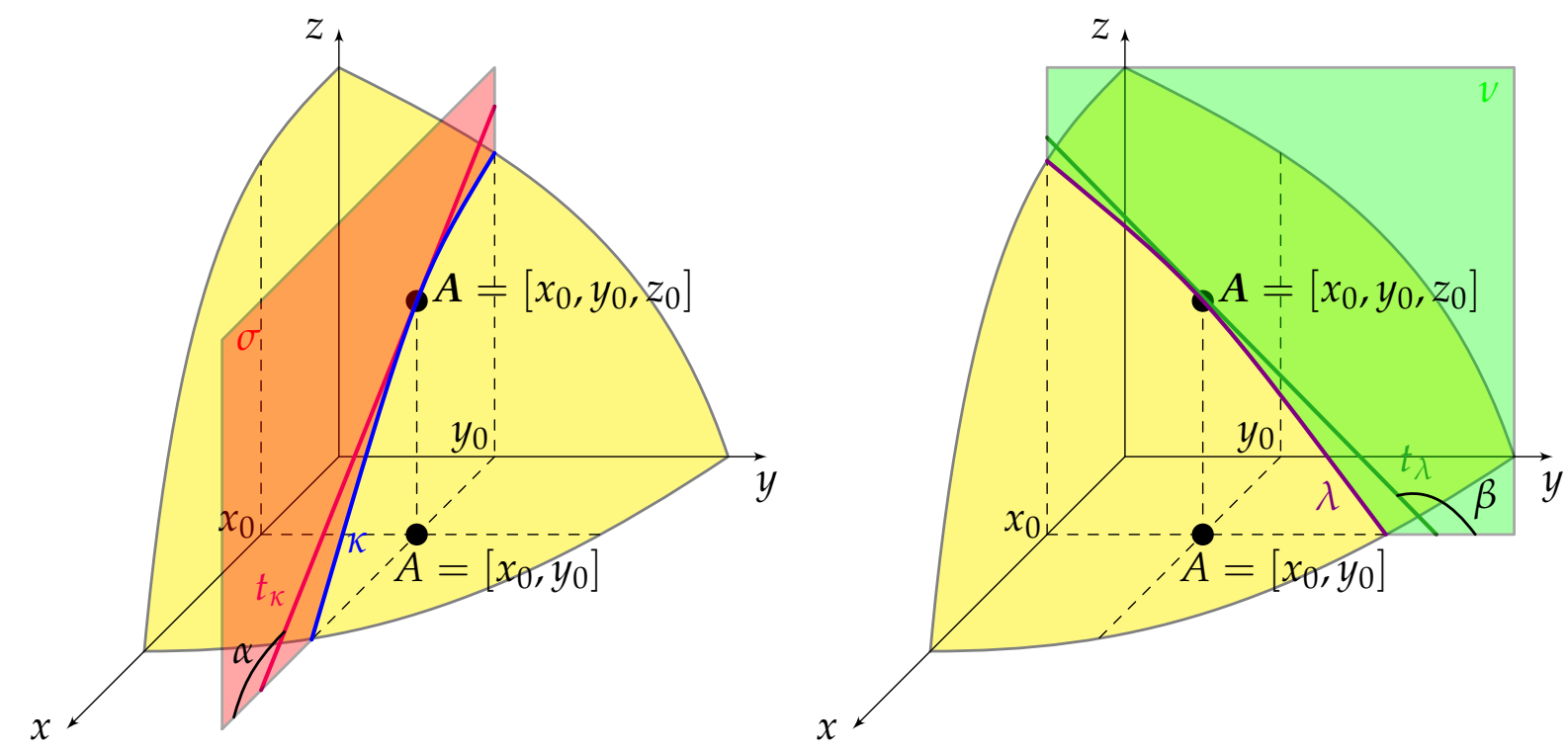


\section{Example 77}

Find partial derivatives of the first order of the function $z=x^{2} \sin ^{2}\left(x y^{2}\right)$ at the point $[\pi, 0]$.

We calculate partial derivatives with respect to all independent variables, i.e. with respect to $x$ and $y$. If we fix one independent variable, e.g. $y$, such variable is considered as a constant $c$, the function $z=f(x, y)$ becomes the function depending on one real variable $z=f(x, c)$, and can be easily differentiated.

Partial derivative $\frac{\partial z}{\partial x}$ of the function $z$ with respect to $x$ :

because the function $z$ is differentiated with respect to $x$, the variable $y$ is considered as a constant. Our task is to differentiate multiplication of two functions depending on $x$, the function $x^{2}$ and the composed function $\sin ^{2}\left(x y^{2}\right)$, which must be differentiated in the following order of components: second power, sine function, $x y^{2}$; in the expression $x y^{2}$ is $y^{2}$ a constant factor, therefore we have to differentiate only $x$,

$$
\begin{aligned}
\frac{\partial z}{\partial x} & =\frac{\partial}{\partial x}\left(x^{2}\right) \cdot \sin ^{2}\left(x y^{2}\right)+x^{2} \cdot \frac{\partial}{\partial x}\left(\sin ^{2}\left(x y^{2}\right)\right) \\
& =2 x \sin ^{2}\left(x y^{2}\right)+x^{2} \cdot 2 \sin \left(x y^{2}\right) \cdot \cos \left(x y^{2}\right) \cdot y^{2}=2 x \sin ^{2}\left(x y^{2}\right)+2 x^{2} y^{2} \sin \left(x y^{2}\right) \cos \left(x y^{2}\right) .
\end{aligned}
$$

Partial derivative $\frac{\partial z}{\partial y}$ of the function $z$ with respect to $y$ :

differentiation is analogous, as a constant we mean $x$ variable, again we differentiate a composed function; $x^{2}$ is a constant factor, i.e. only second factor must be differentiated with respect to $y$ as a composed function with the following order of components: second root, sine function, $x y^{2}$; in the expression $x y^{2}$ is $x$ considered as a constant factor, thus we differentiate only $y^{2}$,

$$
\frac{\partial z}{\partial y}=x^{2} \cdot \frac{\partial z}{\partial y}\left(\sin ^{2}\left(x y^{2}\right)\right)=x^{2} \cdot 2 \sin \left(x y^{2}\right) \cdot \cos \left(x y^{2}\right) \cdot x 2 y=4 x^{3} y \sin \left(x y^{2}\right) \cos \left(x y^{2}\right) .
$$

Partial derivatives are again functions depending on two real variables and can be easily evaluated at the given point by direct substitution:

$$
\frac{\partial z}{\partial x}(\pi, 0)=0, \quad \frac{\partial z}{\partial y}(\pi, 0)=0
$$

Exercise 78

Find partial derivatives of the first order:
a) $z=x^{2}+y^{2}$
c) $z=\left(x^{3}+1\right) y(x+1)$
e) $z=\frac{\sqrt{x y}}{\ln \left(x-y^{2}\right)}$
b) $z=\sin (2 x+y)$
d) $z=\frac{x y+x-y}{x^{3}-2}$
f) $z=\tan (\ln (x y))$ 


\section{Exercise 79}

Find partial derivatives of the first order:
a) $z=\frac{x}{x^{2}+y^{2}}$ at $[1,-1]$
b) $z=\ln \arctan \frac{x}{y-x}$ at $[1,2]$

Example 80

Find partial derivatives of the second order of the function $z=x^{y}$ at the point $[1,1]$.

At first, we need to calculate partial derivatives of the first order,

In the derivative with respect to $x$, we differentiate power function

$$
\frac{\partial z}{\partial x}=y x^{y-1}
$$

In the derivative with respect to $y$, we differentiate exponential function $\frac{\partial z}{\partial y}=x^{y} \ln x$

To find partial derivatives of the second order, it is necessary to differentiate partial derivatives of the first order with respect to $x$ and $y$,

$$
\begin{gathered}
\frac{\partial^{2} z}{\partial x^{2}}=\frac{\partial}{\partial x}\left(\frac{\partial z}{\partial x}\right)=\frac{\partial}{\partial x}\left(y x^{y-1}\right)=y(y-1) x^{y-2} \\
\frac{\partial^{2} z}{\partial x \partial y}=\frac{\partial}{\partial y}\left(\frac{\partial z}{\partial x}\right)=\frac{\partial}{\partial y}\left(y x^{y-1}\right)=x^{y-1}+y x^{y-1} \ln x \\
\frac{\partial^{2} z}{\partial y \partial x}=\frac{\partial}{\partial x}\left(\frac{\partial z}{\partial y}\right)=\frac{\partial}{\partial x}\left(x^{y} \ln x\right)=y x^{y-1} \ln x+x^{y} \frac{1}{x} \\
\frac{\partial^{2} z}{\partial y^{2}}=\frac{\partial}{\partial y}\left(\frac{\partial z}{\partial y}\right)=\frac{\partial}{\partial y}\left(x^{y} \ln x\right)=x^{y} \ln x \ln x=x^{y} \ln ^{2} x
\end{gathered}
$$

Stress that in the case of continuous functions with continuous partial derivatives, the mixed derivatives are the same according to Schwarz theorem. Indeed:

$$
\frac{\partial^{2} z}{\partial y \partial x}=y x^{y-1} \ln x+x^{y} \frac{1}{x}=y x^{y-1} \ln x+x^{y} x^{-1}=y x^{y-1} \ln x+x^{y-1}=x^{y-1}+y x^{y-1} \ln x=\frac{\partial^{2} z}{\partial x \partial y} .
$$

Finally, the simple evaluation of partial derivatives of the second order at the point $[1,1]$ remains,

$$
\frac{\partial^{2} z}{\partial x^{2}}(1,1)=0, \quad \frac{\partial^{2} z}{\partial x \partial y}(1,1)=1, \quad \frac{\partial^{2} z}{\partial y \partial x}(1,1)=1, \quad \frac{\partial^{2} z}{\partial y^{2}}(1,1)=0
$$

\section{Exercise 81}

Find partial derivatives of the second order:
a) $z=\cot (x+2 y)$
b) $z=x \mathrm{e}^{(y+1)}$
c) $z=x^{y}$ 


\section{Exercise 82}

Find partial derivatives of the second order:
a) $z=x^{2} \ln y$ at $[3,1]$
b) $z=y \mathrm{e}^{-x y^{2}}$ at $[-1,1]$

\section{Example 83}

Find partial derivative of the fourth order $\frac{\partial^{4} z}{\partial x^{2} \partial y^{2}}$ of the function $z=2 x^{2} \mathrm{e}^{y}+\sin \left(x y^{2}\right)$.

We differentiate the function $z$ according to single variables. We start differentiation with respect to $x$, then again with respect to $x$ and finally two times with respect to $y$.

$$
\begin{aligned}
\frac{\partial z}{\partial x} & =4 x \mathrm{e}^{y}+\cos \left(x y^{2}\right) \cdot y^{2}=4 x \mathrm{e}^{y}+y^{2} \cos \left(x y^{2}\right) \\
\frac{\partial^{2} z}{\partial x^{2}} & =4 \mathrm{e}^{y}+y^{2}\left(-\sin \left(x y^{2}\right) \cdot y^{2}\right)=4 \mathrm{e}^{y}-y^{4} \sin \left(x y^{2}\right) \\
\frac{\partial^{3} z}{\partial x^{2} \partial y} & =4 \mathrm{e}^{y}-\left[4 y^{3} \sin \left(x y^{2}\right)+y^{4} \cos \left(x y^{2}\right) \cdot x 2 y\right]=4 \mathrm{e}^{y}-4 y^{3} \sin \left(x y^{2}\right)-2 x y^{5} \cos \left(x y^{2}\right) \\
\frac{\partial^{4} z}{\partial x^{2} \partial y^{2}} & =4 \mathrm{e}^{y}-\left[12 y^{2} \sin \left(x y^{2}\right)+4 y^{3} \cos \left(x y^{2}\right) \cdot x 2 y\right]-\left[10 x y^{4} \cos \left(x y^{2}\right)+2 x y^{5}\left(-\sin \left(x y^{2}\right) \cdot x 2 y\right)\right] \\
& =4 \mathrm{e}^{y}-12 y^{2} \sin \left(x y^{2}\right)-8 x y^{4} \cos \left(x y^{2}\right)-10 x y^{4} \cos \left(x y^{2}\right)+4 x^{2} y^{6} \sin \left(x y^{2}\right) \\
& =4 \mathrm{e}^{y}-12 y^{2} \sin \left(x y^{2}\right)-18 x y^{4} \cos \left(x y^{2}\right)+4 x^{2} y^{6} \sin \left(x y^{2}\right)
\end{aligned}
$$

\section{Exercise 84}

Find partial derivative of the fourth order $\frac{\partial^{4} f}{\partial x^{2} \partial y^{2}}$ of the function $z=\ln (2 x+y)$.

\subsection{Differentials}

\section{Definition}

We say that a function $z=f(x, y)$ is differentiable at the point $A=\left[x_{0}, y_{0}\right]$, or it has differential at a point, if its increment $\Delta z$ on the neighbourhood of the point $A$ can be expressed as

$$
\Delta z=f\left(x_{0}+h, y_{0}+k\right)-f\left(x_{0}, y_{0}\right)=\mathcal{A} h+\mathcal{B} k+\rho \cdot \tau(h, k),
$$

where $\mathcal{A}$ and $\mathcal{B}$ are constants, $\rho=\sqrt{h^{2}+k^{2}}$ and $\lim _{[h, k] \rightarrow[0,0]} \tau(h, k)=0$. The function $z=f(x, y)$ is called differentiable, if it is differentiable at every point belonging to its domain. 


\section{Theorem}

If a function $z=f(x, y)$ is differentiable at the point $A$, then there exist partial derivatives of the first order at the point $A$ and it holds

$$
\mathcal{A}=\frac{\partial f}{\partial x}(A), \quad \mathcal{B}=\frac{\partial f}{\partial y}(A) .
$$

\section{Remark}

The number $h$ represents an increment on $x$-axis, $k$ is an increment on $y$-axis and we usually denote those increments by $h=\mathrm{d} x$ respective $k=\mathrm{d} y$. For the increment on $z$-axis at point $A$ with known values of $\mathrm{d} x$ and $\mathrm{d} y$ we get

$$
\Delta z=\frac{\partial f}{\partial x}(A) \mathrm{d} x+\frac{\partial f}{\partial y}(A) \mathrm{d} y+\rho \cdot \tau(\mathrm{d} x, \mathrm{~d} y) .
$$

\section{Definition}

If a function $z=f(x, y)$ is differentiable, the expression

$$
\mathrm{d} z=\mathrm{d} f(x, y)=\frac{\partial f}{\partial x} \mathrm{~d} x+\frac{\partial f}{\partial y} \mathrm{~d} y
$$

is called differential of the function $z=f(x, y)$.

\section{Theorem}

If a function $z=f(x, y)$ is differentiable at the point $A$, then it is continuous at this point.

\section{Theorem}

If partial derivatives of the first order of a function $z=f(x, y)$ are continuous at $A$, then the function $z=f(x, y)$ is differentiable (and thus continuous) at the point $A$.

\subsection{Geometrical meaning of differential, formulas}

Differential of the function $z=f(x, y)$ at the point $A$ with respect to known increments $\mathrm{d} x$ and $\mathrm{d} y$ is an increment on the tangent plane to the graph of the function $f$ at the point $A$. 


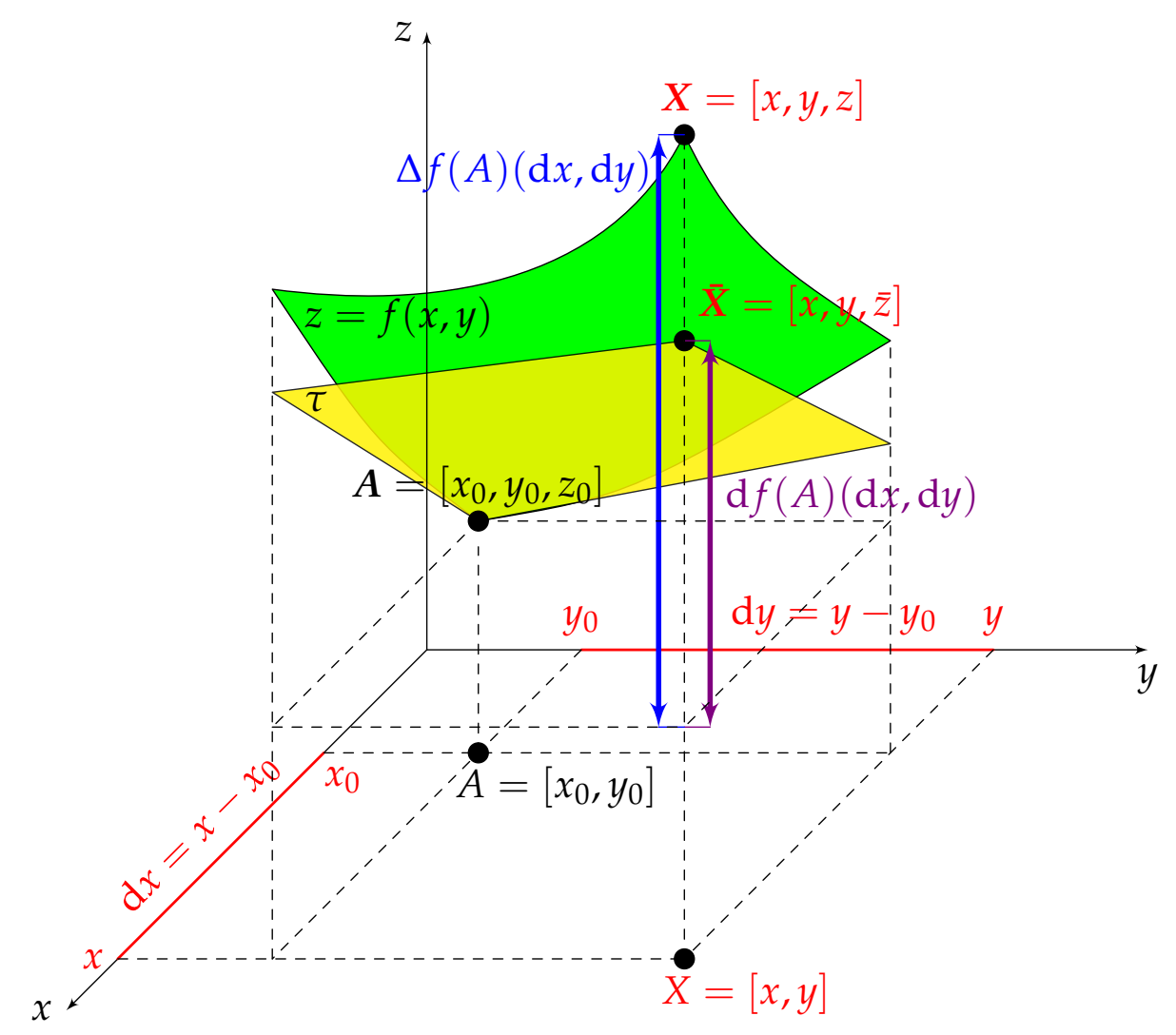

- Remark

- Differential of the function $z=f(x, y)$

$$
\mathrm{d} z=\frac{\partial f}{\partial x} \mathrm{~d} x+\frac{\partial f}{\partial y} \mathrm{~d} y
$$

- Differential of the function $z$ at the point $A=\left[x_{0}, y_{0}\right]$

$$
\mathrm{d} z(A)=\frac{\partial f}{\partial x}(A) \cdot\left(x-x_{0}\right)+\frac{\partial f}{\partial y}(A) \cdot\left(y-y_{0}\right) \text {. }
$$

- Differential of the function $z$ at the point $A=\left[x_{0}, y_{0}\right]$ with respect to known increments $\mathrm{d} x, \mathrm{~d} y$,

$$
\mathrm{d} z(A)(\mathrm{d} x, \mathrm{~d} y)=\frac{\partial f}{\partial x}(A) \cdot \mathrm{d} x+\frac{\partial f}{\partial y}(A) \cdot \mathrm{d} y \in \mathbb{R}
$$

- Differential of the second order of the function $z=f(x, y)$

$$
\mathrm{d}^{2} z=\frac{\partial^{2} f}{\partial x^{2}} \mathrm{~d} x^{2}+2 \frac{\partial^{2} f}{\partial x \partial y} \mathrm{~d} x \mathrm{~d} y+\frac{\partial^{2} f}{\partial y^{2}} \mathrm{~d} y^{2}
$$

- Approximate calculation of function values

$$
f(x, y) \approx f\left(x_{0}, y_{0}\right)+\mathrm{d} f\left(x_{0}, y_{0}\right)(\mathrm{d} x, \mathrm{~d} y) .
$$




\section{Example 85}

Find differential of the function $z=f(x, y)=\sqrt{x y}$ at the point $A=[2,1]$. Calculate approximately the function value $f(2.04,0.99)$.

At first, we calculate partial derivatives of the first order with respect to $x$ and $y$,

$$
\frac{\partial f}{\partial x}=\frac{y}{2 \sqrt{x y}}, \quad \frac{\partial f}{\partial y}=\frac{x}{2 \sqrt{x y}} .
$$

For differential $\mathrm{d} z$ of the function $z$ we have,

$$
\mathrm{d} z=\frac{y}{2 \sqrt{x y}} \mathrm{~d} x+\frac{x}{2 \sqrt{x y}} \mathrm{~d} y=\frac{1}{2 \sqrt{x y}}(y \mathrm{~d} x+x \mathrm{~d} y) .
$$

We evaluate differential $\mathrm{d} z$ at the point $A$,

$$
\mathrm{d} z(A)=\mathrm{d} z(2,1)=\frac{1}{2 \sqrt{2}}(1(x-2)+2(y-1))=\frac{\sqrt{2}}{4}(x+2 y-4) .
$$

Note that differential at a point is a linear function depending on two real variables. For approximate calculation of function values we need to find out increments from the point $A=[2,1]$ to the point $[2.04,0.99]$,

$$
\mathrm{d} x=x-x_{0}=2.04-2=0.04 ; \quad \mathrm{d} y=y-y_{0}=0.99-1=-0.01 .
$$

We calculate the function value $f(A)=f(2,1)=\sqrt{2}$ and the value of differential

$\mathrm{d} z(A)(\mathrm{d} x, \mathrm{~d} y)=\mathrm{d} z(2,1)(0.04,-0.01)=\frac{1}{2 \sqrt{2}}(1 \cdot 0.04-2 \cdot 0.01)=\frac{1}{2 \sqrt{2}} 0.02=\frac{1}{2 \sqrt{2}} \frac{2}{100}=\frac{\sqrt{2}}{200}$.

The approximate function value $f(2.04,0.99)$ is

$$
f(2.04,0.99) \approx f(A)+\mathrm{d} f(A)(\mathrm{d} x, \mathrm{~d} y)=\sqrt{2}+\frac{\sqrt{2}}{200}=\sqrt{2}\left(1+\frac{1}{200}\right) .
$$

Exercise 86

Find differentials of the first order
a) $z=\tan \left(x^{2}+y^{2}\right)$
b) $z=\frac{\sqrt{x}}{\log (x+2 y)}$
c) $z=\left(x^{3}+y^{3}\right) \sin (x y)$

\section{Exercise 87}

Find differentials of the first order at the point
a) $z=\mathrm{e}^{x^{2} y^{2}-4}$ at $[-1,2]$
b) $z=\arcsin \frac{y}{x+1}$ at $[1,1]$ 


\section{Exercise 88}

Find approximate function value of the function $z=\sqrt[3]{x+y}$ at $[4.08,3.99]$.

\section{Example 89}

Find differential of the second order of the function $z=f(x, y)=\frac{x+y}{x-y}$.

We calculate partial derivatives with respect to $x$ and $y$,

$$
\begin{aligned}
& \frac{\partial f}{\partial x}=\frac{1 \cdot(x-y)-(x+y) \cdot 1}{(x-y)^{2}}=\frac{-2 y}{(x-y)^{2}}, \\
& \frac{\partial f}{\partial y}=\frac{1 \cdot(x-y)-(x+y) \cdot(-1)}{(x-y)^{2}}=\frac{2 x}{(x-y)^{2}} .
\end{aligned}
$$

We find out partial derivatives of the second order,

$$
\begin{gathered}
\frac{\partial^{2} f}{\partial x^{2}}=\frac{\partial}{\partial x}\left(\frac{\partial f}{\partial x}\right)=\frac{\partial}{\partial x}\left(-2 y(x-y)^{-2}\right)=-2 y(-2)(x-y)^{-3} \cdot 1=\frac{4 y}{(x-y)^{3}}, \\
\frac{\partial^{2} f}{\partial x \partial y}=\frac{\partial}{\partial y}\left(\frac{\partial f}{\partial x}\right)=\frac{\partial}{\partial y}\left(\frac{-2 y}{(x-y)^{2}}\right)=\frac{-2(x-y)^{2}-(-2 y) 2(x-y) \cdot(-1)}{(x-y)^{4}}=\frac{-2(x+y)}{(x-y)^{3}} \\
\frac{\partial^{2} f}{\partial y \partial x}=\frac{\partial}{\partial x}\left(\frac{\partial f}{\partial y}\right)=\frac{\partial}{\partial x}\left(\frac{2 x}{(x-y)^{2}}\right)=\frac{2(x-y)^{2}-2 x \cdot 2(x-y) \cdot 1}{(x-y)^{4}}=\frac{-2(x+y)}{(x-y)^{3}} \\
\frac{\partial^{2} f}{\partial y^{2}}=\frac{\partial}{\partial y}\left(\frac{\partial f}{\partial y}\right)=\frac{\partial}{\partial y}\left(2 x(x-y)^{-2}\right)=2 x(-2)(x-y)^{-3} \cdot(-1)=\frac{4 x}{(x-y)^{3}} .
\end{gathered}
$$

For differential of the second order we have $\mathrm{d}^{2} z=\frac{4 y}{(x-y)^{3}} \mathrm{~d} x^{2}-4 \frac{x+y}{(x-y)^{3}} \mathrm{~d} x \mathrm{~d} y+\frac{4 x}{(x-y)^{3}} \mathrm{~d} y^{2}=\frac{4}{(x-y)^{3}}\left(y \mathrm{~d} x^{2}-(x+y) \mathrm{d} x \mathrm{~d} y+x \mathrm{~d} y^{2}\right)$.

\section{Exercise 90}

Find differentials of the second order
a) $z=\frac{x y}{x+y}$
b) $z=\sin (5 x+2 y)$

\subsection{Tangent plane, normal line, Taylor polynomial}

Theorem

Let a function $z=f(x, y)$ be differentiable at the point $A=\left[x_{0}, y_{0}\right]$. Then, at the point $A=\left[x_{0}, y_{0}, z_{0}=f\left(x_{0}, y_{0}\right)\right]$ there exists a tangent plane to the graph of the function $z=f(x, y)$ given by the equation

$$
\tau: z-z_{0}=\frac{\partial f}{\partial x}(A)\left(x-x_{0}\right)+\frac{\partial f}{\partial y}(A)\left(y-y_{0}\right) .
$$


The line $n$ perpendicular to the tangent plane and passing the point $A$ is called normal to the graph of the function $z=f(x, y)$. Its direction vector is collinear with the normal vector of the tangent plane, $\vec{s}_{n}=\vec{n}=\left(\frac{\partial f}{\partial x}(A), \frac{\partial f}{\partial y}(A),-1\right)$.

\section{Theorem}

The normal line to the graph of a function $z=f(x, y)$ at the point $A$ is determined by the parametric equations

$$
n: \quad x=x_{0}+\frac{\partial f}{\partial x}(A) t, \quad y=y_{0}+\frac{\partial f}{\partial y}(A) t, \quad z=z_{0}-t, \quad t \in \mathbb{R} .
$$

\section{Theorem}

Let a function $z=f(x, y)$ be at least $(m+1)$-times continuously differentiable on the neighbourhood $A \in D_{f}$. Then, at the point $X \in \mathcal{O}(A)$ holds

$$
\begin{aligned}
f(X) & =f(A)+\frac{\mathrm{d} f(A)}{1 !}+\frac{\mathrm{d}^{2} f(A)}{2 !}+\cdots+\frac{\mathrm{d}^{m} f(A)}{m !}+R_{m}, \text { where } \\
R_{m} & =\frac{\mathrm{d}^{m+1} f(A+\kappa(X-A))}{(m+1) !}, \kappa \in(0,1) .
\end{aligned}
$$

\section{Definition}

The expression from the preceding theorem is called Taylor expansion of the function $f$ on the neighbourhood of the point $A$. The value $R_{m}$ is called Lagrange remainder of Taylor polynomial. The polynomial

$$
T_{m}(X)=f(A)+\frac{\mathrm{d} f(A)}{1 !}+\frac{\mathrm{d}^{2} f(A)}{2 !}+\cdots+\frac{\mathrm{d}^{m} f(A)}{m !}
$$

is called Taylor polynomial of $m$-th order of the function $f$ at the point $A$. If $A=[0,0]$, we talk about Maclaurin polynomial.

\section{Example 91}

Find the equation of the tangent plane and normal line to the graph of the function $z=f(x, y)=\sqrt{2 x}-\sqrt{3 y}-x$ at the point $A=[2,3, ?]$.

The point $A$ is a tangent point, $x_{0}=2, y_{0}=3$, we calculate its $z$ component, $z_{0}=$ $f\left(x_{0}, y_{0}\right)=f(2,3)=-3$.

We calculate partial derivatives of the function $f$,

$$
\frac{\partial f}{\partial x}=\frac{\sqrt{2}}{2} \frac{1}{\sqrt{x}}-1, \quad \frac{\partial f}{\partial y}=-\frac{\sqrt{3}}{2} \frac{1}{\sqrt{y}}
$$

at the point $A=[2,3]$,

$$
\frac{\partial f}{\partial x}(A)=-\frac{1}{2}, \quad \frac{\partial f}{\partial y}=-\frac{1}{2}
$$


The equation of the tangent plane is

$$
\tau: z+3=-\frac{1}{2}(x-2)-\frac{1}{2}(y-3)
$$

or in general form

$$
\tau: x+y+2 z+1=0 \text {. }
$$

For parametric equations of the normal line we get

$$
\begin{aligned}
x & =2-\frac{1}{2} t \\
n: \quad y & =3-\frac{1}{2} t, \quad t \in \mathbb{R} \\
z & =-3-t
\end{aligned}
$$

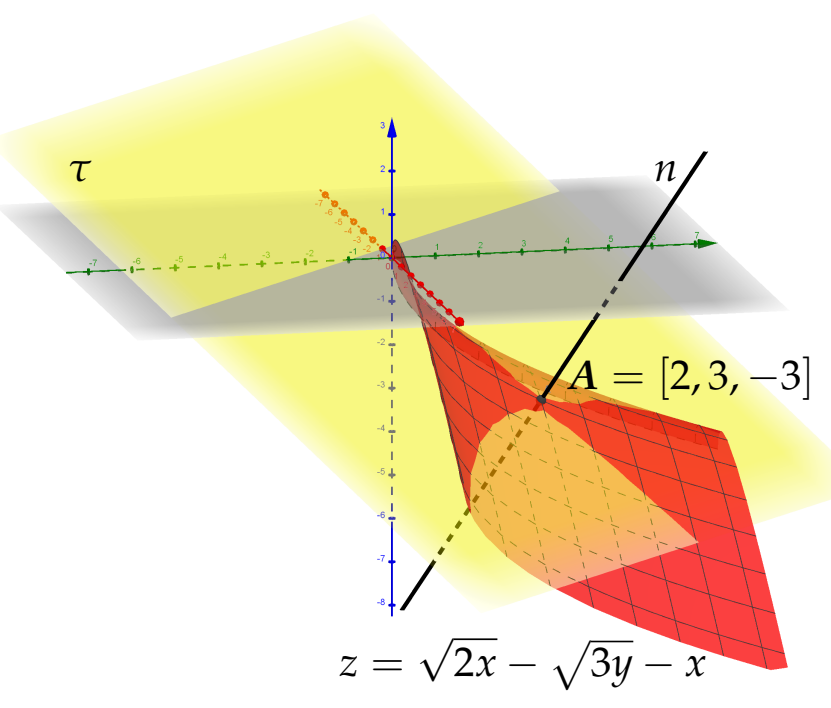

Exercise 92

Find the equation of the tangent plane $\tau$ and normal line $n$ to the graph of the function

a) $z=\ln \left(x^{2}-3 y\right)$ at the point $A=[2,1, ?]$,

b) $z=\sqrt{x^{2}+x y+1}$ at the point $A=[0,4, ?]$.

\section{Example 93}

Find Taylor polynomial of the second order of the function $z=f(x, y)=2 x^{2}-x y+$ $3 y^{2}+x-y+1$ at the point $A=[1,1]$.

We calculate function values $f(A), \mathrm{d} f(A)$ and $\mathrm{d}^{2} f(A)$. Then we use the formula for Taylor polynomial of the second order at the point $A=\left[x_{0}, y_{0}\right]=[1,1]$. 


$$
\begin{aligned}
f(A) & =5 \\
\mathrm{~d} f(A) & =\left.(4 x-y+1)\right|_{[1,1]}(x-1)+\left.(-x+6 y-1)\right|_{[1,1]}(y-1) \\
& =4(x-1)+4(y-1) \\
& =4 x+4 y-8 \\
\mathrm{~d}^{2} f(A) & =\left.4\right|_{[1,1]}(x-1)^{2}+\left.2 \cdot(-1)\right|_{[1,1]}(x-1)(y-1)+\left.6\right|_{[1,1]}(y-1)^{2} \\
& =4 x^{2}-8 x+4-2 x y+2 x+2 y-2+6 y^{2}-12 y+6 \\
& =4 x^{2}-2 x y+6 y^{2}-6 x-10 y+8 \\
T_{2}(A) & =5+\frac{4 x+4 y-8}{1 !}+\frac{4 x^{2}-2 x y+6 y^{2}-6 x-10 y+8}{2 !} \\
& =5+4 x+4 y-8+2 x^{2}-x y+3 y^{2}-3 x-5 y+4 \\
& =2 x^{2}-x y+3 y^{2}+x-y+1
\end{aligned}
$$

Exercise 94

Find the second order Taylor polynomial of the function
a) $z=3 x^{2} y+4 x y^{2}+x^{3}$ at the point $A=[2,-1]$,
b) $z=\ln \frac{1}{x y}$ at the point $A=[-2,-3]$.

\subsection{Implicit functions}

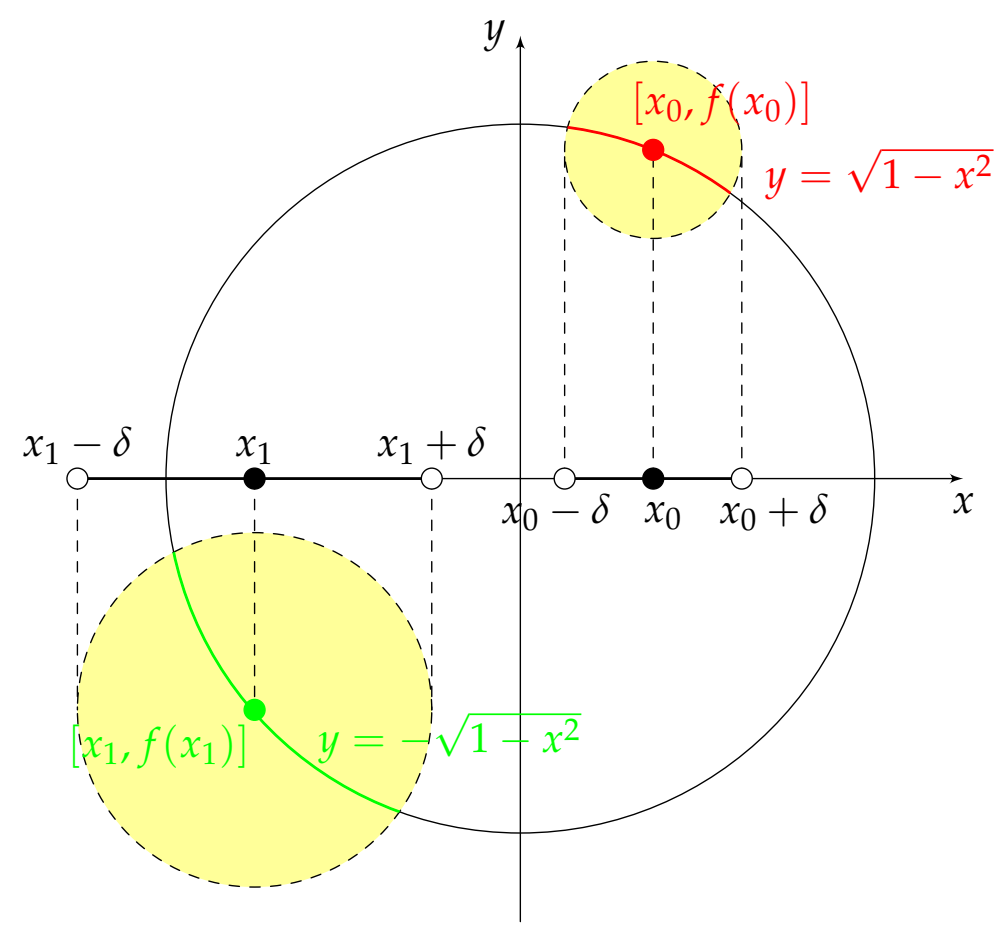




\section{Definition}

Let a function $z=F(x, y)$ be a function depending on two real variables. We consider the curve

$$
M=\left\{[x, y] \in D_{F} \mid F(x, y)=0\right\} .
$$

Let $A=\left[x_{0}, y_{0}\right] \in M$ be a point, $\mathcal{O}_{\delta}(A) \subset \mathbb{R}^{2}$ is its neighbourhood, $\delta>0$. If the equation $F(x, y)=0$ on the neighbourhood of $A$ determines the function $y=f(x)$ such that it holds

$$
F(x, f(x))=0, \quad \forall[x, f(x)] \in \mathcal{O}_{\delta}(A),
$$

we say that the function $f$ is on neighbourhood of $A$ defined implicitly by the equation $F(x, y)=0$.

On the figure one can see a circle with radius equaled to 1 and with the center placed at the origin of the Cartesian coordinate system,

$$
x^{2}+y^{2}-1=0 \Rightarrow y^{2}=1-x^{2} \Rightarrow|y|=\sqrt{1-x^{2}}, x \in[-1,1] .
$$

On the interval $(-1,1)$ there are determined two implicit functions by the preceding equation, $y=\sqrt{1-x^{2}}$ (upper half-circle) and $y=-\sqrt{1-x^{2}}$ (lower half-circle). At the point $[1,0]$ and $[-1,0]$ the implicit function does not exist, every neighbourhood of such points contains points lying on both upper and lower half-circles.

\section{Remark}

Not every equation $F(x, y)=0$ represents unique implicit function.

\section{Theorem}

Let a function $z=F(x, y)$ be continuous on the neighbourhood of the point $A=\left[x_{0}, y_{0}\right]$ and $F(A)=0$. Let $F$ has continuous partial derivative $\frac{\partial F}{\partial y}(A)$ at the point $A$ and holds $\frac{\partial F}{\partial y}(A) \neq 0$. Then there exist a neighbourhood of the point $A$ such that by the equation $F(x, y)=0$ is determined unique continuous implicit function defined on this neighbourhood.
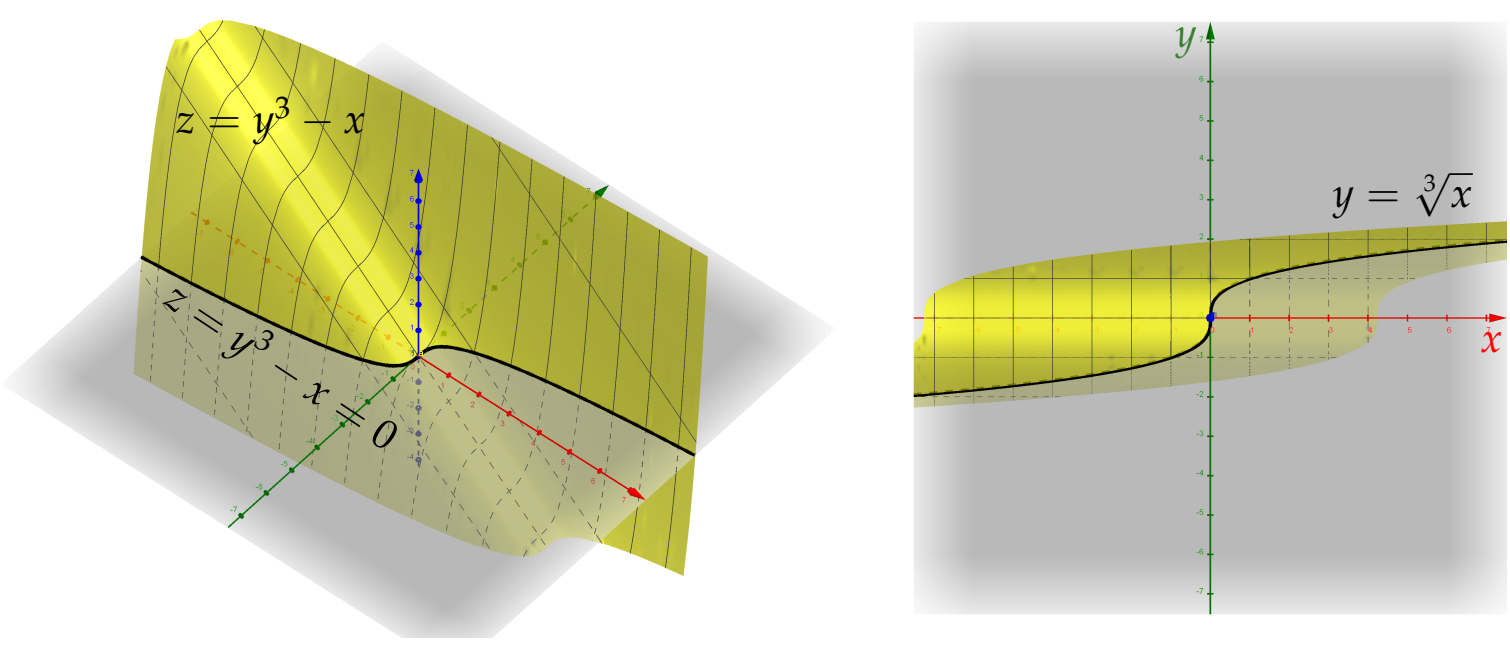


\section{Remark}

The condition that the partial derivative of the function $F$ with respect to $y$ is not null is only a sufficient condition for the existence of an implicit function. For example, from the equation $y^{3}-x=0$ follows $F(x, y)=y^{3}-x$ and at the point $[0,0]$ it holds $\frac{\partial F}{\partial y}(0,0)=$ $\left.3 y^{2}\right|_{[0,0]}=0$. Nevertheless, there exists the only one implicit function $y=\sqrt[3]{x}$ on the neighbourhood of the point $[0,0]$, see figure on the left.

\section{Theorem}

Let the assumptions of the preceding theorem be fulfilled. Let exist continuous partial derivatives of the function $F$. The implicit function, which is given by the equation $F(x, y)=0$ on the neighbourhood of the point $A$, has the derivative $f^{\prime}$ at the point $x_{0}$ and it holds

$$
f^{\prime}\left(x_{0}\right)=-\frac{\frac{\partial F}{\partial x}(A)}{\frac{\partial F}{\partial y}(A)} .
$$

\section{Theorem}

Tangent or tangent line $t$ respective normal or normal line $n$ to the implicit function $y=$ $f(x)$ given by the equation $F(x, y)=0$ at the point $A$ are determined by the following equations,

$$
\begin{aligned}
& t: \frac{\partial F}{\partial x}(A)\left(x-x_{0}\right)+\frac{\partial F}{\partial y}(A)\left(y-y_{0}\right)=0 \\
& n: \frac{\partial F}{\partial y}(A)\left(x-x_{0}\right)-\frac{\partial F}{\partial x}(A)\left(y-y_{0}\right)=0
\end{aligned}
$$

\section{Example 95}

Find the derivative of the implicit function determined by the equation $x^{3}+y+y^{2}-$ $2 x y=3$ at the point $A=[1,-1]$.

We have

$$
F(x, y)=x^{3}+y+y^{2}-2 x y-3
$$

We find out partial derivatives

$$
\frac{\partial F}{\partial x}=3 x^{2}-2 y, \quad \frac{\partial F}{\partial y}=1+2 y-2 x
$$

The partial derivatives are continuous functions, moreover

$$
\frac{\partial F}{\partial y}(A)=1+2 y-\left.2 x\right|_{A=[1,-1]}=-3 \neq 0 .
$$


The derivative of an implicit function does exist and its the only one. We substitute into the formula for derivative of an implicit function at the point $A=\left[x_{0}, y_{0}\right]=[1,-1]$,

$$
f^{\prime}(1)=-\frac{3 x^{2}-\left.2 y\right|_{[1,-1]}}{1+2 y-\left.2 x\right|_{[1,-1]}}=-\frac{5}{-3}=\frac{5}{3} .
$$

The alternative method is based on the assumption, that in the equation $F(x, y)=0$ the dependence of $y$ on $x$ is assumed, $y=y(x)$, i.e. in this equation only one independent variable remains. Thus, the equation is differentiated with respect to $x$, both sides simultaneously,

$$
\frac{\mathrm{d}}{\mathrm{d} x}\left(x^{3}+y+y^{2}-2 x y-3=0\right) \Rightarrow 3 x^{2}+y^{\prime}+2 y y^{\prime}-2 y-2 x y^{\prime}=0 .
$$

From the equation we express $y^{\prime}$

$$
\begin{aligned}
y^{\prime}+2 y y^{\prime}-2 x y^{\prime}=-3 x^{2}+2 y & \Rightarrow y^{\prime}(1+2 y-2 x)=-3 x^{2}+2 y \\
& \Rightarrow \quad y^{\prime}=\frac{-3 x^{2}+2 y}{1+2 y-2 x}=-\frac{3 x^{2}-2 y}{1+2 y-2 x}
\end{aligned}
$$

Exercise 96

Using both methods find the derivative of the implicit function given by the equation
a) $x^{2}+y^{2}+y^{3}-x y=2$,
b) $\cot (3 y)=x^{2} y$.

\section{Example 97}

Find the tangent and normal line to the graph of the implicit function $y=f(x)$ given by the equation $\mathrm{e}^{x y}=x+2 y$ at the point $A=[1,0]$.

The function $F$ is given by

$$
F(x, y)=\mathrm{e}^{x y}-x-2 y .
$$

We find out partial derivatives of the function $F$ at the point $A=\left[x_{0}, y_{0}\right]=[1,0]$,

$$
\begin{aligned}
& \frac{\partial F}{\partial x}(A)=y \mathrm{e}^{x y}-\left.1\right|_{[1,0]}=-1 \\
& \frac{\partial F}{\partial y}(A)=x \mathrm{e}^{x y}-\left.2\right|_{[1,0]}=-1 .
\end{aligned}
$$

We construct the equation of the tangent $t$ to the graph of the implicit function,

$$
t:-(x-1)-y=0 \Rightarrow y=-x+1 \text {. }
$$

We construct the equation of the normal $n$ to the graph of the implicit function,

$$
n:-(x-1)+y=0 \Rightarrow y=x-1 \text {. }
$$




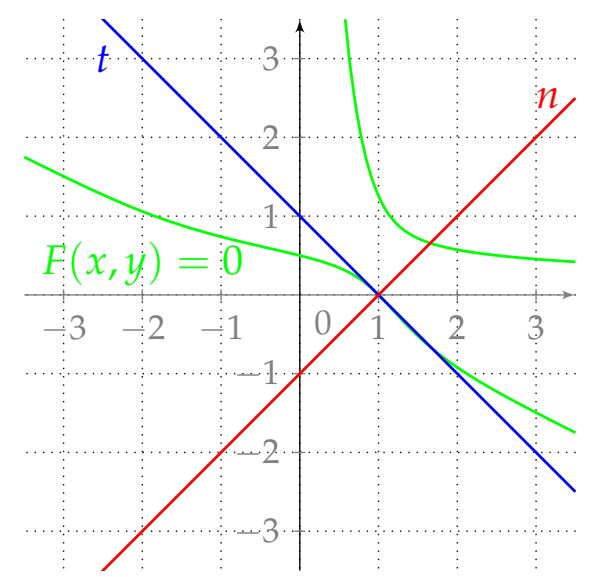

Exercise 98

Find the tangent and normal line to the implicit function $y=f(x)$ given by $\frac{x+y}{x-y}=2$ at the point $A=[3,1]$.

\subsection{Local extrema}

\section{Definition}

We say that a function $z=f(x, y)$ has local maximum at the point $A \in D_{f}$ if there exists a neighbourhood $\mathcal{O}(A) \subseteq D_{f}$ of the point $A$ such that $\forall X \in \mathcal{O}(A)$ holds $f(X) \leq f(A)$. If $f(X) \geq f(A)$ is satisfied $\forall X \in \mathcal{O}(A)$ then it is local minimum at the point $A$. In the case of strict inequalities we talk about strict local maxima or minima.

\section{Definition}

We say that the point $A \in D_{f}$ is stationary or critical point of a function $f(x, y)$ if

$$
\frac{\partial f}{\partial x}(A)=0, \quad \frac{\partial f}{\partial y}(A)=0
$$

\section{Fermat theorem - necessary condition for existence of an extremum}

- Theorem (Fermat)

Let a function $f$ has local extremum at the point $A$ and let there exist all partial derivatives of the first order at the point $A$. Then the point $A$ is a critical point of the function $f$.

- Remark

- Fermat theorem does not exclude the possibility that the function $f$ has an extremum at a point which is not the critical point of the function $f$ because some of its partial derivatives does not exist.

- The condition for critical points is equivalent with the condition $\mathrm{d} f(A)=0$. Nevertheless, if $\mathrm{d} f(A) \neq 0$, then a local extremum does not exist at the point $A$. 


\section{Theorem}

Let there exist at least continuous partial derivatives of the second order of a function $f$ at the critical point $A$, then

- $\mathrm{d}^{2} f(A)<0$, the function $f$ has strict local maximum at $A$,

- $\mathrm{d}^{2} f(A)>0$, the function $f$ has strict local maximum at $A$.

\section{Sufficient condition for the existence of an extremum}

\section{Theorem}

Let the function $f$ be twice continuously differentiable on a neighbourhood of the point $A$. Let $A$ be a critical point. If

$$
D_{2}=\frac{\partial^{2} f}{\partial x^{2}}(A) \frac{\partial^{2} f}{\partial y^{2}}(A)-\left(\frac{\partial^{2} f}{\partial x \partial y}(A)\right)^{2}>0,
$$

then the function $f$ has a strict local extremum at the point $A$. Moreover, if

$$
\begin{aligned}
& -D_{1}=\frac{\partial^{2} f}{\partial x^{2}}(A)<0, \text { the function } f \text { has a strict local maximum at } A, \\
& \text { - } D_{1}=\frac{\partial^{2} f}{\partial x^{2}}(A)>0, \text { the function } f \text { has a strict local minimum at } A .
\end{aligned}
$$

\section{Remark}

If $D_{2}=0$, nothing can be said about the existence of an extremum. This obstacle can be resolved in some cases by examining local properties of the function $f$ at the point $A$.

The number $D_{2}$ is determinant of the matrix $Q(A)$,

$$
Q(A)=\left(\begin{array}{ll}
\frac{\partial^{2} f}{\partial x^{2}}(A) & \frac{\partial^{2} f}{\partial x \partial y}(A) \\
\frac{\partial^{2} f}{\partial y \partial x}(A) & \frac{\partial^{2} f}{\partial y^{2}}(A)
\end{array}\right)
$$

\section{Example 99}

Find local extrema of the function $z=\mathrm{e}^{-x^{2}-y^{2}}\left(2 y^{2}+x^{2}\right)$.

The domain of the function $z$ is the whole set $\mathbb{R}^{2}$. We find partial derivatives of the first order and create the system of equations for critical points,

$$
\begin{aligned}
& \frac{\partial f}{\partial x}=\mathrm{e}^{-x^{2}-y^{2}}(-2 x)\left(2 y^{2}+x^{2}\right)+\mathrm{e}^{-x^{2}-y^{2}} 2 x=-2 x \mathrm{e}^{-x^{2}-y^{2}}\left(2 y^{2}+x^{2}-1\right)=0, \\
& \frac{\partial f}{\partial y}=\mathrm{e}^{-x^{2}-y^{2}}(-2 y)\left(2 y^{2}+x^{2}\right)+\mathrm{e}^{-x^{2}-y^{2}} 4 y=-2 y \mathrm{e}^{-x^{2}-y^{2}}\left(2 y^{2}+x^{2}-2\right)=0 .
\end{aligned}
$$


The exponential function $\mathrm{e}^{-x^{2}-y^{2}}$ is positive, thus

$$
\begin{aligned}
& x\left(2 y^{2}+x^{2}-1\right)=0 \\
& y\left(2 y^{2}+x^{2}-2\right)=0 .
\end{aligned}
$$

Suppose that $x=0$. From the second equation $y\left(2 y^{2}-2\right)=0$ we get the solutions $y=0$ and $y= \pm 1$. We got three critical points, $A_{1}=[0,0], A_{2}=[0,1]$ a $A_{3}=[0,-1]$.

In the second equation we suppose that $y=0$. From the first equation $x\left(x^{2}-1\right)=0$ we get the solutions $x=0$ and $x= \pm 1$. The point $[0,0]$ is already found, we have to add two new critical points $A_{4}=[1,0]$ and $A_{5}=[-1,0]$.

If $x \neq 0$ and $y \neq 0$, we need to solve the following system of equations,

$$
\begin{aligned}
& 2 y^{2}+x^{2}-1=0 \\
& 2 y^{2}+x^{2}-2=0 .
\end{aligned}
$$

If we subtract both equations we get $1=0$, i.e. the solution of the system does not exist. We found five different critical points:

$$
A_{1}=[0,0], \quad A_{2}=[0,1], \quad A_{3}=[0,-1], \quad A_{4}=[1,0], \quad A_{5}=[-1,0] .
$$

We continue the search for local extrema from the preceding worksheet. We found a total of five critical points. We create a matrix of the second order derivatives and we evaluate it at particular critical points $A_{i}, i=1,2,3,4,5$,

$$
\begin{gathered}
Q=\left(\begin{array}{cc}
\left(\left(-2+4 x^{2}\right)\left(2 y^{2}+x^{2}-1\right)-4 x^{2}\right) \mathrm{e}^{-x^{2}-y^{2}} & 4 x y \mathrm{e}^{-x^{2}-y^{2}}\left(2 y^{2}+x^{2}-3\right) \\
4 x y \mathrm{e}^{-x^{2}-y^{2}\left(2 y^{2}+x^{2}-3\right)} \quad\left(\left(-2+4 y^{2}\right)\left(2 y^{2}+x^{2}-2\right)-8 y^{2}\right) \mathrm{e}^{-x^{2}-y^{2}}
\end{array}\right), \\
Q\left(A_{1}\right)=\left(\begin{array}{ll}
2 & 0 \\
0 & 4
\end{array}\right), \quad Q\left(A_{2}\right)=\left(\begin{array}{cc}
-\frac{2}{\mathrm{e}} & 0 \\
0 & -\frac{8}{\mathrm{e}}
\end{array}\right), \quad Q\left(A_{3}\right)=\left(\begin{array}{cc}
-\frac{2}{\mathrm{e}} & 0 \\
0 & -\frac{8}{\mathrm{e}}
\end{array}\right), \\
Q\left(A_{4}\right)=\left(\begin{array}{cc}
-\frac{4}{\mathrm{e}} & 0 \\
0 & \frac{2}{\mathrm{e}}
\end{array}\right), \quad Q\left(A_{5}\right)=\left(\begin{array}{cc}
-\frac{4}{\mathrm{e}} & 0 \\
0 & \frac{2}{\mathrm{e}}
\end{array}\right) .
\end{gathered}
$$

The following table summarizes the classification of extrema at critical points:

\begin{tabular}{crrl}
\hline Critical point $A_{i}$ & $D_{1}$ & $D_{2}$ & extremum with the value $z=f\left(A_{i}\right)$ \\
\hline$A_{1}=[0,0]$ & $2>0$ & $8>0$ & strict local minimum $z=0$ \\
$A_{2}=[0,1]$ & $-\frac{2}{\mathrm{e}}<0$ & $\frac{16}{\mathrm{e}^{2}}>0$ & strict local maximum $z=\frac{2}{\mathrm{e}}$ \\
$A_{3}=[0,-1]$ & $-\frac{2}{\mathrm{e}}<0$ & $\frac{16}{\mathrm{e}^{2}}>0$ & strict local maximum $z=\frac{2}{\mathrm{e}}$ \\
$A_{4}=[1,0]$ & $-\frac{4}{\mathrm{e}}<0$ & $-\frac{8}{\mathrm{e}^{2}}<0$ & extremum does not exist \\
$A_{5}=[-1,0]$ & $-\frac{4}{\mathrm{e}}<0$ & $-\frac{8}{\mathrm{e}^{2}}<0$ & extremum does not exist \\
\hline
\end{tabular}




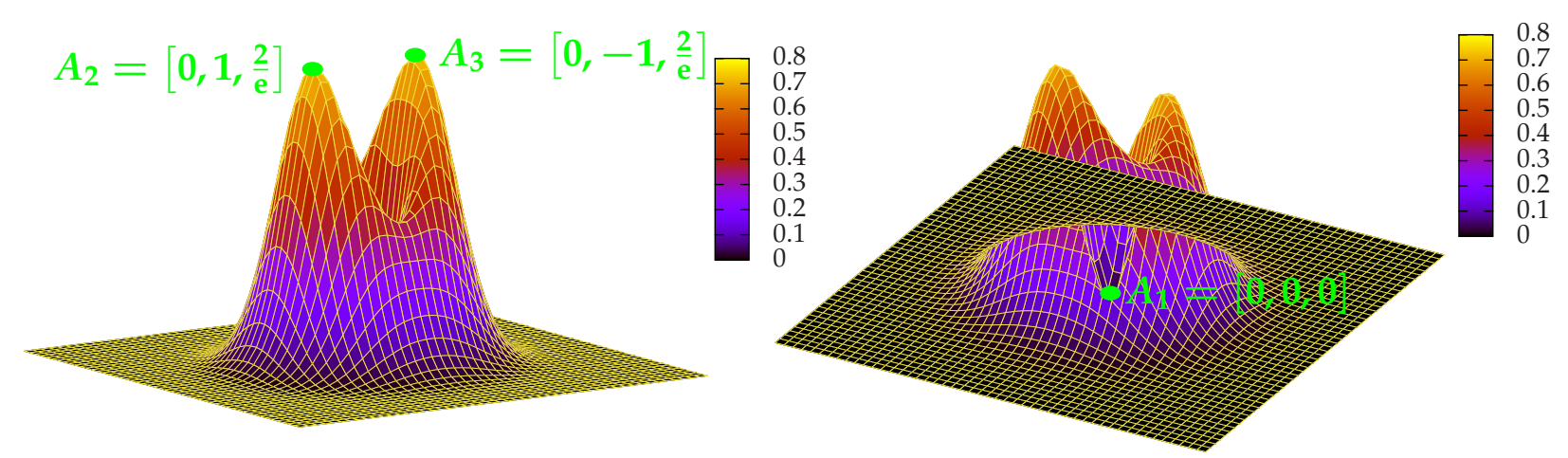

Exercise 100

Find local extrema of the function
a) $z=x^{2}+6 x+3 y^{2}-12 y+11$
c) $z=x^{2}-x y+3 x+y+3$
b) $z=3 x y-x+2 y$
d) $z=\left(x^{2}+4 x\right) y+y^{2}$

\subsection{Constraint extrema}

\section{Definition}

We say that a function $z=f(x, y)$ has constraint local extremum constrained by the condition $g(x, y)=0$ at the point $A=\left[x_{0}, y_{0}\right]$ if $\forall X \in \mathcal{O}(A) \subset D_{f}$ satisfying the condition $g(x, y)=0$ holds

- $f(X) \leq f(A)$, function $f$ has at $A$ constraint local maximum,

- $f(X) \geq f(A)$, function $f$ has at $A$ constraint local minimum.

\section{Geometrical interpretation of constraint extrema}

Constraint extremum can be found only among the points belonging to the domain of a function $f$ and lying on the curve $g(x, y)=0$. These points correspond to points on the surface $z=f(x, y)$ forming the spatial curve $\kappa$, the intersection curve of the surface with the cylinder generated by the equation $g(x, y)=0$. From the geometrical point of view it is a problem of finding local extrema of a spatial curve.

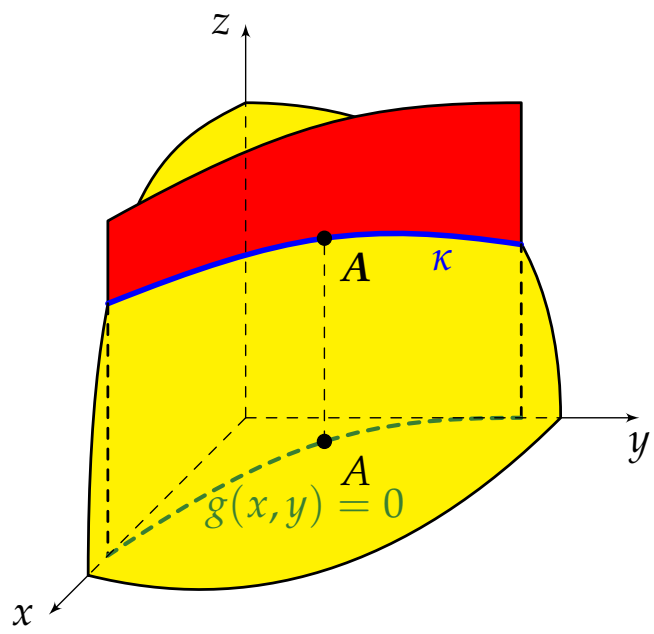




\section{Lagrange method}

Theorem (Lagrange)

Let a function $z=f(x, y)$ and a condition $g(x, y)=0$ be given. If the function

$$
\Phi(x, y, \lambda)=f(x, y)+\lambda g(x, y), \quad \lambda \in \mathbb{R},
$$

has a local extremum at its critical point, then the function $f$ has a constraint local extremum constrained by the condition $g(x, y)=0$ at this point.

- Remark

- The function $\Phi$ is called Lagrange function, the number $\lambda$ is called Lagrange multiplier.

- Critical points can be found as a solution of the following system of equations,

$$
\frac{\partial \Phi}{\partial x}=0, \quad \frac{\partial \Phi}{\partial y}=0, \quad g(x, y)=0
$$

- If one can uniquely express $y=\varphi(x)$ or $x=\psi(y)$ from the condition $g(x, y)=0$, then we search for constraint local extrema as local extrema of the function $z=$ $f(x, \varphi(x))$ or $z=f(\psi(y), y)$, i.e. of the functions depending on one real variable.

\section{Example 101}

Find constraint local extrema of $z=f(x, y)=\sqrt{4 x+y^{2}+5}$ with respect to the condition $2 x-3-y=0$.

We find domain of the function $z$,

$$
4 x+y^{2}+5 \geq 0 \Rightarrow x \geq-\frac{1}{4} y^{2}-\frac{5}{4}, \text { see the figure below. }
$$

From the equation of the constraint $2 x-3-y=0$ one can uniquely express both $y$ and $x$. Let express $y$,

$$
y=2 x-3
$$

We substitute the constraint in the function $z$,

$$
z=\sqrt{4 x+(2 x-3)^{2}+5}=\sqrt{4 x+4 x^{2}-12 x+9+5}=\sqrt{4 x^{2}-8 x+14},
$$

we get the function $z=z(x, \varphi(x))$ depending only on one variable and we see that $D_{z}=\mathbb{R}$. We calculate the first order derivative, we set this derivate equal to zero and finally obtain the equation for critical points of this function,

$$
z^{\prime}=\frac{\mathrm{d} z}{\mathrm{~d} x}=\frac{8 x-8}{2 \sqrt{4 x^{2}-8 x+14}}=\frac{4 x-4}{\sqrt{4 x^{2}-8 x+14}}=0 \quad \Rightarrow \quad 4 x-4=0 \Rightarrow x=1 .
$$

Domain of the first order derivative is the set $D_{z^{\prime}}=\mathbb{R}$. On the interval $(-\infty, 1)$ the derivative is negative, $z^{\prime}<0$, the function $z$ is a decreasing function on this interval. On the 
interval $(1, \infty)$ the first derivative is positive, $z^{\prime}>0$, i.e. the function $z$ is an increasing function on this interval. At the critical point $x=1$ the derivative changes its sign from to + , it means that at the point $x=1$ the function $z$ has a local minimum.

We substitute the point $x=1$ into the equation of the constraint, we get $y=-1$. It is easy to check that the point $A$ belongs to domain of the function $z=\sqrt{4 x+y^{2}+5}$, see figure below.

The function $z=\sqrt{4 x+y^{2}+5}$ has constraint local minimum $z=f(1,-1)=\sqrt{10}$ at the point $A=[1,-1]$.

Quite analogously we can proceed if we choose the unique expression of $x$ from the equation of the constraint at the beginning.

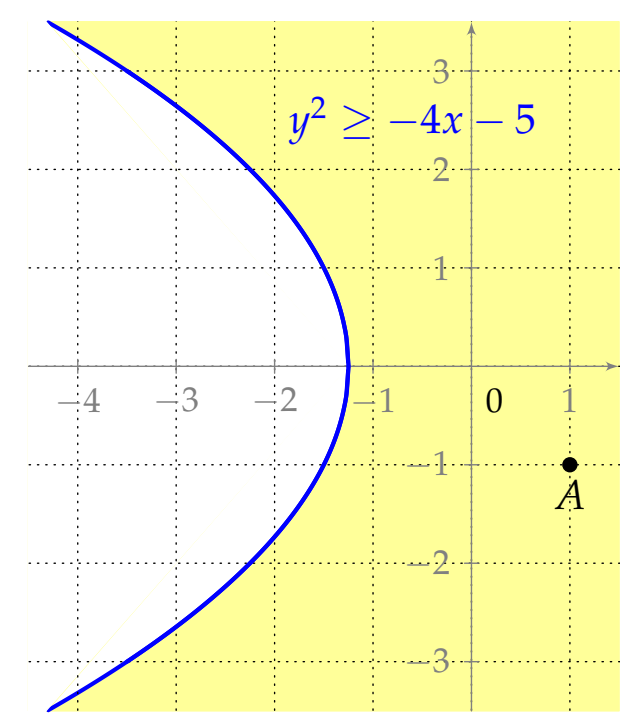

Example 102

Find constraint local extrema of $z=f(x, y)=-8 x+6 y-5$ with respect to the condition $x^{2}+y^{2}=100$.

We construct Lagrange function for $f(x)=-8 x+6 y-5$ and $g(x, y)=x^{2}+y^{2}-100$,

$$
\Phi(x, y, \lambda)=-8 x+6 y-5+\lambda\left(x^{2}+y^{2}-100\right) .
$$

We calculate first order partial derivatives of $\Phi$ and create equations for critical points of $\Phi$,

$$
\frac{\partial \Phi}{\partial x}=-8+2 \lambda x=0, \quad \frac{\partial \Phi}{\partial y}=6+2 \lambda y=0,
$$

we solve the following system

$$
-8+2 \lambda x=0 \Rightarrow x=\frac{4}{\lambda} \quad 6+2 \lambda y=0 \Rightarrow y=-\frac{3}{\lambda}
$$

we substitute into the constraint equation instead of $x$ and $y$,

$$
\begin{aligned}
x^{2}+y^{2}=100 & \Rightarrow\left(\frac{4}{\lambda}\right)^{2}+\left(-\frac{3}{\lambda}\right)^{2}=100 \Rightarrow \frac{16}{\lambda^{2}}+\frac{9}{\lambda^{2}}=100 \\
& \Rightarrow 100 \lambda^{2}=25 \Rightarrow \lambda^{2}=\frac{1}{4} \Rightarrow \lambda_{1,2}= \pm \frac{1}{2}
\end{aligned}
$$


We calculate critical points $A=[x, y]$ substituting $\lambda$,

$$
\lambda_{1}=\frac{1}{2} \Rightarrow A_{1}=[8,-6], \quad \lambda_{2}=-\frac{1}{2} \Rightarrow A_{2}=[-8,6] .
$$

We construct classification matrix and evaluate this matrix at critical points,

$$
Q=\left(\begin{array}{cc}
\frac{\partial^{2} \Phi}{\partial x^{2}} & \frac{\partial^{2} \Phi}{\partial x \partial y} \\
\frac{\partial^{2} \Phi}{\partial y \partial x} & \frac{\partial^{2} \Phi}{\partial y^{2}}
\end{array}\right)=\left(\begin{array}{cc}
2 \lambda & 0 \\
0 & 2 \lambda
\end{array}\right), \quad Q\left(A_{1}\right)=\left(\begin{array}{cc}
1 & 0 \\
0 & 1
\end{array}\right), \quad Q\left(A_{2}\right)=\left(\begin{array}{cc}
-1 & 0 \\
0 & -1
\end{array}\right)
$$

At $A_{1}=[8,-6]$ is $D_{1}>0, D_{2}>0$. The function $z=f(x, y)$ has constraint local minimum $z=-105$ at $A_{1}=[8,-6]$. At the point $A_{2}=[-8,6]$ is $D_{1}<0, D_{2}>0$. The function $z=f(x, y)$ has constraint local maximum $z=95$ at $A_{2}=[-8,6]$.

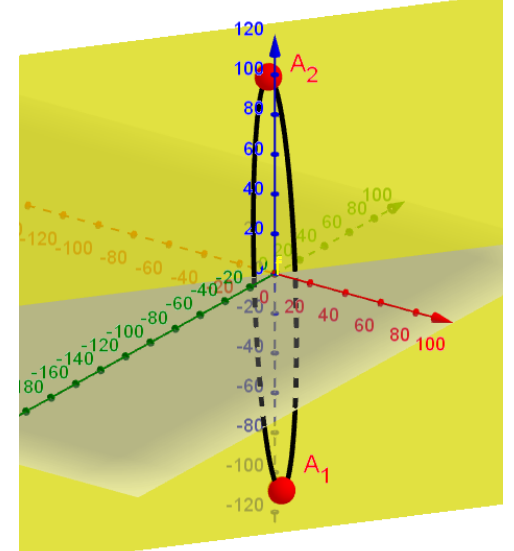

Example 103

Find constraint local extrema of $z=f(x, y)=x y-3 x^{2}+5$ with respect to the condition $2 x^{2}-y-12=0$.

We construct Lagrange function for $f(x)=x y-3 x^{2}+5$ and $g(x, y)=2 x^{2}-y-12$,

$$
\Phi(x, y, \lambda)=x y-3 x^{2}+5+\lambda\left(2 x^{2}-y-12\right) .
$$

We calculate first order partial derivatives of $\Phi$ and create equations for critical points of $\Phi$,

$$
\frac{\partial \Phi}{\partial x}=y-6 x+4 \lambda x=0, \quad \frac{\partial \Phi}{\partial y}=x-\lambda=0 \quad \Rightarrow \quad x=\lambda, \quad y=6 \lambda-4 \lambda^{2}
$$

We substitute into the constrain equation,

$$
2 x^{2}-y-12=0 \Rightarrow 2 \lambda^{2}-6 \lambda+4 \lambda^{2}-12=0 \Rightarrow \lambda^{2}-\lambda-2=0 \Rightarrow \lambda_{1}=-1, \lambda_{2}=2 \text {. }
$$

We calculate critical points $A=[x, y]$ substituting $\lambda$,

$$
\lambda_{1}=-1 \quad \Rightarrow \quad A_{1}=[-1,-10], \quad \lambda_{2}=2 \quad \Rightarrow \quad A_{2}=[2,-4] .
$$

We construct classification matrix and evaluate this matrix at critical points,

$$
Q=\left(\begin{array}{cc}
\frac{\partial^{2} \Phi}{\partial x^{2}} & \frac{\partial^{2} \Phi}{\partial x \partial y} \\
\frac{\partial^{2} \Phi}{\partial y \partial x} & \frac{\partial^{2} \Phi}{\partial y^{2}}
\end{array}\right)=\left(\begin{array}{cc}
4 \lambda-6 & 1 \\
1 & 0
\end{array}\right), \quad Q\left(A_{1}\right)=\left(\begin{array}{cc}
-10 & 1 \\
1 & 0
\end{array}\right), \quad Q\left(A_{2}\right)=\left(\begin{array}{ll}
2 & 1 \\
1 & 0
\end{array}\right)
$$


At $A_{1}=[-1,-10]$ and at $A_{2}=[2,-4]$ the determinant $D_{2}<0$. The Lagrange function $\Phi$ does not have extremum at these points. But, we can not say, that constraint extremum of the function $f(x, y)$ does not exist!

The constraint extrema can be found directly by substituting the constraint equation into the given function,

$z(x)=f\left(x, 2 x^{2}-12\right)=2 x^{3}-3 x^{2}-12 x+5 \Rightarrow z^{\prime}=6 x^{2}-6 x-12=0 \Rightarrow x_{1}=-1, x_{2}=2$.

It can be easily seen, that the function $z(x)$ has local maximum at $x_{1}=-1$ resp. minimum at $x_{2}=2$. The given function $f(x, y)$ has constraint local maximum at $A_{1}=[-1,-10]$ and constraint local minimum at $A_{2}=[2,-4]$.

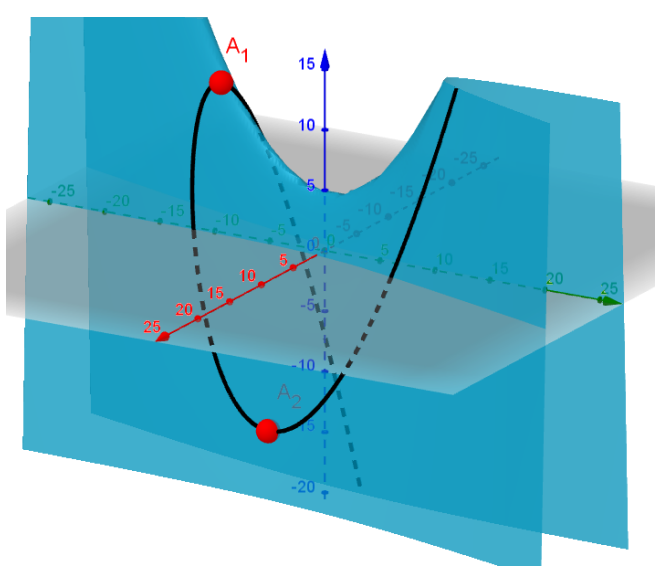

\section{Exercise 104}

Find constraint extrema of the function $z=f(x, y)$ with respect to the condition $g(x, y)=0$ :
a) $z=4 x+2 y+1, \quad y=x^{2}+x+\frac{1}{4}$
b) $z=12 x+y-3, \quad y=-x^{3}+3$

\section{Exercise 105}

Find constraint extrema of the function $z=4 x+3 y-4$ with respect to the condition $(x-1)^{2}+(y-2)^{2}=1$.

\subsection{Global extrema}

\section{Definition}

We say that a function $z=f(x, y)$ has global extremum at the point $A=\left[x_{0}, y_{0}\right]$ on closed domain $D_{f}$ if $\forall X \in D_{f}$ holds

- $f(X) \leq f(A), f$ has global maximum at the point $A$,

- $f(X) \geq f(A), f$ has global minimum at the point $A$. 


\section{Remark}

- In the case of strict inequalities we talk about strict global extremum.

- Domain $D_{f}$ is called closed, if it contains all its boundary points. Boundary point of the set $D_{f}$ is a point, such that its every neighbourhood contains points $X$ satisfying $X \in D_{f}$ and at the same time it contains points $Y$ satisfying $Y \notin D_{f}$.

\section{Algorithm}

- we find domain $D_{f}$ of the function $z=f(x, y)$,

- we find local extrema of the function $z$ on the domain $D_{f}$ from which we exclude boundary $g(x, y)=0$,

- we find constraint extrema of the function with respect to the condition $g(x, y)=0$,

- we compare all function values at extrema points, the point with the largest function value is global maximum, the point with the smallest function value is global minimum.

\section{Example 106}

Find global extrema of the function $z=f(x, y)=x^{2}-y$ defined on a square with the following vertices $[1,1],[3,1],[3,3],[1,3]$.

Domain of the function $z$ is a square, see figure below. We find local extrema of the function $z$,

$$
\frac{\partial z}{\partial x}=2 x=0, \quad \frac{\partial z}{\partial y}=-1=0 .
$$

It is clear, that the second equation has no solution, i.e. local extrema do not exist. We find equations of boundary lines representing equations of constraints,

$$
A B: y=1, x \in(1,3), B C: x=3, y \in(1,3), C D: y=3, x \in(1,3), D A: x=1, y \in(1,3) \text {. }
$$

Single equations of constraints we substitute into the function $z$ and find eventual constraint extrema,

$$
\begin{aligned}
& A B: z=x^{2}-1 \Rightarrow z^{\prime}=2 x=0 \Rightarrow x=0 \Rightarrow 0 \notin(1,3) \Rightarrow \text { extremum does not exist, } \\
& B C: z=9-y \Rightarrow z^{\prime}=-1=0 \Rightarrow \text { equation has no solution } \Rightarrow \text { extremum does not exist, } \\
& C D: z=x^{2}-3 \Rightarrow z^{\prime}=2 x=0 \Rightarrow x=0 \Rightarrow 0 \notin(1,3) \Rightarrow \text { extremum does not exist, } \\
& D A: z=1-y \Rightarrow z^{\prime}=-1=0 \Rightarrow \text { equation has no solution } \Rightarrow \text { extremum does not exist. }
\end{aligned}
$$

Finally, we have to compare function values at the vertices of the square,

$$
f(A)=0, f(B)=8, f(C)=6, f(D)=-2 \quad \Rightarrow \quad f(D)<f(A)<f(C)<f(B) .
$$

The function $z$ has global minimum with the value $z=-2$ at the point $D$, and global maximum with the value $z=8$ at the point $B$. 


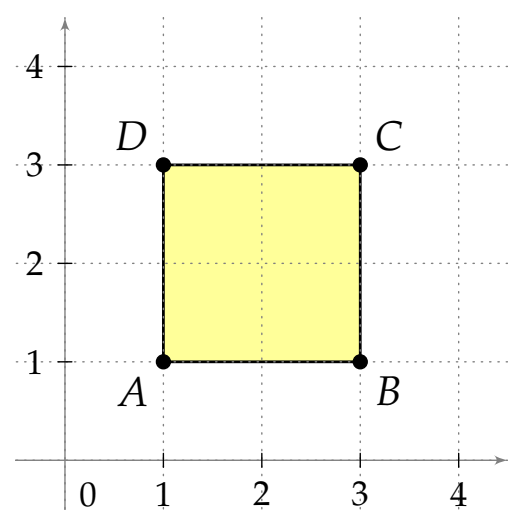

Exercise 107

Find global extrema of the function

a) $z=-x^{2}-y^{2}+2 y$ on the circle $x^{2}+y^{2} \leq 16$,

b) $z=x^{2}+y^{2}$ on the triangle with the vertices at $[0,0],[2,0]$ and $[0,1]$. 


\section{Ordinary differential equations}

\subsection{Differential equations of the $n$-th order}

Differential equations are equations in which one can find independent, dependent variables and derivatives of dependent variable with respect to the independent one. Differential equations contain derivatives. Differential equations represent a relation between unknown function and its derivatives.

\section{Definition}

Any equation of the form

$$
F\left(x, y, y^{\prime}, \ldots, y^{(n-1)}, y^{(n)}\right)=0
$$

is called $n$-th order ordinary differential equation for unknown function $y=y(x)$.

The order of the differential equation is the order of the highest derivative of unknown function $y(x)$ in the ordinary differential equation.

Solution (integral) of differential equation on the interval $I$ is every function $y(x)$, which has continuous derivatives up to order $n$ and solves the given differential equation.

We distinguish the following types of solutions:

- general solution of an $n$-th order differential equation represents the set of functions of the form

$$
\Phi\left(x, y, C_{1}, C_{2}, \ldots, C_{n}\right)=0, \quad \text { resp. } y=\varphi\left(x, C_{1}, C_{2}, \ldots, C_{n}\right),
$$

i.e. the set of functions containing $n$ real constants $C_{1}, C_{2}, \ldots, C_{n}$,

- particular solution $y_{p}$ is one concrete solution, which can be obtained from a general solution by the choice or calculation of integration constants $C_{1}, C_{2}, \ldots, C_{n}$,

- singular solution is a solution, which cannot be obtained from a general solution by any choice of integration constants $C_{1}, C_{2}, \ldots, C_{n}$.

Remark

- For simplicity, instead of ordinary differential equations we use words differential equations. There exist also partial differential equations, but those equations will not be discussed. Thus, there is no need to distinguish between ordinary and particular differential equations.

- It is common to use instead of ordinary differential equations the abbreviation ODE. 
Example 108

Find at least one particular solution of ODE $y^{\prime \prime \prime}=6 x-6$.

Given differential equation can be integrated directly, i.e. one can integrate simultaneously both sides of the equation. Recall a relation between derivatives and differentials, $y^{\prime}=$ $\frac{\mathrm{d}}{\mathrm{d} x}(y)$.

$$
y^{\prime \prime}=\int y^{\prime \prime \prime} \mathrm{d} x=\int(6 x-6) \mathrm{d} x \Rightarrow y^{\prime \prime}=3 x^{2}-6 x+C_{1} .
$$

Using the same way, we can subsequently reduce the order of the given ODE. Thus,

$$
\begin{gathered}
y^{\prime}=\int y^{\prime \prime} \mathrm{d} x=\int\left(3 x^{2}-6 x+C_{1}\right) \mathrm{d} x \quad \Rightarrow \quad y^{\prime}=x^{3}-3 x^{2}+C_{1} x+C_{2}, \\
y=\int y^{\prime} \mathrm{d} x=\int\left(x^{3}-3 x^{2}+C_{1} x+C_{2}\right) \mathrm{d} x \Rightarrow y=\frac{x^{4}}{4}-x^{3}+C_{1} \frac{x^{2}}{2}+C_{2} x+C_{3} .
\end{gathered}
$$

We see three integration constants $C_{1}, C_{2}, C_{3}$ in the general solution.

By the particular solution we mean a concrete curve. This curve can be obtained by arbitrary choice of the integration constants $C_{1}, C_{2}$ and $C_{3}$, e.g. for $C_{1}=2, C_{2}=3$ a $C_{3}=5$ we get the particular solution of the form

$$
y_{p}=\frac{x^{4}}{4}-x^{3}+x^{2}+3 x+5
$$

Note that in concrete practical situations the choice of integration constants is not arbitrary and is strictly connected with the solved problem.

\subsection{First order ordinary differential equations}

Definition

Differential equation of the type

$$
F\left(x, y, y^{\prime}\right)=0 \quad \text { or } \quad y^{\prime}=f(x, y)
$$

is called first order ODE.

\section{Remark}

A solution of the first order $\operatorname{ODE} F\left(x, y, y^{\prime}\right)=0$ is also called integral of ODE and its graph in the coordinate plane $x y$ is called integral curve. It is common and very frequent that integral curves and therefore general solutions may be represented in the implicit form.

\section{Geometrical interpretation of the first order ODE $y^{\prime}=f(x, y)$}

Let us consider $x, y$ as coordinates of the point $[x, y]$ in the $x y$-plane. To every point $[x, y]$ one can assign the value $f(x, y)$, which is related to the derivative $y^{\prime}$. Geometrically, derivative represents a slope (direction), thus one can define slope field $\{(x, y, f(x, y))\}$. 
Ordered triples $(x, y, f(x, y))$ are called linear elements and we illustrate them by short segments with the center at the point $(x, y)$ and the slope $f(x, y)$.

Integral curves of the equation $y^{\prime}=f(x, y)$ have a tangent line at every point oriented in accordance with the slope field. Curves in which derivative is constant $\left(y^{\prime}=k, k \in \mathbb{R}\right)$, are called isoclines. Slope field is a system of linear segments tangent to integral curves.

To clearly predict the future state, it is necessary to know the current state of the system. In practical tasks, we are often not interested in all solutions of a given task, but we only seek for such solutions which satisfy certain conditions. One of the possible condition is initial condition. If an initial condition is set for a given differential equation, we talk about Cauchy problem. It is the basic task in the theory of ordinary differential equations.

\section{Definition}

As Cauchy problem (initial value problem) for $\operatorname{ODE} F\left(x, y, y^{\prime}\right)=0$ we shall mean the following task

$$
\left\{\begin{aligned}
y^{\prime} & =f(x, y) \\
y\left(x_{0}\right) & =y_{0} .
\end{aligned}\right.
$$

The solution of Cauchy problem is such a solution $y=y(x)$ of ODE, which is defined on an interval $I$ and satisfies the initial condition $y\left(x_{0}\right)=y_{0}$ (where $x_{0} \in I$ ).

\subsection{Slope field}

Example 109

Use isoclines to draw a slope filed of the first order ODE $y^{\prime}=x-\sqrt{y}$.

The function $f(x, y)=x-\sqrt{y}$ is defined $\forall x \in \mathbb{R}$ and $y \geq 0$.

We find isoclines in such a way that we set the function equal to a constant and evaluate $y$.

$$
x-\sqrt{y}=k \Rightarrow \sqrt{y}=x-k \Rightarrow y=(x-k)^{2} ; k \in \mathbb{R}, x \geq k .
$$

It is a right part of a parabola curve with vertex at the point $[k, 0]$.
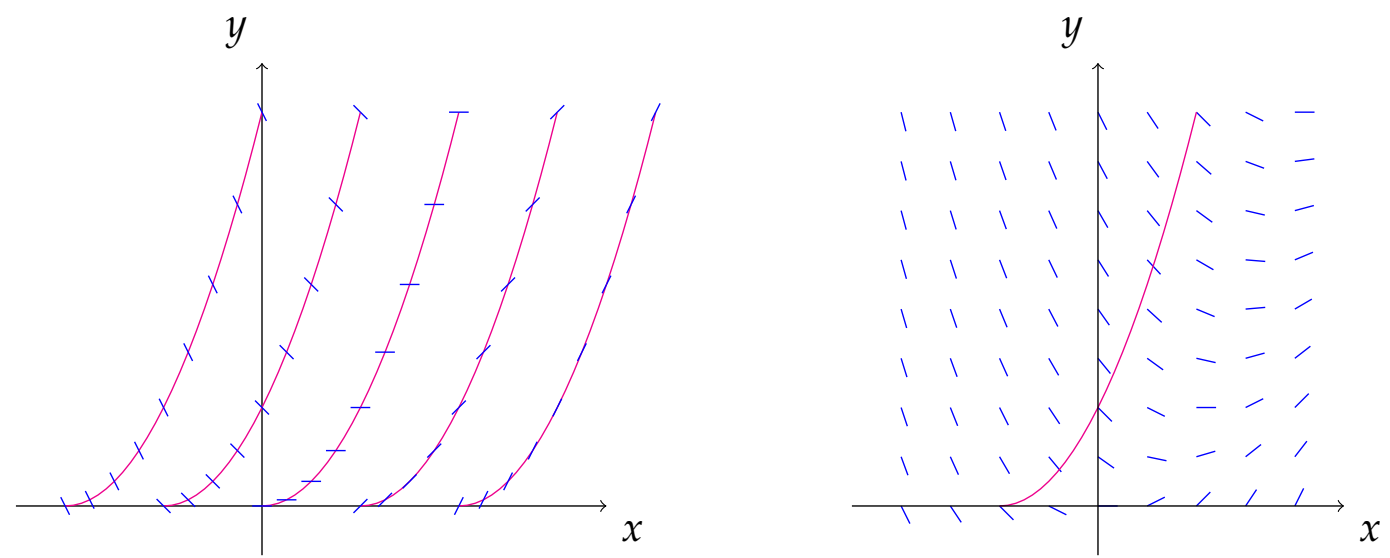

Isoclines of the equation $y^{\prime}=x-\sqrt{y}$. Slope field of the equation $\quad y^{\prime}=x-\sqrt{y}$. 


\subsection{Cauchy problem}

Example 110

Solve Cauchy problem $y^{\prime}(y-x)=(y-x) \sin x, y(0)=2$.

The given ODE can be simplified $y^{\prime}=f(x, y)$, the equation one can divide by $(y-x)$ if $y \neq$ $x$. We get the equation in the form $y^{\prime}=f(x)$, i.e. we seek the appropriate antiderivative

$$
y^{\prime}=\sin x \Rightarrow y=\int \sin x d x \quad \Rightarrow \quad y=-\cos x+C, \quad C \in R
$$

We found general solution. The initial condition $y(0)=2$ we substitute into the general solution and evaluate the integration constant $C$.

$$
\begin{gathered}
x_{0}=0, \quad y_{0}=2 \Rightarrow 2=-\cos 0+C \Rightarrow 2=-1+C \Rightarrow C=3 \\
y_{p}=-\cos x+3 \text { is our particular solution. }
\end{gathered}
$$

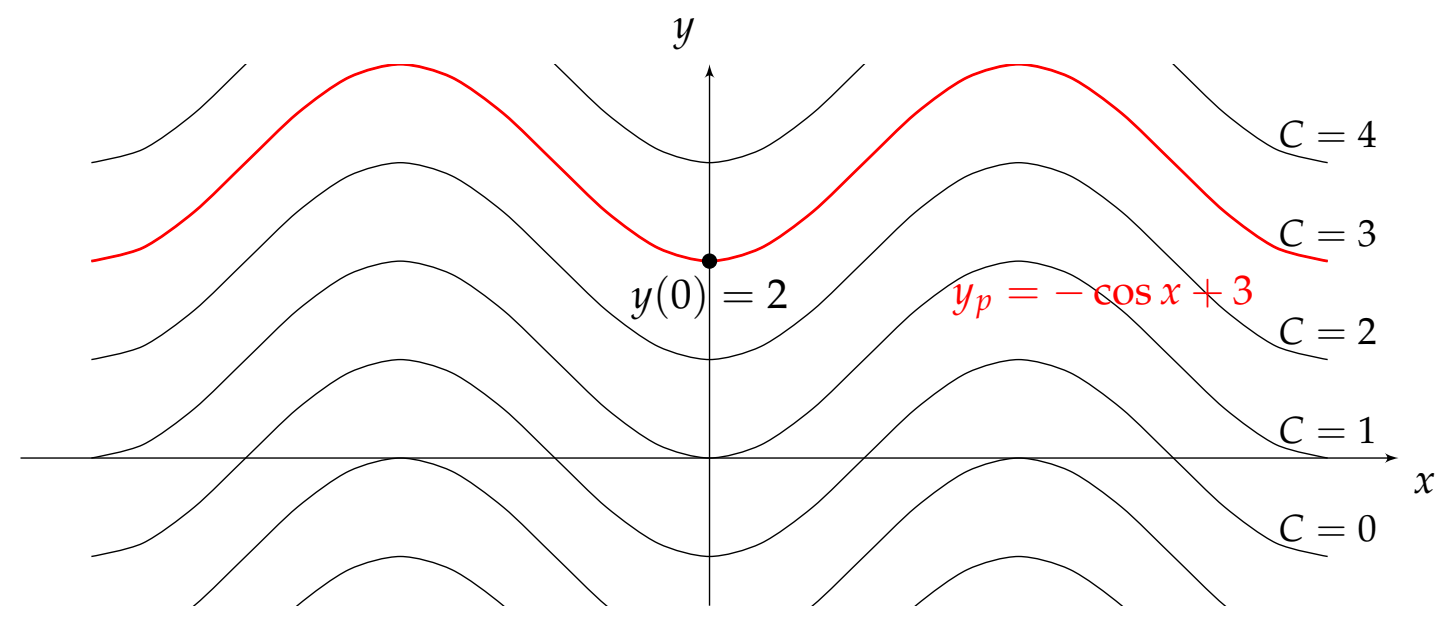

Particular solution $y_{p}$

Note that the function $y=x$ is also a solution of the given ODE, but it can not be obtained by any choice of the integration constant. It is a singular solution. This solution does not satisfy the initial condition.

\subsection{Separable differential equations}

\section{Definition}

By differential equation with separated variables we shall mean every equation which can be expressed in the form

$$
Q(y) y^{\prime}=P(x), \quad \text { i.e. } \quad Q(y) \mathrm{d} y=P(x) \mathrm{d} x,
$$

if we use instead of derivative $y^{\prime}$ quotient $\frac{\mathrm{d} y}{\mathrm{~d} x}$. 
It can be immediately seen that the variables are separated on both sides of the equation and one can directly integrate them. This leads to

$$
\int Q(y) \mathrm{d} y=\int P(x) \mathrm{d} x+C
$$

Note that we consider the only one integration constant, usually we add this integration constant to the right side of the equation, where the independent variable $x$ is presented.

In practice, we encounter a lot of problems that can be converted to a separated differential equation with the help of simple manipulations. Such equations are called separable. We distinguish three types of separable ODE:

- $y^{\prime}=f(x) g(y)$,

- $y^{\prime}=f(a x+b y+c)$,

- $y^{\prime}=f\left(\frac{y}{x}\right)$, called homogeneous ODE.

\subsection{Separable ODE of the type $y^{\prime}=f(x) g(y)$}

The equation $y^{\prime}=f(x) g(y)$ assuming $g(y) \neq 0$ and using identity $y^{\prime}=\frac{\mathrm{d} y}{\mathrm{~d} x}$ can be rewritten as follows:

$$
\frac{\mathrm{d} y}{g(y)}=f(x) \mathrm{d} x
$$

which is ODE with separated variables. Its general solution can be written as

$$
\int \frac{\mathrm{d} y}{g(y)}=\int f(x) \mathrm{d} x+C
$$

\section{Remark}

The shape of the general solution should be adjusted in some case. We get a logarithmic function on the left-hand side after the integration. In this case, as a logarithmic function by the relation $A=\ln \mathrm{e}^{A}$ we also express the right-hand side of the solution.

Assume that the general solution is of the form $\ln m(y)=n(x)+C$. We introduce a logarithmic function on the right-hand side

$$
\ln m(y)=\ln \mathrm{e}^{n(x)+C} \Rightarrow \ln m(y)=\ln \left(\mathrm{e}^{n(x)} \mathrm{e}^{C}\right) .
$$

From the equality of logarithms it follows

$$
m(y)=\mathrm{e}^{n(x)} \mathrm{e}^{C}
$$

Without loss of generality it is common to label new constant $\mathrm{e}^{C}$ like the original constant " $\mathrm{e}^{\mathrm{C}}=\mathrm{C}^{\prime}$. Thus the solution takes the form

$$
m(y)=C \mathrm{e}^{n(x)}
$$




\section{Example 111}

Find the general solution of ODE $y^{\prime}=\frac{x-\mathrm{e}^{-x}}{y+\mathrm{e}^{y}}$.

We rewrite the ODE to the form $y^{\prime}=\left(x-\mathrm{e}^{-x}\right) \cdot \frac{1}{y+\mathrm{e}^{y}}$ where it is easy to see that the equation is of form $y^{\prime}=f(x) g(y)$. We are going to solve it by the method of separation of variables,

$$
y^{\prime}=\frac{x-\mathrm{e}^{-x}}{y+\mathrm{e}^{y}} \Rightarrow \frac{\mathrm{d} y}{\mathrm{~d} x}=\frac{x-\mathrm{e}^{-x}}{y+\mathrm{e}^{y}} \Rightarrow\left(y+\mathrm{e}^{y}\right) \mathrm{d} y=\left(x-\mathrm{e}^{-x}\right) \mathrm{d} x .
$$

After the integration of both sides of the equation we get

$$
\int\left(y+\mathrm{e}^{y}\right) \mathrm{d} y=\int\left(x-\mathrm{e}^{-x}\right) \mathrm{d} x+C \quad \Rightarrow \quad \frac{y^{2}}{2}+\mathrm{e}^{y}=\frac{x^{2}}{2}+\mathrm{e}^{-x}+C .
$$

The general solution takes the form

$$
\frac{y^{2}}{2}-\frac{x^{2}}{2}+\mathrm{e}^{y}-\mathrm{e}^{-x}=C .
$$

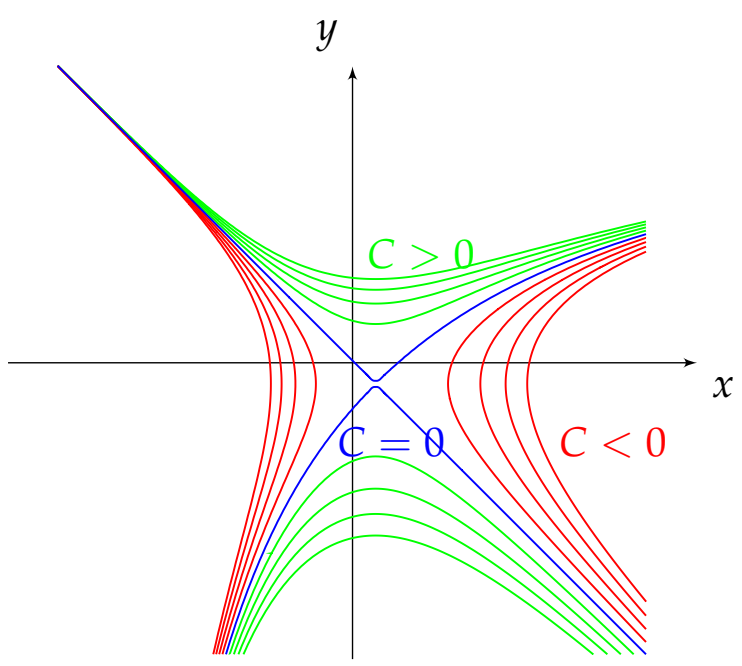

General solution $y$

Example 112

Solve the Cauchy problem $\frac{y^{\prime}}{y}=-2 \sin x, y(\pi)=1$.

It is a separable ODE,

$$
\frac{y^{\prime}}{y}=-2 \sin x \quad \Rightarrow \quad \frac{1}{y} \frac{\mathrm{d} y}{\mathrm{~d} x}=-2 \sin x \quad \Rightarrow \quad \frac{1}{y} \mathrm{~d} y=-2 \sin x \mathrm{~d} x .
$$

After the integration we get

$$
\int \frac{1}{y} \mathrm{~d} y=-2 \int \sin x \mathrm{~d} x+C \Rightarrow \ln |y|=2 \cos x+C
$$


We rewrite the general solution

$$
\ln |y|=2 \cos x+C \Rightarrow y=C \mathrm{e}^{2 \cos x} .
$$

We find the value for $C$ using the initial condition, i.e. $x=\pi, y=1$,

$$
1=C \mathrm{e}^{2 \cos \pi} \Rightarrow C=\mathrm{e}^{2} \text {. }
$$

Finally, the particular solution takes the form

$$
y_{p}=\mathrm{e}^{2} \mathrm{e}^{2 \cos x} \Rightarrow y_{p}=\mathrm{e}^{2 \cos x+2} .
$$

\section{Example 113}

How long does it take for the temperature of the heated body to drop from $90{ }^{\circ} \mathrm{C}$ to $50{ }^{\circ} \mathrm{C}$, if the temperature of environment is equal to $25^{\circ} \mathrm{C}$ and in the first 10 minutes the body cooled down to $73^{\circ} \mathrm{C}$.

Speed of cooling of a body is represented by a decrease of temperature $\tau$ in time $t$ and is given by the derivative $\frac{\mathrm{d} \tau}{\mathrm{d} t}$. Newton's law of cooling states that speed of cooling is proportional to a difference between the temperature of the body and the temperature of environment. If we assume that the temperature of environment is constant, the differential equation of cooling takes the form

$$
\frac{\mathrm{d} \tau}{\mathrm{d} t}=-k\left(\tau-\tau_{0}\right)
$$

where $\tau$ is temperature of the body, $\tau_{0}$ is temperature of environment and $k>0$ is coefficient of proportionality.

The equation can be solved by separation of variables method:

$$
\frac{\mathrm{d} \tau}{\tau-\tau_{0}}=-k \mathrm{~d} t \quad \Rightarrow \quad \ln \left|\tau-\tau_{0}\right|=-k t+c \Rightarrow \tau=\tau_{0}+c_{1} \mathrm{e}^{-k t}
$$

We use the initial condition to find the value for the integration constant $c_{1}$, at $t_{0}=0$ is $\tau=90$ and $\tau_{0}=25$,

$$
90=25+c_{1} \mathrm{e}^{-k \cdot 0} \Rightarrow c_{1}=65 .
$$

We see that at the time $t=10$ is $\tau=73$ and $\tau_{0}=25$, we use this values to find $k$ and finally the relation between $\tau$ and $t$ :

$$
\begin{aligned}
73=25+65 \mathrm{e}^{-k \cdot 10} & \Rightarrow \mathrm{e}^{-k \cdot 10}=\frac{48}{65} \Rightarrow\left(\mathrm{e}^{-k}\right)^{10}=\frac{48}{65} \Rightarrow \mathrm{e}^{-k}=\left(\frac{48}{65}\right)^{\frac{1}{10}} \\
& \Rightarrow \tau=25+65 \cdot\left(\frac{48}{65}\right)^{\frac{t}{10}} .
\end{aligned}
$$

We find the time $t$ of cooling the body for $\tau=50$ :

$$
\begin{aligned}
50=25+65 \cdot\left(\frac{48}{65}\right)^{\frac{t}{10}} & \Rightarrow \frac{5}{13}=\left(\frac{48}{65}\right)^{\frac{t}{10}} \Rightarrow\left(\frac{5}{13}\right)^{10}=\left(\frac{48}{65}\right)^{t} \\
& \Rightarrow t=\frac{10 \ln \frac{5}{13}}{\ln \frac{48}{65}}=31.5 \mathrm{~min} .
\end{aligned}
$$

The body is cooled down to $50{ }^{\circ} \mathrm{C}$ in 31 minutes and 30 seconds. 


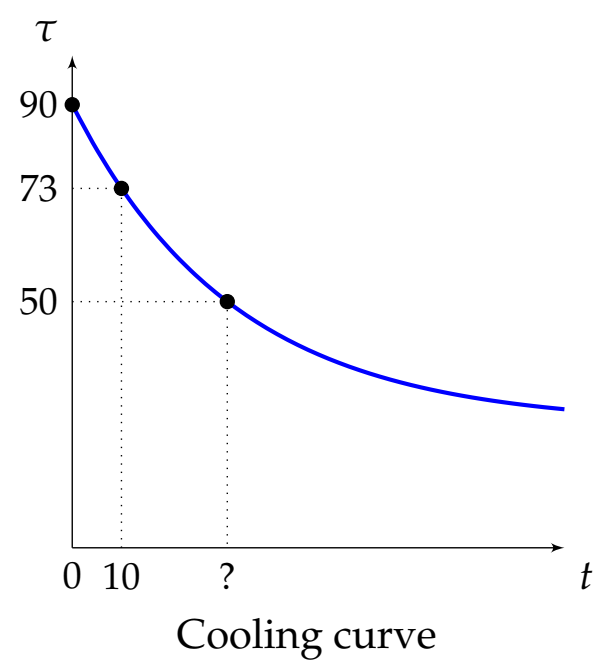

Exercise 114

Solve the differential equations:
a) $y^{\prime} \sin x=y \cos x$
b) $x^{2} y^{\prime}-y^{2}=1$
c) $2 x y y^{\prime}=x+2$

\section{Exercise 115}

Solve the Cauchy problems:
a) $y^{\prime} \sin x=y \ln y, y\left(\frac{\pi}{2}\right)=\mathrm{e}$
b) $y-x y^{\prime}=5\left(1+x^{2} y^{\prime}\right), y(1)=1$

\subsection{Separable ODE of the type $y^{\prime}=f(a x+b y+c)$}

Differential equations of the form $y^{\prime}=f(a x+b y+c)$, where $b \neq 0$, can be transformed to a differential equation with separated variables by means of the substitution $u(x)=$ $a x+b y+c$. Substitution gives the formula for the derivative $y^{\prime}$.

We differentiate the equality $u(x)=a x+b y+c$ with respect to $x$ and express $y^{\prime}$,

$$
u^{\prime}=a+b y^{\prime} \Rightarrow y^{\prime}=\frac{u^{\prime}-a}{b} .
$$

After the substitution into the original differential equation we get a differential equation with separable variables of the form $u^{\prime}=f(x) g(u)$,

$$
\frac{u^{\prime}-a}{b}=f(u) \quad \Rightarrow \quad u^{\prime}=a+b f(u) .
$$

For $a+b f(u) \neq 0$ we get the equation

$$
\frac{1}{a+b f(u)} u^{\prime}=1 .
$$

Its general solution then takes the form

$$
\int \frac{\mathrm{d} u}{a+b f(u)}=x+C .
$$




\section{Example 116}

Find the general solution of ODE $y^{\prime}=3 x-2 y+5$.

It is a separable ODE of the type $y^{\prime}=f(a x+b y+c)$, where $a=3, b=-2, c=5$. We use the substitution,

$$
u=3 x-2 y+5 \Rightarrow u^{\prime}=3-2 y^{\prime} \Rightarrow y^{\prime}=\frac{3-u^{\prime}}{2} .
$$

After the substitution, the original ODE becomes:

$$
\frac{3-u^{\prime}}{2}=u
$$

This is a separable ODE of the type $u^{\prime}=f(x) g(u)$,

$$
u^{\prime}=3-2 u \Rightarrow \frac{\mathrm{d} u}{\mathrm{~d} x}=3-2 u \Rightarrow \frac{\mathrm{d} u}{3-2 u}=\mathrm{d} x
$$

After the integration we get

$$
\int \frac{\mathrm{d} u}{3-2 u}=\int \mathrm{d} x+C \Rightarrow \quad-\frac{1}{2} \ln |3-2 u|=x+C \quad \Rightarrow \quad \ln |3-2 u|=-2 x+C .
$$

The general solution for unknown function $u$ can be written as follows,

$$
3-2 u=C \mathrm{e}^{-2 x} \Rightarrow u=C \mathrm{e}^{-2 x}+\frac{3}{2} .
$$

Next, we return to the original variable $y$ :

$$
3 x-2 y+5=C \mathrm{e}^{-2 x}+\frac{3}{2} .
$$

The general solution for the unknown function $y$ then takes the form $y=C \mathrm{e}^{-2 x}+\frac{3}{2} x+\frac{7}{4}$.

\section{Example 117}

Find the general solution of ODE $y^{\prime}=\cos (x-y)$.

It is an equation of the type $y^{\prime}=f(a x+b y+c)$, where $a=1, b=-1, c=0$. The substitution takes the form,

$$
u=x-y \quad \Rightarrow \quad u^{\prime}=1-y^{\prime} \quad \Rightarrow \quad y^{\prime}=1-u^{\prime} .
$$

The original ODE becomes

$$
1-u^{\prime}=\cos u \quad \Rightarrow \quad u^{\prime}=1-\cos u \text {. }
$$

We replace $u^{\prime}$ by $\frac{\mathrm{d} u}{\mathrm{~d} x}$ and write the ODE in the separated form

$$
\frac{\mathrm{d} u}{\mathrm{~d} x}=1-\cos u \Rightarrow \int \frac{\mathrm{d} u}{1-\cos u}=\int \mathrm{d} x+C
$$


We calculate the integral on the left-hand side separately by the universal trigonometric substitution:

$$
\int \frac{\mathrm{d} u}{1-\cos u}=\left|\begin{array}{c}
\tan \frac{u}{2}=t \\
\cos u=\frac{1-t^{2}}{1+t^{2}} \\
\mathrm{~d} u=\frac{2}{1+t^{2}} \mathrm{~d} t
\end{array}\right|=\int \frac{1}{1-\frac{1-t^{2}}{1+t^{2}}} \frac{2}{1+t^{2}} \mathrm{~d} t=\int \frac{\mathrm{d} t}{t^{2}}=-\frac{1}{t}=-\frac{1}{\tan \frac{u}{2}}=-\cot \frac{u}{2} .
$$

The general solution for the unknown function $u$ is:

$$
-\cot \frac{u}{2}=x+C \text {. }
$$

We return to the original variable and get general solution in the form

$$
-x-\cot \frac{x-y}{2}=C \quad \Rightarrow \quad x+\cot \frac{x-y}{2}=C \text {. }
$$

\section{Exercise 118}

Solve the differential equations
a) $(x+y) y^{\prime}=1$
c) $y^{\prime}=\sin ^{2}(x-y)$
b) $y^{\prime}-3 y=4 x+1$
d) $y^{\prime} \sqrt{1+x+y}=x+y+1$

\subsection{Separable ODE of the type $y^{\prime}=f\left(\frac{y}{x}\right)$}

\section{Definition}

The differential equation $F\left(x, y, y^{\prime}\right)=0$ is called homogeneous, if it can be written in the form

$$
y^{\prime}=f\left(\frac{y}{x}\right)
$$

for $x \neq 0$.

Homogeneous differential equations can be formed to the separated differential equations by means of the substitution

$$
y=z x,
$$

where $z=z(x)$. From the substitution $y=z x$, i.e $z=\frac{y}{x}$, we compute the formula for substitution of the derivative $y^{\prime}$ :

$$
y^{\prime}=z^{\prime} x+z
$$

After substitution the original equation becomes separable differential equation for the function $z=z(x)$, if moreover $f(z)-z \neq 0$.

$$
\begin{aligned}
z^{\prime} x+z & =f(z) \\
z^{\prime} x & =f(z)-z \\
z^{\prime} & =\frac{1}{x}(f(z)-z) \\
\frac{1}{f(z)-z} \mathrm{~d} z & =\frac{1}{x} \mathrm{~d} x
\end{aligned}
$$


Then general solution takes the form

$$
\int \frac{\mathrm{d} z}{f(z)-z}=\ln |x|+C
$$

\section{Remark}

Recall the fact when the function $f(x, y)$ on a region $\Omega \in \mathbb{R}^{2}$ is called homogeneous of degree $k$ and we show how this notion is connected with homogeneous differential equations.

A function $f(x, y)$ is called homogeneous function of degree $k(k \in \mathbb{N})$ on $\Omega$ if at any point $[x, y] \in \Omega$ and for arbitrary $t \neq 0$ it holds

$$
f(t x, t y)=t^{k} f(x, y) .
$$

If we assume that functions $P(x, y), Q(x, y)$ are homogeneous of the same degree $k$, then the equation $P(x, y)+Q(x, y) y^{\prime}=0$ is homogeneous differential equation.

Thus

$$
\begin{gathered}
P(t x, t y)=t^{k} P(x, y) \quad \wedge \quad Q(t x, t y)=t^{k} Q(x, y) \\
\frac{P(t x, t y)}{Q(t x, t y)}=\frac{t^{k} P(x, y)}{t^{k} Q(x, y)} \Rightarrow \frac{P(t x, t y)}{Q(t x, t y)}=\frac{P(x, y)}{Q(x, y)}
\end{gathered}
$$

The equation $P(x, y)+Q(x, y) y^{\prime}=0$ can be rewritten for $Q(x, y) \neq 0$ onto the form

$$
y^{\prime}=-\frac{P(x, y)}{Q(x, y)} \Rightarrow y^{\prime}=-\frac{P(t x, t y)}{Q(t x, t y)}
$$

and from this equation for $t=\frac{1}{x}, x \neq 0$ we get

$$
y^{\prime}=-\frac{P\left(1, \frac{y}{x}\right)}{Q\left(1, \frac{y}{x}\right)} \quad \Rightarrow \quad y^{\prime}=f\left(\frac{y}{x}\right),
$$

which is homogeneous differential equation.

\section{Example 119}

Find the general solution of the ODE $x y^{\prime}-y=2 \sqrt{x y}$.

$$
x y^{\prime}=2 \sqrt{x y}+y \quad \Rightarrow \quad y^{\prime}=\frac{2 \sqrt{x y}+y}{x}=2 \sqrt{\frac{y}{x}}+\frac{y}{x}
$$

It is homogeneous ODE $y^{\prime}=f\left(\frac{y}{x}\right)$.

Substitution:

$$
\begin{aligned}
& \frac{y}{x}=z \\
& y^{\prime}=z^{\prime} x+z,
\end{aligned}
$$


where $z=z(x)$.

We substitute into the equation $y^{\prime}=2 \sqrt{\frac{y}{x}}+\frac{y}{x}$ and separate the new variables,

$$
z^{\prime} x+z=2 \sqrt{z}+z \quad \Rightarrow \quad z^{\prime} x=2 \sqrt{z} \quad \Rightarrow \quad \frac{\mathrm{d} z}{\mathrm{~d} x}=\frac{2}{x} \cdot \sqrt{z} \quad \Rightarrow \quad \frac{1}{\sqrt{z}} \mathrm{~d} z=\frac{2}{x} \mathrm{~d} x
$$

Now, we integrate both sides of the equation

$$
\begin{aligned}
\int \frac{1}{\sqrt{z}} \mathrm{~d} z=\int \frac{2}{x} \mathrm{~d} x+C & \Rightarrow \int z^{-\frac{1}{2}} \mathrm{~d} z=\int \frac{2}{x} \mathrm{~d} x+C \Rightarrow \frac{z^{\frac{1}{2}}}{\frac{1}{2}}=2 \ln |x|+C \\
& \Rightarrow \sqrt{z}=\ln |x|+C
\end{aligned}
$$

We rewrite found solution for unknown function $z(x)$

$$
z=(\ln |x|+C)^{2}
$$

and return to the original unknown $y(x)$

$$
\frac{y}{x}=(\ln |x|+C)^{2} \Rightarrow y=x(\ln |x|+C)^{2} .
$$

Example 120

Find the general solution of the ODE $y^{\prime}=\frac{3 y-2 x}{x+y}$.

We check homogeneity of the given ODE.

$$
y^{\prime}=\frac{3 y-2 x}{x+y} \Rightarrow y^{\prime}=\frac{x\left(3 \frac{y}{x}-2\right)}{x\left(1+\frac{y}{x}\right)} \Rightarrow y^{\prime}=\frac{3 \frac{y}{x}-2}{1+\frac{y}{x}}
$$

Substitution:

$$
\frac{y}{x}=z \quad \Rightarrow \quad y^{\prime}=z^{\prime} x+z, \quad \text { where } z=z(x) .
$$

We substitute into the equation $y^{\prime}=\frac{3 \frac{y}{x}-2}{1+\frac{y}{x}}$.

$$
\begin{aligned}
z^{\prime} x+z=\frac{3 z-2}{1+z} & \Rightarrow z^{\prime} x=\frac{3 z-2}{1+z}-z \Rightarrow \frac{\mathrm{d} z}{\mathrm{~d} x}=-\frac{1}{x} \cdot \frac{z^{2}-2 z+2}{1+z} \\
& \Rightarrow \frac{1+z}{z^{2}-2 z+2} \mathrm{~d} z=-\frac{1}{x} \mathrm{~d} x
\end{aligned}
$$

Now, we integrate both sides of the equation. Stress that on the left-hand side of the equation, we integrate partial fraction of the second type:

$$
\begin{aligned}
\int \frac{1+z}{z^{2}-2 z+2} \mathrm{~d} z & =-\int \frac{\mathrm{d} x}{x}+C \\
2 \int \frac{1}{(z-1)^{2}+1} \mathrm{~d} z+\frac{1}{2} \int \frac{(2 z-2)}{z^{2}-2 z+2} \mathrm{~d} z & =-\ln |x|+C \\
2 \arctan (z-1)+\frac{1}{2} \ln \left|z^{2}-2 z+2\right| & =-\ln |x|+C
\end{aligned}
$$


We return substitution $z=\frac{y}{x}$ and get general solution for the function $y(x)$ :

$$
4 \arctan \left(\frac{y}{x}-1\right)+\ln \left|\left(\frac{y}{x}\right)^{2}-2 \frac{y}{x}+2\right|=-2 \ln |x|+C .
$$

Exercise 121

Solve the differential equations
a) $x^{2} y^{\prime}=y^{2}+x y$
b) $x y^{\prime}=y(\ln y-\ln x)$

\subsection{Linear differential equations of the first order}

By linear differential equations we shall mean equations which are linear with respect to an unknown function and its derivatives. Linear differential equations are very important because a lot of practical problems can be modelled by such equations.

\section{Definition}

We call linear ordinary differential equation (shortly LODE) of the first order every equation of the form

$$
y^{\prime}+p(x) y=q(x)
$$

where $p(x), q(x)$ are continuous functions on some interval $[a, b]$. Moreover,

- if $q(x)=0$, we talk about homogeneous (shortened) LODE,

- if $q(x) \neq 0$, we talk about non-homogeneous (complete) LODE.

Remark

Examples of linear equations:

- $x y^{\prime}-y=x$

- $y^{\prime}-\ln x^{2} \cdot y=\sin x$

- $2 x y^{\prime}-\frac{y}{x}-2=0$
Examples of non-linear equations:

- $x y^{\prime} y=x$

- $y^{\prime}-x y^{2}=\sin x$

- $2 x y^{\prime}-\frac{x}{y}-2 \sin y=0$

Homogeneous LODE $y^{\prime}+p(x) \cdot y=0$ is also separable ODE $y^{\prime}=-p(x) y$, which we know how to solve. To every complete LODE $y^{\prime}+p(x) y=q(x)$ there exists associated shortened equation $y^{\prime}+p(x) y=0$.

\section{Theorem}

Homogeneous LODE $y^{\prime}+p(x) y=0$ has on the interval $[a, b]$ general solution of the form

$$
\hat{y}=C \mathrm{e}^{-\int p(x) \mathrm{d} x}, \quad C \in \mathbb{R} .
$$




\section{Theorem}

General solution of complete LODE has the form

$$
y(x)=\hat{y}(x)+v(x),
$$

where $\hat{y}(x)$ is general solution of associated homogeneous LODE and $v(x)$ is a particular solution of the complete LODE.

The function $v(x)$ is called particular integral of complete LODE.

To find a solution of non-homogeneous LODE (LODE with non-trivial right-hand side) one can use a method called variation of a constant, the integration constant $C$ is replaced by some unknown function depending on $x, C=C(x)$.

Algorithm:

1. We find the solution of associated homogeneous LODE using method of separation of variables

$$
\begin{aligned}
y^{\prime}+p(x) y & =0 \\
\hat{y} & =C \mathrm{e}^{-\int p(x) \mathrm{d} x}, C \in \mathbb{R} .
\end{aligned}
$$

2. General solution of non-homogeneous LODE has the same form as the solution of associated homogeneous LODE. There is a huge difference, the integration constant $C$ is not a constant any more, but it must be treated as a function $C(x)$ such that the function $y=C(x) \mathrm{e}^{-\int p(x) \mathrm{d} x}$ is a solution of the original non-homogeneous LODE.

How to find the function $C(x)$ :

We calculate a derivative of the estimate solution

$$
\begin{aligned}
y & =C(x) \mathrm{e}^{-\int p(x) \mathrm{d} x} \\
y^{\prime} & =C^{\prime}(x) \mathrm{e}^{-\int p(x) \mathrm{d} x}-C(x) \mathrm{e}^{-\int p(x) \mathrm{d} x} p(x) .
\end{aligned}
$$

We substitute the function $y$ and its derivative $y^{\prime}$ into the original non-homogeneous LODE and we get the equation for the unknown function $C^{\prime}(x)$.

$$
\underbrace{C^{\prime}(x) \mathrm{e}^{-\int p(x) \mathrm{d} x}-C(x) \mathrm{e}^{-\int p(x) \mathrm{d} x} p(x)}_{y^{\prime}}+p(x) \cdot \underbrace{C(x) \mathrm{e}^{-\int p(x) \mathrm{d} x}}_{y}=q(x) .
$$

The terms with $C(x)$ must vanish. This is a control point. If not, we need to check our calculation because there is a mistake somewhere. After that we differentiate equation for the unknown function $C(x)$.

$$
C^{\prime}(x) \mathrm{e}^{-\int p(x) \mathrm{d} x}=q(x) \quad \Rightarrow \quad C^{\prime}(x)=q(x) \mathrm{e}^{\int p(x) \mathrm{d} x}
$$

We can integrate both sides and get the function $C(x)$ with new integration constant, it is possible to use the same letter $C$ :

$$
C(x)=\int q(x) \mathrm{e}^{\int p(x) \mathrm{d} x} \mathrm{~d} x+C,
$$


which we substitute into the estimated solution $y=C(x) \mathrm{e}^{-\int p(x) \mathrm{d} x}$ and get the general solution of the original equation

$$
\begin{aligned}
y & =\left(\int q(x) \mathrm{e}^{\int p(x) \mathrm{d} x} \mathrm{~d} x+C\right) \mathrm{e}^{-\int p(x) \mathrm{d} x} \\
& =\underbrace{C \mathrm{e}^{-\int p(x) \mathrm{d} x}}_{\hat{y}(x)}+\underbrace{\mathrm{e}^{-\int p(x) \mathrm{d} x} \int q(x) \mathrm{e}^{\int p(x) \mathrm{d} x} \mathrm{~d} x}_{v(x)} .
\end{aligned}
$$

Example 122

Find the general solution of LODE $y^{\prime}-y=\mathrm{e}^{2 x}$.

Associated homogeneous LODE has the form $y^{\prime}-y=0$. It is a separable ODE, thus

$$
\frac{\mathrm{d} y}{\mathrm{~d} x}=y \quad \Rightarrow \quad \int \frac{\mathrm{d} y}{y}=\int \mathrm{d} x+C \Rightarrow \ln |y|=x+C \quad \Rightarrow \quad \hat{y}=C \mathrm{e}^{x}
$$

We proceed with the variation of the integration constant. Assume that $C=C(x)$, then

$$
y=C(x) \mathrm{e}^{x}
$$

and its derivative is

$$
y^{\prime}=C^{\prime}(x) \mathrm{e}^{x}+C(x) \mathrm{e}^{x}
$$

After the substitution into the original ODE we get

$$
\begin{gathered}
C^{\prime}(x) \mathrm{e}^{x}+C(x) \mathrm{e}^{x}-C(x) \mathrm{e}^{x}=\mathrm{e}^{2 x}, \\
C^{\prime}(x) \mathrm{e}^{x}=\mathrm{e}^{2 x} \quad \Rightarrow \quad C^{\prime}(x)=\mathrm{e}^{x}
\end{gathered}
$$

We can directly integrate,

$$
C(x)=\int \mathrm{e}^{x} \mathrm{~d} x=\mathrm{e}^{x}+C .
$$

After the substitution of $y=C(x) \mathrm{e}^{x}$ into the general solution of homogeneous equation we get the general solution of the original LODE

$$
y=\left(\mathrm{e}^{x}+C\right) \mathrm{e}^{x}=C \mathrm{e}^{x}+\mathrm{e}^{2 x}=\hat{y}+v .
$$

Example 123

Solve the Cauchy problem $y^{\prime}-y \cot x=\mathrm{e}^{x} \sin x, y\left(\frac{\pi}{2}\right)=0$.

First, we find the general solution of associated homogeneous LODE,

$$
y^{\prime}-y \cot x=0 \Rightarrow \frac{\mathrm{d} y}{\mathrm{~d} x}=y \cot x \Rightarrow \int \frac{\mathrm{d} y}{y}=\int \cot x \mathrm{~d} x+C \Rightarrow \ln |y|=\ln |\sin x|+C .
$$

The general solution has the form $\hat{y}=C \sin x$.

We continue with the variation of the integration constant $C=C(x)$.

$$
y=C(x) \sin x \quad \Rightarrow \quad y^{\prime}=C^{\prime}(x) \sin x+C(x) \cos x
$$


After the substitution into the original LODE, we get:

$C^{\prime}(x) \sin x+C(x) \cos x-C(x) \sin x \cot x=\mathrm{e}^{x} \sin x \Rightarrow C^{\prime}(x) \sin x=\mathrm{e}^{x} \sin x \Rightarrow C^{\prime}(x)=\mathrm{e}^{x}$.

Thus

$$
C(x)=\int \mathrm{e}^{x} \mathrm{~d} x=\mathrm{e}^{x}+C
$$

We substitute into estimated general solution

$$
y=\left(\mathrm{e}^{x}+C\right) \sin x
$$

We determine the value of the constant $C$ from the initial condition $y\left(\frac{\pi}{2}\right)=0$

$$
0=\left(\mathrm{e}^{\frac{\pi}{2}}+C\right) \sin \frac{\pi}{2} \Rightarrow C=-\mathrm{e}^{\frac{\pi}{2}} .
$$

The solution of Cauchy problem i.e. the particular solution of the original LODE is

$$
y=\left(\mathrm{e}^{x}-\mathrm{e}^{\frac{\pi}{2}}\right) \sin x
$$

Exercise 124

Solve the differential equations
a) $y^{\prime}-\frac{y}{x}=x^{2}$
b) $y^{\prime}+y \cos x=\sin x \cos x$

Exercise 125

Solve the Cauchy problems
a) $y^{\prime}-y \frac{\sin x}{\cos x}=\frac{1}{\cos x}, y(0)=0$
b) $x y^{\prime}-\frac{y}{x+1}=x, y(1)=0$

\subsection{Linear diff. equations of the second order with constant coefficients}

If we solve concrete practical problems which are modelled by means of ODE, quite often we see that single parameters (weight, density, frequency, etc.) in differential equations are constants. Such tasks form a huge subclass in the category of 2 nd order ODE.

\section{Definition}

Linear ordinary differential equation (LODE) of the second order with constant coefficients has the form

$$
a_{2} y^{\prime \prime}(x)+a_{1} y^{\prime}(x)+a_{0} y(x)=b(x),
$$

where $a_{2} \neq 0, a_{1}, a_{0}$ are real constants and the function $b(x)$ is called right-hand side of the equations.

Next

- if $b(x)=0$, we talk about homogeneous (shortened) LODE,

- je-li $b(x) \neq 0$, we talk about non-homogeneous (complete) LODE. 
Remark

$y^{\prime \prime}+3 y^{\prime}=2-x \ldots$ non-homogeneous LODE

$y^{\prime \prime}+3 y^{\prime}=0 \quad \ldots$ associated homogeneous LODE

\section{Definition}

We call a fundamental system of solutions of the equation $a_{2} y^{\prime \prime}+a_{1} y^{\prime}+a_{0} y=0$ the pair of functions $y_{1}=y_{1}(x), y_{2}=y_{2}(x)$, if $y_{1}, y_{2}$ are two non-trivial linearly independent solutions of the given equation.

The pair of functions $y_{1}, y_{2}$ defined on $I$ and with continuous derivatives of the first order on $I$ is linearly independent if the determinant of the following matrix

$$
W=\left|\begin{array}{ll}
y_{1} & y_{2} \\
y_{1}^{\prime} & y_{2}^{\prime}
\end{array}\right| \neq 0
$$

at some point $x \in I$.

\section{Definition}

The function $W$ is called Wronski determinant or Wronskian.

\section{Example 126}

Show that the functions $y_{1}=\mathrm{e}^{3 x}, y_{2}=x \mathrm{e}^{3 x}$ are linearly independent on $\mathbb{R}$.

We calculate the Wronskian:

$$
W=\left|\begin{array}{cc}
\mathrm{e}^{3 x} & x \mathrm{e}^{3 x} \\
3 \mathrm{e}^{3 x} & \mathrm{e}^{3 x}+3 x \mathrm{e}^{3 x}
\end{array}\right|=\mathrm{e}^{3 x}\left(\mathrm{e}^{3 x}+3 x \mathrm{e}^{3 x}\right)-3 x \mathrm{e}^{6 x}=\mathrm{e}^{6 x} .
$$

Because $\mathrm{e}^{6 x} \neq 0$ for $\forall x \in \mathbb{R}$, the functions $y_{1}=\mathrm{e}^{3 x}, y_{2}=x \mathrm{e}^{3 x}$ are linearly independent on $\mathbb{R}$.

\subsection{Characteristic equation}

Let us consider homogeneous LODE $a_{2} y^{\prime \prime}+a_{1} y^{\prime}+a_{0} y=0$. We show that all solutions can be found without integration and can be expressed by means of elementary functions.

Let us take a closer look at the homogeneous LODE of the first order with constant coefficients $1, a$.

$$
\begin{aligned}
1 y^{\prime}+a y & =0 \\
y^{\prime} & =-a y \\
\frac{1}{y} \mathrm{~d} y & =-a \mathrm{~d} x \\
\hat{y} & =C \mathrm{e}^{-a x}
\end{aligned}
$$

A particular solution of this equation is the function of the form $y=\mathrm{e}^{r x}$, where $r \in \mathbb{R}$. 
For the analogous situation, i.e. for homogeneous LODE of the second order, we look for the solution of the same form $y=\mathrm{e}^{r x}$ and determine a condition for $r$. We put the estimated solution $y=\mathrm{e}^{r x}$ and its first and second order derivatives, $y^{\prime}=r \mathrm{e}^{r x}, y^{\prime \prime}=r^{2} \mathrm{e}^{r x}$ into the equation $a_{2} y^{\prime \prime}+a_{1} y^{\prime}+a_{0} y=0$. We get

$$
\begin{aligned}
a_{2} y^{\prime \prime}+a_{1} y^{\prime}+a_{0} y & =0 \\
a_{2} r^{2} \mathrm{e}^{r x}+a_{1} r \mathrm{e}^{r x}+a_{0} \mathrm{e}^{r x} & =0 \\
\mathrm{e}^{r x}\left(a_{2} r^{2}+a_{1} r+a_{0}\right) & =0
\end{aligned}
$$

Because $\mathrm{e}^{r x} \neq 0$ for $\forall x \in \mathbb{R}$, it holds that $y=\mathrm{e}^{r x}$ is a solution if $r$ is a solution of the equation

$$
a_{2} r^{2}+a_{1} r+a_{0}=0
$$

called characteristic equation. It is a simple quadratic equation for the unknown $r$.

Characteristic equation can be simply obtained from original LODE. We just write $r^{2}$ for $y^{\prime \prime}, r$ for $y^{\prime}$ and 1 for $y$.

\section{Definition}

The equation

$$
a_{2} r^{2}+a_{1} r+a_{0}=0
$$

is called characteristic equation.

We distinguish the following three cases connected with the sign of the discriminant $D$ of the above quadratic equation:

- if $D>0$, then the characteristic equation has two real roots $r_{1}, r_{2} \in \mathbb{R}, r_{1} \neq r_{2}$, the fundamental system of solutions is $y_{1}=\mathrm{e}^{r_{1} x}, y_{2}=\mathrm{e}^{r_{2} x}$ and general solution takes the form

$$
\hat{y}=C_{1} \mathrm{e}^{r_{1} x}+C_{2} \mathrm{e}^{r_{2} x},
$$

where $C_{1}, C_{2} \in \mathbb{R}$,

- if $D=0$, then the characteristic equation has one double root $r$, the fundamental system of solutions is $y_{1}=\mathrm{e}^{r x}, y_{2}=x \mathrm{e}^{r x}$ and general solution takes the form

$$
\hat{y}=C_{1} \mathrm{e}^{r x}+C_{2} x \mathrm{e}^{r x},
$$

where $C_{1}, C_{2} \in \mathbb{R}$,

- if $D<0$, then the characteristic equation has no real roots, i.e. it has two complex conjugate roots $r_{1,2}=\alpha \pm \mathrm{i} \beta$, the fundamental system of solutions is $y_{1}=\mathrm{e}^{\alpha x} \cos \beta x$, $y_{2}=\mathrm{e}^{\alpha x} \sin \beta x$ and general solution takes the form

$$
\hat{y}=C_{1} \mathrm{e}^{\alpha x} \cos \beta x+C_{2} \mathrm{e}^{\alpha x} \sin \beta x,
$$

where $C_{1}, C_{2} \in \mathbb{R}$. 


\section{Example 127}

Solve the differential equations
a) $y^{\prime \prime}-5 y^{\prime}+6 y=0$
b) $y^{\prime \prime}-4 y^{\prime}+4 y=0$
c) $y^{\prime \prime}+2 y^{\prime}+5 y=0$

a)

$$
y^{\prime \prime}-5 y^{\prime}+6 y=0 \Rightarrow r^{2}-5 r+6=0 \Rightarrow r_{1}=2, r_{2}=3 \Rightarrow \hat{y}=C_{1} \mathrm{e}^{2 x}+C_{2} \mathrm{e}^{3 x}
$$

b)

$$
y^{\prime \prime}-4 y^{\prime}+4 y=0 \Rightarrow r^{2}-4 r+4=0 \Rightarrow r=2 \quad \Rightarrow \quad \hat{y}=C_{1} \mathrm{e}^{2 x}+C_{2} x \mathrm{e}^{2 x}
$$

c)

$$
\begin{gathered}
y^{\prime \prime}+2 y^{\prime}+5 y=0 \quad \Rightarrow \quad r^{2}+2 r+5=0 \quad \Rightarrow \\
r_{1,2}=\frac{-2 \pm \sqrt{4-20}}{2}=\frac{-2 \pm \sqrt{-16}}{2}=\frac{-2 \pm \mathrm{i} \sqrt{16}}{2}=-1 \pm 2 \mathrm{i} \quad \Rightarrow \\
\hat{y}=C_{1} \mathrm{e}^{-x} \cos 2 x+\mathrm{e}^{-x} \sin 2 x=\mathrm{e}^{-x}\left(C_{1} \cos 2 x+C_{2} \sin x\right)
\end{gathered}
$$

Note that in the third case, one can use either 2 or -2 as a value for $\beta$. There is no difference and we get the same solution (can you prove that), thus the positive value is preferred.

\section{Example 128}

The chain of the length equal to 3 meters slides off a smooth horizontal table. In the exact moment, when the chain begun to move from the table, a $30 \mathrm{~cm}$ long part of the chain hung from the table. How long will it take before the chain falls completely?

The movement of the chain is caused by gravitational field by means of the force acting on the part of a chain hanging from the table. Its magnitude is

$$
F=\frac{m}{l} y g,
$$

where $l$ is length of the chain, $m$ is weight of the chain and $y$ is length of the hanging part of the chain, gravitational acceleration $g=9.81 \mathrm{~m} \cdot \mathrm{s}^{-2}$.

Using the second Newton's law $F=m \ddot{y}$ and after some manipulations we get homogeneous second order ODE for $y=y(t)$

$$
\begin{aligned}
m \ddot{y}=\frac{m}{l} y g & \Rightarrow \ddot{y}-\frac{g}{l} y=0 \quad \Rightarrow \quad r^{2}-\frac{g}{l}=0 \Rightarrow r_{1,2}= \pm \sqrt{\frac{g}{l}} \\
& \Rightarrow \hat{y}=y=C_{1} \mathrm{e}^{\sqrt{\frac{g}{l}} t}+C_{2} \mathrm{e}^{-\sqrt{\frac{g}{l} t}} .
\end{aligned}
$$

We substitute the initial condition at the time $t=0, y(0)=0.3, \dot{y}(0)=0$,

$$
\begin{aligned}
y & =C_{1} \mathrm{e}^{\sqrt{\frac{g}{l}} t}+C_{2} \mathrm{e}^{-\sqrt{\frac{g}{l}} t}, \quad \dot{y}=C_{1} \sqrt{\frac{g}{l}} \mathrm{e}^{\sqrt{\frac{g}{l}} t}-C_{2} \sqrt{\frac{g}{l}} \mathrm{e}^{-\sqrt{\frac{g}{l}} t} \\
0.3 & =C_{1} \mathrm{e}^{0}+C_{2} \mathrm{e}^{0}, \quad 0=\sqrt{\frac{9.81}{3}} C_{1} \mathrm{e}^{0}-\sqrt{\frac{9.81}{3}} C_{2} \mathrm{e}^{0} \\
& \Rightarrow \quad C_{1}=C_{2} \Rightarrow 0.3=C_{1}+C_{1} \Rightarrow C_{1}=C_{2}=0.15
\end{aligned}
$$


and get the particular solution in the form

$$
y_{p}=y=0.15\left(\mathrm{e}^{\sqrt{\frac{8}{l}} t}+\mathrm{e}^{-\sqrt{\frac{8}{l}} t}\right) .
$$

The task is to determine the time, in which the chain falls off the table. Thus we need to express $t$ from the solution. We multiply the latter equation by $\frac{1}{0,15} \mathrm{e}^{\sqrt{\frac{g}{l}} t}$ and get quadratic equation for $\mathrm{e}^{\sqrt{\frac{8}{l}} t}$,

$$
\left(\mathrm{e}^{\sqrt{\frac{g}{T}} t}\right)^{2}-\frac{1}{0.15} \mathrm{e}^{\sqrt{\frac{8}{T}} t} y+1=0 \quad \Rightarrow \quad \mathrm{e}^{\sqrt{\frac{8}{T}} t}=\frac{1}{0.3} y \pm \sqrt{\frac{1}{4 \cdot 0.15^{2}} y^{2}-1}
$$

The time must be positive, thus the only $\mathrm{e}^{\sqrt{\frac{g}{T}} t}=\frac{1}{0,3} y+\sqrt{\frac{1}{4 \cdot 0.15^{2}} y^{2}-1}$ makes sense, we substitute $y=l$ and express $t$ :

$$
t=\sqrt{\frac{l}{g}} \ln \left(\frac{l}{0.3}+\sqrt{\frac{1}{4 \cdot 0.15^{2}} l^{2}-1}\right)=\sqrt{\frac{3}{9.81}} \ln \left(\frac{3}{0.3}+\sqrt{\frac{3^{2}}{4 \cdot 0.15^{2}}-1}\right)=1.66
$$

The chain falls off the table in 1.66 seconds.

Exercise 129

Solve the differential equations
a) $y^{\prime \prime}-3 y^{\prime}+2 y=0$
b) $y^{\prime \prime}+6 y^{\prime}+9 y=0$
c) $y^{\prime \prime}-4 y^{\prime}+5 y=0$

\subsection{Non-homogeneous second order LODE - solution}

Let us consider non-homogeneous LODE

$$
a_{2} y^{\prime \prime}+a_{1} y^{\prime}+a_{0} y=b(x), \quad b(x) \neq 0 .
$$

General solution of the non-homogeneous LODE takes the form:

$$
y(x)=\hat{y}(x)+v(x),
$$

where $\hat{y}(x)$ is a solution of the associated homogeneous LODE and $v(x)$ is the particular solution of the corresponding original non-homogeneous LODE connected with concrete right-hand side $b(x)$.

We know how to find the solution $\hat{y}$ of the associated homogeneous LODE. The particular solution $v(x)$ can be found using two main methods:

- Variation of constants - universal method, however with more complicated calculations

- Method of undetermined coefficients - method suitable only for some special righthand sides of non-homogeneous LODE, however the solution obtained with less effort

The next theorem represents superposition principle 


\section{Theorem}

Let the right-hand side $b(x)$ of the non-homogeneous LODE $a_{2} y^{\prime \prime}+a_{1} y^{\prime}+a_{0} y=b(x)$ can be decomposed into a sum

$$
b(x)=b_{1}(x)+b_{2}(x)+\cdots+b_{k}(x)
$$

and let $v_{j}(x)$ be particular solutions of the equation

$$
a_{2} y^{\prime \prime}+a_{1} y^{\prime}+a_{0} y=b_{j}(x), \quad j=1, \ldots, k,
$$

then

$$
v(x)=v_{1}(x)+v_{2}(x)+\cdots+v_{k}(x)
$$

is the particular solution of the original equation.

\subsection{Variation of constants}

The core of the method is the same as in the case of the 1st order LODE.

The next theorem characterizes Variation of constants for 2nd order LODE.

\section{Theorem}

General solution of the equation

$$
a_{2} y^{\prime \prime}+a_{1} y^{\prime}+a_{0} y=b(x)
$$

with constant coefficient $a_{2}, a_{1}, a_{0}$ one can express in the form

$$
y(x)=\hat{y}(x)+v(x)=\hat{y}(x)+y_{1}(x) \int \frac{W_{1}(x)}{W(x)} \mathrm{d} x+y_{2}(x) \int \frac{W_{2}(x)}{W(x)} \mathrm{d} x
$$

where $\hat{y}(x)=C_{1} y_{1}(x)+C_{2} y_{2}(x)$ is the general solution of associated homogeneous LODE, $W(x)$ is Wronskian of its fundamental system and $W_{1}(x), W_{2}(x)$ are determinants which come from Wronskian $W(x)$ by replacing its first respectively second column by the vector of right-hand side $\left(0, b(x) / a_{2}\right)$.

We solve the non-homogeneous 2nd order LODE with constant coefficients

$$
a_{2} y^{\prime \prime}+a_{1} y^{\prime}+a_{0} y=b(x)
$$

under the assumption that we know solutions of associated homogeneous LODE $\hat{y}=$ $C_{1} y_{1}(x)+C_{2} y_{2}(x)$.

Let us assume that general solution of non-homogeneous 2nd order LODE will have the same form as the solution of homogeneous LODE, however we replace integration constants $C_{1}, C_{2}$ by unknown functions $C_{1}(x), C_{2}(x)$. We make variation of constants. We seek the solution in the form

$$
y(x)=C_{1}(x) y_{1}(x)+C_{2}(x) y_{2}(x) .
$$


We calculate derivative of the estimated solution

$$
y^{\prime}=C_{1}^{\prime} y_{1}+C_{1} y_{1}^{\prime}+C_{2}^{\prime} y_{2}+C_{2} y_{2}^{\prime} .
$$

The choice of the new functions $C_{1}(x), C_{2}(x)$ allows us to set up a suitable additional condition,

$$
C_{1}^{\prime} y_{1}+C_{2}^{\prime} y_{2}=0
$$

Substituting the condition into the first derivative we get

$$
y^{\prime}=C_{1} y_{1}^{\prime}+C_{2} y_{2}^{\prime} \quad \text { and } \quad y^{\prime \prime}=C_{1}^{\prime} y_{1}^{\prime}+C_{1} y_{1}^{\prime \prime}+C_{2}^{\prime} y_{2}^{\prime}+C_{2} y_{2}^{\prime \prime}
$$

which we put into the original non-homogeneous LODE

$$
a_{2}(\underbrace{C_{1}^{\prime} y_{1}^{\prime}+C_{1} y_{1}^{\prime \prime}+C_{2}^{\prime} y_{2}^{\prime}+C_{2} y_{2}^{\prime \prime}}_{y^{\prime \prime}})+a_{1}(\underbrace{C_{1} y_{1}^{\prime}+C_{2} y_{2}^{\prime}}_{y^{\prime}})+a_{0}(\underbrace{C_{1} y_{1}+C_{2} y_{2}}_{y})=b(x)
$$

and get

$$
C_{1}\left(a_{2} y_{1}^{\prime \prime}+a_{1} y_{1}^{\prime}+a_{0} y_{1}\right)+C_{2}\left(a_{2} y_{2}^{\prime \prime}+a_{1} y_{2}^{\prime}+a_{0} y_{2}\right)+a_{2}\left(C_{1}^{\prime} y_{1}^{\prime}+C_{2}^{\prime} y_{2}^{\prime}\right)=b(x) .
$$

Because the functions $y_{1}, y_{2}$ are solutions of the associated homogeneous LODE, the expression in brackets must be null, and we get

$$
C_{1}^{\prime} y_{1}^{\prime}+C_{2}^{\prime} y_{2}^{\prime}=\frac{b(x)}{a_{2}}
$$

Thereby, we obtained second condition for derivatives of unknown functions $C_{1}(x), C_{2}(x)$ and we solve the following system of linear equations for the unknowns $C_{1}^{\prime}, C_{2}^{\prime}$,

$$
\begin{aligned}
& C_{1}^{\prime} y_{1}+C_{2}^{\prime} y_{2}=0, \\
& C_{1}^{\prime} y_{1}^{\prime}+C_{2}^{\prime} y_{2}^{\prime}=\frac{b(x)}{a_{2}} .
\end{aligned}
$$

The determinant of the system is the Wronskian of the functions $y_{1}, y_{2}$

$$
W=\left|\begin{array}{ll}
y_{1} & y_{2} \\
y_{1}^{\prime} & y_{2}^{\prime}
\end{array}\right|
$$

which is not null because both functions are linearly independent, they form fundamental system of solutions. The system has just one solution which can be found by the help of Cramer's rule

$$
C_{1}^{\prime}(x)=\frac{W_{1}(x)}{W(x)}, \quad C_{2}^{\prime}(x)=\frac{W_{2}(x)}{W(x)},
$$

where

$$
W_{1}=\left|\begin{array}{cc}
0 & y_{2} \\
\frac{b(x)}{a_{2}} & y_{2}^{\prime}
\end{array}\right| \quad \text { and } \quad W_{2}=\left|\begin{array}{cc}
y_{1} & 0 \\
y_{1}^{\prime} & \frac{b(x)}{a_{2}}
\end{array}\right|
$$

After integration we get

$$
C_{1}(x)=\int \frac{W_{1}(x)}{W(x)} \mathrm{d} x+K_{1}, \quad C_{2}(x)=\int \frac{W_{2}(x)}{W(x)} \mathrm{d} x+K_{2}
$$


where $K_{1}, K_{2} \in \mathbb{R}$. If we substitute into the estimated solution $y(x)=C_{1}(x) y_{1}(x)+$ $C_{2}(x) y_{2}(x)$ we get general solution of non-homogeneous LODE in the form

$$
y(x)=\hat{y}(x)+v(x)=\underbrace{K_{1} y_{1}(x)+K_{2} y_{2}(x)}_{\hat{y}(x)}+y_{1}(x) \underbrace{\int \frac{W_{1}(x)}{W(x)} \mathrm{d} x+y_{2}(x) \int \frac{W_{2}(x)}{W(x)} \mathrm{d} x}_{v(x)} .
$$

Finally, one can use the same letters $C$ for the integrations constants, thus

$$
y(x)=\hat{y}(x)+v(x)=\underbrace{C_{1} y_{1}(x)+C_{2} y_{2}(x)}_{\hat{y}(x)}+y_{1}(x) \underbrace{\int \frac{W_{1}(x)}{W(x)} \mathrm{d} x+y_{2}(x) \int \frac{W_{2}(x)}{W(x)} \mathrm{d} x}_{v(x)} .
$$

\section{Example 130}

Find the general solution of $\operatorname{LODE} y^{\prime \prime}+y=5 \mathrm{e}^{2 x}$.

We find the solution of the associated homogeneous LODE:

$$
y^{\prime \prime}+y=0 \Rightarrow r^{2}+1=0 \quad \Rightarrow \quad r_{1,2}= \pm \mathrm{i}
$$

The characteristic equation has two complex conjugate roots, whereas $\alpha=0, \beta=1$, we find the solution and make variation of constants:

$$
\hat{y}=C_{1} \cos x+C_{2} \sin x \Rightarrow y=C_{1}(x) \cos x+C_{2}(x) \sin x .
$$

For determining the unknown functions $C_{1}(x)$ and $C_{2}(x)$ we calculate determinants:

$$
\begin{gathered}
W(x)=\left|\begin{array}{rr}
\cos x & \sin x \\
-\sin x & \cos x
\end{array}\right|=\cos ^{2} x+\sin ^{2} x=1 \\
W_{1}(x)=\left|\begin{array}{cc}
0 & \sin x \\
5 \mathrm{e}^{2 x} & \cos x
\end{array}\right|=-5 \mathrm{e}^{2 x} \sin x, \quad W_{2}(x)=\left|\begin{array}{cc}
\cos x & 0 \\
-\sin x & 5 \mathrm{e}^{2 x}
\end{array}\right|=5 \mathrm{e}^{2 x} \cos x .
\end{gathered}
$$

Next, we calculate integration constants $C_{1}(x)$ and $C_{2}(x)$ :

$$
\begin{gathered}
C_{1}^{\prime}=\frac{W_{1}(x)}{W(x)}=-5 \mathrm{e}^{2 x} \sin x \quad \Rightarrow \quad C_{1}(x)=-5 \int \mathrm{e}^{2 x} \sin x \mathrm{~d} x=\mathrm{e}^{2 x}(\cos x-2 \sin x)+C_{1}, \\
C_{2}^{\prime}=\frac{W_{2}(x)}{W(x)}=5 \mathrm{e}^{2 x} \cos x \quad \Rightarrow \quad C_{2}(x)=5 \int \mathrm{e}^{2 x} \cos x \mathrm{~d} x=\mathrm{e}^{2 x}(2 \cos x+\sin x)+C_{2} .
\end{gathered}
$$

We substitute $C_{1}(x)$ and $C_{2}(x)$ into $y=C_{1}(x) \cos x+C_{2}(x) \sin x$ and get

$$
y=\left(\mathrm{e}^{2 x}(\cos x-2 \sin x)+C_{1}\right) \cos x+\left(\mathrm{e}^{2 x}(2 \cos x+\sin x)+C_{2}\right) \sin x .
$$

After some manipulations we get the general solution in the form

$$
y=\hat{y}(x)+v(x)=C_{1} \cos x+C_{2} \sin x+\mathrm{e}^{2 x} .
$$


Example 131

Find the general solution of LODE $y^{\prime \prime}+3 y^{\prime}+2 y=\frac{1}{1+\mathrm{e}^{x}}$.

We find the solution of associated homogeneous LODE:

$$
y^{\prime \prime}+3 y^{\prime}+2 y=0 \Rightarrow r^{2}+3 r+2=0 \quad \Rightarrow \quad r_{1}=-1, r_{2}=-2
$$

The characteristic eq. has two different real roots, we find the solution and make variation of constants:

$$
\hat{y}=C_{1} \mathrm{e}^{-x}+C_{2} \mathrm{e}^{-2 x} \Rightarrow y=C_{1}(x) \mathrm{e}^{-x}+C_{2}(x) \mathrm{e}^{-2 x} .
$$

For determining unknown functions $C_{1}(x)$ and $C_{2}(x)$ we calculate determinants

$$
\begin{gathered}
W(x)=\left|\begin{array}{cc}
\mathrm{e}^{-x} & \mathrm{e}^{-2 x} \\
-\mathrm{e}^{-x} & -2 \mathrm{e}^{-2 x}
\end{array}\right|=-2 \mathrm{e}^{-3 x}+\mathrm{e}^{-3 x}=-\mathrm{e}^{-3 x}, \\
W_{1}(x)=\left|\begin{array}{cc}
0 & \mathrm{e}^{-2 x} \\
\frac{1}{1+\mathrm{e}^{x}} & -2 \mathrm{e}^{-2 x}
\end{array}\right|=-\frac{\mathrm{e}^{-2 x}}{1+\mathrm{e}^{x}}, \quad W_{2}(x)=\left|\begin{array}{cc}
\mathrm{e}^{-x} & 0 \\
-\mathrm{e}^{-x} & \frac{1}{1+\mathrm{e}^{x}}
\end{array}\right|=\frac{\mathrm{e}^{-x}}{1+\mathrm{e}^{x}} .
\end{gathered}
$$

Next, we calculate integration constants $C_{1}(x)$ and $C_{2}(x)$ :

$$
\begin{gathered}
C_{1}^{\prime}=\frac{W_{1}(x)}{W(x)}=-\frac{\mathrm{e}^{-2 x}}{\left(1+\mathrm{e}^{x}\right)\left(-\mathrm{e}^{-3 x}\right)}=\frac{\mathrm{e}^{x}}{1+\mathrm{e}^{x}} \Rightarrow C_{1}(x)=\int \frac{\mathrm{e}^{x}}{1+\mathrm{e}^{x}} \mathrm{~d} x=\ln \left|1+\mathrm{e}^{x}\right|+C_{1}, \\
C_{2}^{\prime}=\frac{W_{2}(x)}{W(x)}=\frac{\mathrm{e}^{-x}}{\left(1+\mathrm{e}^{x}\right)\left(-\mathrm{e}^{-3 x}\right)}=-\frac{\mathrm{e}^{2 x}}{1+\mathrm{e}^{x}} \Rightarrow C_{2}(x)=-\int \frac{\mathrm{e}^{2 x}}{1+\mathrm{e}^{x}} \mathrm{~d} x=\ln \left|1+\mathrm{e}^{x}\right|-\mathrm{e}^{x}+C_{2} .
\end{gathered}
$$

We substitute $C_{1}(x)$ and $C_{2}(x)$ into $y=C_{1}(x) \mathrm{e}^{-x}+C_{2}(x) \mathrm{e}^{-2 x}$ and get

$$
y=\left(\ln \left|1+\mathrm{e}^{x}\right|+C_{1}\right) \mathrm{e}^{-x}+\left(\ln \left|1+\mathrm{e}^{x}\right|-\mathrm{e}^{x}+C_{2}\right) \mathrm{e}^{-2 x} .
$$

After some manipulations we get the general solution in the form

$$
y=\hat{y}(x)+v(x)=C_{1} \mathrm{e}^{-x}+C_{2} \mathrm{e}^{-2 x}+\mathrm{e}^{-x} \ln \left|1+\mathrm{e}^{x}\right|+\mathrm{e}^{-2 x}\left(\ln \left|1+\mathrm{e}^{x}\right|-\mathrm{e}^{x}\right) .
$$

\section{Exercise 132}

Solve the differential equation
a) $y^{\prime \prime}-5 y^{\prime}+6 y=6 x^{2}-10 x-4$
b) $y^{\prime \prime}-4 y^{\prime}=4 \sin x-\cos x$

\subsection{Undetermined coefficients}

The method is based on an estimate of the form of particular solution with respect to righthand side of LODE. Only special right-hand sides are allowed - polynomials, exponential functions or linear combination of sine and cosine functions. The form of the special righthand side is specified by the next theorem. 


\section{Method of undermined coefficients for 2 nd order LODE}

Theorem

Let the right-hand side of LODE with constant coefficients have the form

$$
b(x)=\mathrm{e}^{\lambda x}\left(p_{m}(x) \cos \omega x+q_{n}(x) \sin \omega x\right),
$$

where $p_{m}(x), q_{n}(x)$ are polynomials of degrees $m$ or $n$ and $\lambda, \omega \in \mathbb{R}$. If the numbers $\bar{r}=\lambda \pm \mathrm{i} \omega$ are $k$-fold roots of the characteristic equation, then we take the particular solution of the form

$$
v(x)=x^{k} \mathrm{e}^{\lambda x}\left(P_{M}(x) \cos \omega x+Q_{M}(x) \sin \omega x\right),
$$

where $M=\max \{m, n\}, P_{M}(x)$ and $Q_{M}(x)$ are polynomial of degree $M$ with undetermined coefficients.

We determine the coefficients of the polynomials $P_{M}(x)$ and $Q_{M}(x)$ using comparative method after substitution of the particular solution into the original LODE.

- Remark

- special right-hand sides: $\sin x, x \mathrm{e}^{-x} \cos (2 x), 3, \mathrm{e}^{-3 x}$

- not special right-hand sides: $\sin x \cos (3 x), \ln x, \mathrm{e}^{x^{2}+2}$

We estimate the form of the particular solution with help of the following table:

$p_{m}(x), P_{m}(x), Q_{m}(x)$ are polynomials of degree $m$,

$A_{m} x^{m}+\cdots+A_{1} x+A_{0}$ is a polynomial with undetermined coefficients,

$A_{m}, \ldots, A_{1}, A_{0} \in \mathbb{R}$

\begin{tabular}{lll}
\hline$b(x)$ & root of char. eq. & $v(x)$ \\
\hline$p_{m}(x)$ & $\bar{r}=0, k$-fold & $x^{k} P_{m}(x)$ \\
& $\bar{r}=0 \neq r$ & $P_{m}(x)$ \\
\hline & $\bar{r}=\lambda, k$-fold & $A x^{k} \mathrm{e}^{\lambda x}$ \\
$\mathrm{e}^{\lambda x}$ & $\bar{r}=\lambda \neq r$ & $A \mathrm{e}^{\lambda x}$ \\
\hline $\sin \omega x, \cos \omega x$ & $\bar{r}= \pm \mathrm{i} \omega$ & $x(A \cos \omega x+B \sin \omega x)$ \\
$\sin \omega x, \cos \omega x$ & $\bar{r}= \pm \mathrm{i} \omega \neq r$ & $A \cos \omega x+B \sin \omega x$ \\
\hline $\mathrm{e}^{\lambda x} p_{m}(x) \sin \omega x$ & $\bar{r}=\lambda \pm \mathrm{i} \omega$ & $x \mathrm{e}^{\lambda x}\left(P_{m}(x) \cos \omega x+Q_{m}(x) \sin \omega x\right)$ \\
$\mathrm{e}^{\lambda x} p_{m}(x) \cos \omega x$ & $\bar{r}=\lambda \pm \mathrm{i} \omega$ & $x \mathrm{e}^{\lambda x}\left(P_{m}(x) \cos \omega x+Q_{m}(x) \sin \omega x\right)$ \\
$\mathrm{e}^{\lambda x} p_{m}(x) \sin \omega x$ & $\bar{r}=\lambda \pm \mathrm{i} \omega \neq r$ & $\mathrm{e}^{\lambda x}\left(P_{m}(x) \cos \omega x+Q_{m}(x) \sin \omega x\right)$ \\
$\mathrm{e}^{\lambda x} p_{m}(x) \cos \omega x$ & $\bar{r}=\lambda \pm \mathrm{i} \omega \neq r$ & $\mathrm{e}^{\lambda x}\left(P_{m}(x) \cos \omega x+Q_{m}(x) \sin \omega x\right)$ \\
\hline
\end{tabular}


Note that we denote by letter $r$ the roots of characteristic polynomial. We compare the roots of characteristic polynomial with the number $\bar{r}$ determined by the right-hand side $b(x)$ of LODE.

\section{Example 133}

Try to estimate the form of a particular solution of the differential equation $y^{\prime \prime}+5 y^{\prime}=b(x)$ :
a) $b(x)=5 x^{2}$
c) $b(x)=3 \mathrm{e}^{5 x}$
e) $b(x)=\mathrm{e}^{-x} \cos 2 x$
b) $b(x)=\sin (5 x)$
d) $b(x)=3 x \mathrm{e}^{-5 x}$
f) $b(x)=\mathrm{e}^{x}+2$

We find the roots of the characteristic equation:

$$
y^{\prime \prime}+5 y^{\prime}=0 \quad \Rightarrow \quad r^{2}+5 r=0 \quad \Rightarrow \quad r_{1}=0, r_{2}=-5
$$

a)

$$
b(x)=5 x^{2} \quad \Rightarrow \quad \lambda=0, \omega=0 \quad \Rightarrow \quad \bar{r}=0,
$$

but $\bar{r}=0$ is simple root of the characteristic equation, thus we need to add factor $x$ to the estimated form of the particular solution, which is a polynomial of 2 nd degree with undetermined coefficients

$$
v(x)=x\left(A x^{2}+B x+C\right)
$$

b)

$$
b(x)=\sin 5 x \quad \Rightarrow \quad \lambda=0, \omega=5 \quad \Rightarrow \quad \bar{r}= \pm \mathrm{i} 5,
$$

but neither $\bar{r}=\mathrm{i} 5$ nor $\bar{r}=-\mathrm{i} 5$ are roots of the characteristic equation, thus the particular solution takes the form

$$
v(x)=A \cos 5 x+B \sin 5 x
$$

c)

$$
b(x)=3 \mathrm{e}^{5 x} \Rightarrow \lambda=5, \omega=0 \Rightarrow \bar{r}=5,
$$

but $\bar{r}=5$ is not the root of the characteristic equation,

$$
v(x)=A \mathrm{e}^{5 x}
$$

d)

$$
b(x)=3 x \mathrm{e}^{-5 x} \Rightarrow \lambda=-5, \omega=0 \quad \Rightarrow \quad \bar{r}=-5,
$$

but $\bar{r}=-5$ is simple root of the characteristic equation, we need to add factor $x$, thus

$$
v(x)=x(A x+B) \mathrm{e}^{-5 x}
$$

e)

$$
b(x)=\mathrm{e}^{-x} \cos 2 x \quad \Rightarrow \quad \lambda=-1, \omega=2 \quad \Rightarrow \quad \bar{r}=-1 \pm \mathrm{i} 2,
$$

but $\bar{r}$ are not the roots of the characteristic equation,

$$
v(x)=\mathrm{e}^{-x}(A \cos 2 x+B \sin 2 x)
$$


f)

$$
b(x)=\mathrm{e}^{x}+2
$$

it is not special right-hand side because there is addition between exponential and constant. Right-hand side can be considered as addition of two functions, each of which has the form of a special right-hand side, one can use superposition principle

$$
v(x)=v_{1}(x)+v_{2}(x),
$$

where $v_{1}(x)$ relates $b_{1}(x)=\mathrm{e}^{x}$ and $v_{2}(x)$ relates $b_{2}(x)=2$

$v_{1}(x)=A \mathrm{e}^{x}, \bar{r}=1$ is not the root of the characteristic equation

$v_{2}(x)=B x, \bar{r}=0$ is simple root of the characteristic equation

$$
v(x)=A \mathrm{e}^{x}+B x
$$

To find unknown coefficients we need to substitute estimated particular solution into original non-homogeneous LODE.

Example 134

Solve LODE $y^{\prime \prime}+4 y=-2$.

We find the solution of associated homogeneous equation:

$$
\begin{aligned}
y^{\prime \prime}+4 y & =0, \\
r^{2}+4 & =0, \\
r_{1,2} & = \pm 2 \mathrm{i} .
\end{aligned}
$$

The characteristic equation has two complex conjugate roots, whereas $\alpha=0, \beta=2$, thus the solution takes the form:

$$
\hat{y}=C_{1} \cos 2 x+C_{2} \sin 2 x .
$$

$b(x)=-2$ - special right-hand side $\Rightarrow$ we use the method of undermined coefficients, $\lambda=0, \omega=0, \Rightarrow \bar{r}=\lambda \pm \mathrm{i} \omega=0$, but $\bar{r}=0$ is not the root of the characteristic equation

Particular solution takes the form: $v(x)=A$.

We calculate the first and second order derivatives: $v^{\prime}=0, v^{\prime \prime}=0$, and substitute them into $y^{\prime \prime}+4 y=-2$ and get:

$$
0+4 A=-2 \Rightarrow A=-\frac{1}{2} .
$$

Particular solution:

$$
v=-\frac{1}{2}
$$

General solution $y=\hat{y}(x)+v(x)$ :

$$
y=\hat{y}(x)+v(x)=C_{1} \cos 2 x+C_{2} \sin 2 x-\frac{1}{2} .
$$


Example 135

Solve LODE $y^{\prime \prime}-10 y^{\prime}+24 y=(3 x-1) \mathrm{e}^{3 x}$.

We find the solution of associated homogeneous equation:

$$
y^{\prime \prime}-10 y^{\prime}+24 y=0 \quad \Rightarrow \quad r^{2}-10 r+24=0 \quad \Rightarrow \quad r_{1}=4, r_{2}=6,
$$

thus the solution takes the form

$$
\hat{y}=C_{1} \mathrm{e}^{4 x}+C_{2} \mathrm{e}^{6 x}
$$

$b(x)=(3 x-1) \mathrm{e}^{3 x}$ - special right-hand side $\Rightarrow$ we use the method of undetermined coefficients, $\lambda=3, \omega=0 \Rightarrow \bar{r}=\lambda \pm \mathrm{i} \omega=3$, but $\bar{r}=3$ is not the root of the characteristic equation

Particular solution: $v(x)=(A x+B) \mathrm{e}^{3 x}$.

Derivatives:

$$
v^{\prime}=A \mathrm{e}^{3 x}+3(A x+B) \mathrm{e}^{3 x}, \quad v^{\prime \prime}=6 A \mathrm{e}^{3 x}+9 \mathrm{e}^{3 x}(A x+B) .
$$

We substitute the estimated particular solution and its derivatives into original LODE

$$
\underbrace{6 A \mathrm{e}^{3 x}+9 \mathrm{e}^{3 x}(A x+B)}_{v^{\prime \prime}}-10 \underbrace{\left(A \mathrm{e}^{3 x}+3(A x+B) \mathrm{e}^{3 x}\right)}_{v^{\prime}}+24 \underbrace{(A x+B) \mathrm{e}^{3 x}}_{v(x)}=(3 x-1) \mathrm{e}^{3 x}
$$

and after some manipulations we get

$$
3 A x \mathrm{e}^{3 x}+(-4 A+3 B) \mathrm{e}^{3 x}=3 x \mathrm{e}^{3 x}-\mathrm{e}^{3 x} .
$$

We compare coefficients of the expressions $x \mathrm{e}^{3 x}$ and $\mathrm{e}^{3 x}$ on both sides of the equation

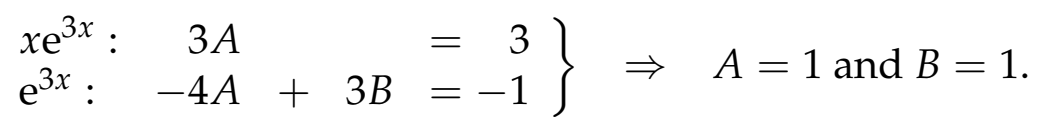

Particular solution

$$
v(x)=(x+1) \mathrm{e}^{3 x}
$$

General solution

$$
y=\hat{y}(x)+v(x)=C_{1} \mathrm{e}^{4 x}+C_{2} \mathrm{e}^{6 x}+(x+1) \mathrm{e}^{3 x} .
$$

\section{Example 136}

Solve LODE $y^{\prime \prime}-2 y^{\prime}+2 y=\mathrm{e}^{x} \sin x$.

We find the solution of associated homogeneous equation:

$$
y^{\prime \prime}-2 y^{\prime}+2 y=0 \quad \Rightarrow \quad r^{2}-2 r+2=0 \quad \Rightarrow \quad r_{1,2}=1 \pm \mathrm{i},
$$

thus the solution takes the form

$$
\hat{y}(x)=C_{1} \mathrm{e}^{x} \cos x+C_{2} \mathrm{e}^{x} \sin x .
$$


$b(x)=\mathrm{e}^{x} \sin x$-special right-hand side $\Rightarrow$ we use the method of undetermined coefficients, $\lambda=1, \omega=1 \Rightarrow \bar{r}=\lambda \pm \mathrm{i} \omega=1 \pm \mathrm{i}$, but $\bar{r}=1 \pm \mathrm{i}$ are simple roots of the characteristic equation

Particular solution: $v(x)=x \mathrm{e}^{x}(A \sin x+B \cos x)$.

Derivatives

$$
\begin{aligned}
v^{\prime} & =\mathrm{e}^{x}(A \sin x+B \cos x)+x \mathrm{e}^{x}(A \sin x+B \cos x)+x \mathrm{e}^{x}(A \cos x-B \sin x), \\
v^{\prime \prime} & =2 \mathrm{e}^{x}((A+B+B x) \cos x-(A-B+A x) \sin x) .
\end{aligned}
$$

We substitute the estimated solution and its derivatives into original LODE

$$
\begin{array}{r}
2 \mathrm{e}^{x}((A+B+B x) \cos x-(A-B+A x) \sin x)-2 \mathrm{e}^{x}(A \sin x+B \cos x+x(A \sin x \\
+B \cos x+A \cos x-B \sin x))+2 x \mathrm{e}^{x}(A \sin x+B \cos x)=\mathrm{e}^{x} \sin x,
\end{array}
$$

and after some manipulations we get

$$
2 B \cos x-2 A \sin x=\sin x .
$$

We compare coefficients of the expressions $\cos x$ and $\sin x$ on both sides of the equation

$$
\left.\begin{array}{rrr}
\cos x: & 2 B & =0 \\
\sin x:-2 A & & =1
\end{array}\right\} \Rightarrow A=-\frac{1}{2} \text { and } B=0 .
$$

Particular solution

$$
v(x)=-\frac{1}{2} x \mathrm{e}^{x} \sin x
$$

General solution

$$
y=\hat{y}(x)+v(x)=C_{1} \mathrm{e}^{x} \cos x+C_{2} \mathrm{e}^{x} \sin x-\frac{1}{2} x \mathrm{e}^{x} \sin x .
$$

\section{Example 137}

Solve LODE $y^{\prime \prime}-2 y^{\prime}+y=2 x^{2}+\mathrm{e}^{x}-2$.

We find the solution of associated homogeneous equation:

$$
y^{\prime \prime}-2 y^{\prime}+y=0 \quad \Rightarrow \quad r^{2}-2 r+1=0 \quad \Rightarrow \quad r_{1,2}=r=1 \quad \Rightarrow \quad \hat{y}=C_{1} \mathrm{e}^{x}+C_{2} x \mathrm{e}^{x} .
$$

$b(x)=2 x^{2}+\mathrm{e}^{x}-2$ - is not special right-hand side, but one can use superposition principle and decompose the right-hand side as

$$
b(x)=2 x^{2}-2+\mathrm{e}^{x}=b_{1}(x)+b_{2}(x), \text { where } b_{1}(x)=2 x^{2}-2, b_{2}(x)=\mathrm{e}^{x}
$$

Firstly, we deal with the special right-hand side represented by $b_{1}(x)$ :

$$
b_{1}(x)=2 x^{2}-2 \quad \Rightarrow \quad \bar{r}_{1}=0 \quad \text { (not root) }
$$

estimated solution: $v_{1}(x)=A x^{2}+B x+C$

derivatives:

$$
v_{1}^{\prime}=2 A x+B, \quad v_{1}^{\prime \prime}=2 A
$$

estimated solution we put in $y^{\prime \prime}-2 y^{\prime}+y=2 x^{2}-2$

$$
2 A-2(2 A x+B)+A x^{2}+B x+C=2 x^{2}-2
$$

comparing of coefficients: 


$$
\left.\begin{array}{rrr}
x^{2}: & A=2 \\
x^{1}: & -4 A+B=0 \\
x^{0}: & 2 A-2 B+C=-2
\end{array}\right\} \Rightarrow A=2, B=8 \text { a } C=10 .
$$

particular solution:

$$
v_{1}(x)=2 x^{2}+8 x+10
$$

Secondly, we deal with the special right-hand side $b_{2}(x)$ :

$$
b_{2}(x)=\mathrm{e}^{x} \quad \Rightarrow \quad \bar{r}_{2}=1 \quad \text { (double root) }
$$

estimated solution: $v_{2}(x)=A x^{2} \mathrm{e}^{x}$

derivatives:

$$
v_{2}^{\prime}=2 A x \mathrm{e}^{x}+A x^{2} \mathrm{e}^{x}, \quad v_{2}^{\prime \prime}=2 A \mathrm{e}^{x}+4 A x \mathrm{e}^{x}+A x^{2} \mathrm{e}^{x} .
$$

estimated sol. we put in $y^{\prime \prime}-2 y^{\prime}+y=\mathrm{e}^{x}$

$$
\begin{aligned}
& 2 A \mathrm{e}^{x}+4 A x \mathrm{e}^{x}+A x^{2} \mathrm{e}^{x}-2\left(2 A x \mathrm{e}^{x}+A x^{2} \mathrm{e}^{x}\right) \\
& \quad+A x^{2} \mathrm{e}^{x}=\mathrm{e}^{x}
\end{aligned}
$$

after some manipulations:

$$
2 A=1 \Rightarrow A=\frac{1}{2}
$$

particular solution:

$$
v_{2}(x)=\frac{1}{2} x^{2} \mathrm{e}^{x}
$$

General solution of LODE $y^{\prime \prime}-2 y^{\prime}+y=2 x^{2}+\mathrm{e}^{x}-2$ has the form

$$
y=\hat{y}(x)+v_{1}(x)+v_{2}(x)=C_{1} \mathrm{e}^{x}+C_{2} x \mathrm{e}^{x}+2 x^{2}+8 x+10+\frac{1}{2} x^{2} \mathrm{e}^{x} .
$$

\section{Exercise 138}

Solve LODE
a) $y^{\prime \prime}-2 y^{\prime}=x^{3}+2 x$
e) $y^{\prime \prime}-2 y^{\prime}+10 y=12 \mathrm{e}^{x} \cos 3 x$
b) $y^{\prime \prime}-3 y^{\prime}-4 y=3 \mathrm{e}^{2 x}$
f) $y^{\prime \prime}-5 y^{\prime}+4 y=\mathrm{e}^{x} \sin x$
c) $y^{\prime \prime}+y^{\prime}-2 y=2 x$
g) $y^{\prime \prime}-3 y^{\prime}-10 y=2(7 x+1) \mathrm{e}^{5 x}$
d) $y^{\prime \prime}+2 y^{\prime}=8 \cos 4 x$
h) $y^{\prime \prime}-5 y^{\prime}+6 y=6 x^{2}-10 x-4$

\section{Exercise 139}

Solve LODE $y^{\prime \prime}-4 y^{\prime}=4 \sin x-\cos x$.

\section{Exercise 140}

Solve Cauchy problem $y^{\prime \prime}+9 y=8 \cos x, \quad y\left(\frac{\pi}{3}\right)=0, y^{\prime}\left(\frac{\pi}{3}\right)=-\frac{\sqrt{3}}{2}$. 
Name: Workbook for Mathematics II

Department, Institute: Faculty of Civil Engineering, Department of Mathematics Authors: Jana Volná, Petr Volný

Place, year of publishing: Ostrava, 2021, 1st Edition

Number of Pages: 117

Published: VSB - Technical University of Ostrava 